\title{
On the Use of Fuzzy Logic to Control Paralleled DC-DC Converters
}

\author{
Bogdan Tomescu
}

Dissertation submitted to the Faculty of the Virginia Polytechnic Institute and State University in partial fulfillment of the requirements for the degree of

\author{
Doctor of Philosophy \\ in \\ Electrical and Computer Engineering \\ Hugh. F. VanLandingham, Chair \\ Dushan Boroyevich \\ William Baumann \\ Dusan Teodorovic \\ Harry H. Robertshaw
}

October 24, 2001

Blacksburg, Virginia

Keywords: DC-DC Converters, Fuzzy Logic Control, Master-Slave Structure, Parallel Converters, Lyapunov Stability, Soft Computing

Copyright 2001, Bogdan Tomescu 


\title{
On the Use of Fuzzy Logic to Control Paralleled DC-DC Converters
}

\author{
Bogdan Tomescu
}

\section{(ABSTRACT)}

The objective of the thesis is to introduce a new fuzzy logic control application, develop the associated mathematical theory and prove the concept and its advantages through comparative simulation with existing, classical, methods. A stable fuzzy logic controller for the master-slave current sharing loop of a paralleled DC-DC system is presented that exhibits a considerably improved large signal performance over the presently employed, small signal designed compensators, both in terms of system response and control effort. Because of high system complexity, the present small signal designs are unable to give a good response for large load changes and line transients. Fuzzy logic, by dealing naturally with nonlinearities, offers a superior controller type, for this type of applications. The design uses a PID expert to derive the fuzzy inference rules, and simulation results show a good parameter insensitive transient response over a wide range load-step responses, e.g., from $25 \%$ to $75 \%$ of the nominal load. Current sharing control is formulated as a tracking problem and stability is ensured through adaptation or supervisory control on a Lyapunov trajectory. The technique benefits also from the heuristic approach to the problem that overcomes the complexity in modeling such systems and, hence, offers a practical engineering tool, amenable to both analog and digital implementations. 


\section{Dedication}

This thesis is dedicated to my family: my parents, Marin and Maria, my grandparents, my uncle and aunt Milu and Tanți and all other close ones, present and past, whose unconditional love made my journey possible. 


\section{ACKNOWLEDGEMENTS}

I would first like to thank my family and friends for their warm support and patience during the research and writing of this thesis and throughout my career path. This includes all my friends, school and work colleagues and managers, both here in the US as well as from my early years in Europe, who encouraged my work and made valuable suggestions; their friendship is unforgettable.

Next, I would like to especially thank my teachers, exam committee members and peers who inspired and guided me.

My advisor, Prof. Hugh Foch VanLandingham, whose educational wisdom and understanding was my strongest support for this dissertation. He has shown me, once more, that management, teaching, research and fun can be all part of a good and balanced life.

Prof. Fred C. Lee and associated CPES staff, for allowing me the exceptional opportunity of starting research and study at Virginia Tech - VPEC. In this regard, working also with Prof. Dushan Boroyevich significantly broadened my research interests.

Prof. Slobodan Cuk, Prof. John Choma Jr., Mr. Robert Sauer, PE and Mrs. Loretta Estes for encouraging me in pursuing a doctoral program and their overall, kind and strong, support.

Physicist Teodor Ghicadia, who practically parented me into technical research.

Prof. Ioan Eftimie Teodorescu, at Polytechnic Institute of Bucharest, who taught me electronics with one of the highest levels of ethics, professionalism and warmth that I have ever known. Part of the same school and associated research teams, I would like to respectfully thank Prof. Gheorghe Hortopan, Dr. Dan Micu, Mr. Ovidiu Bizim, Prof. Serban Birca Galateanu, Dr. Alexandru Ivascu, Prof. Eugenia Stoenescu and Mrs. Maria Staicu, for their substantial help.

Special appreciation to Mariana Crivelescu for her care and understanding.

Finally, I thank all my college and earlier school teachers and educators who ultimately gave me this opportunity. 


\section{TABLE OF CONTENTS}

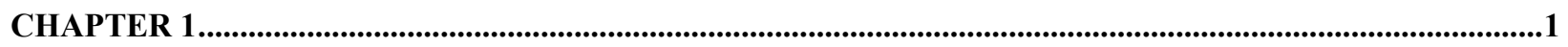

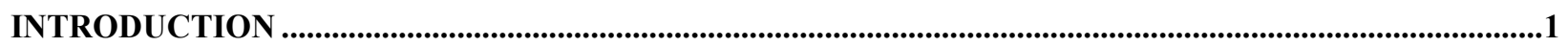

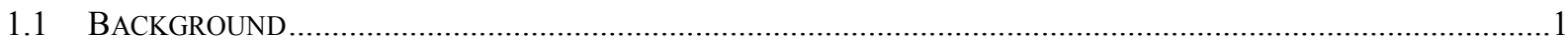

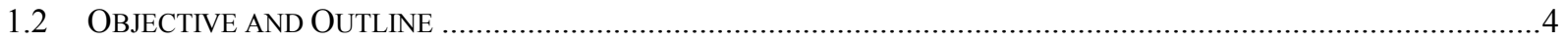

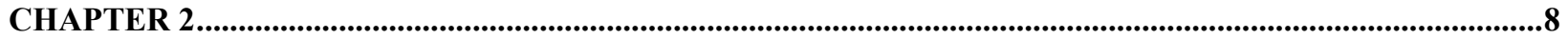

LARGE SIGNAL, ROBUSTNESS AND DISTURBANCE REJECTION ORIENTED MODELING FOR

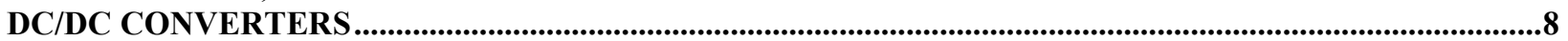

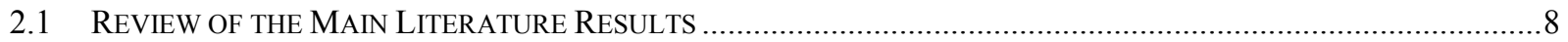

2.2 THE DC/DC CONVERTER AS A REGULATOR UNDER LINE AND LOAD DISTURBANCES ..................................12

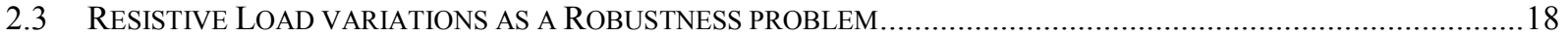

2.3.1 A Clarification for the Existing Small Signal Models ...................................................................18

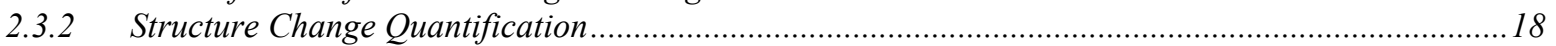

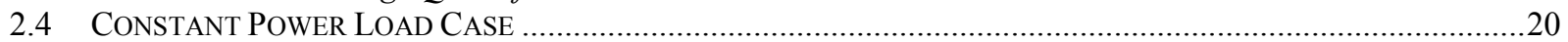

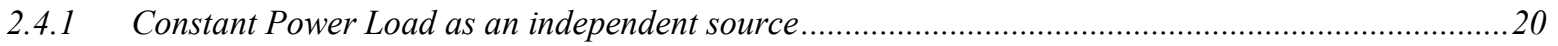

2.4.2 Constant Power load as an equivalent negative resistance ….....................................................21

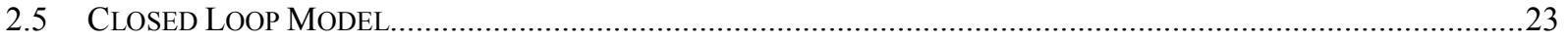

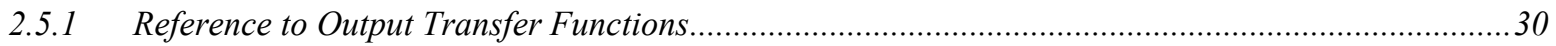

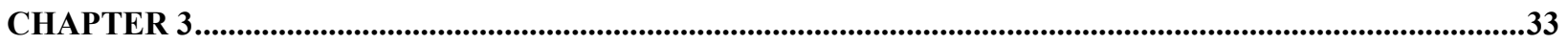

MODERN CONTROL SYSTEMS MODELING OF PARALLELED CONVERTER SYSTEMS ....................33

3.1 The Master - Slave Current Sharing Control as A Tracking Problem.............................................33

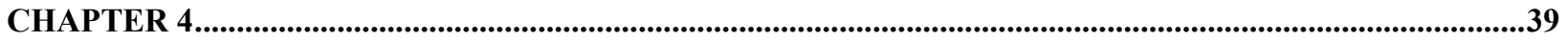

LYAPUNOV STABILITY ANALYSIS FOR DC/DC REGULATORS ….....................................................39

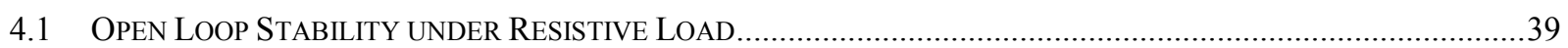

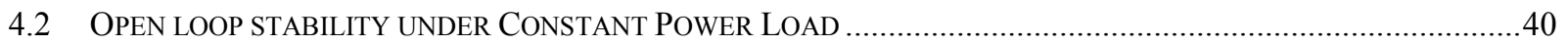

4.3 USE OF A LYAPUNOV APPROACH FOR INDIVIDUAL MODULE FEEDBACK DESIGN .........................................41

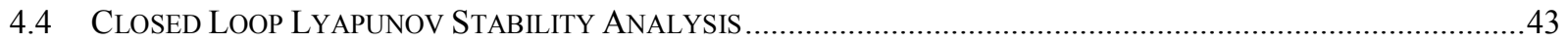

4.5 LYAPUNOV STABILITY FOR TWO IDENTICAL PARALLEL CONVERTERS ........................................................46

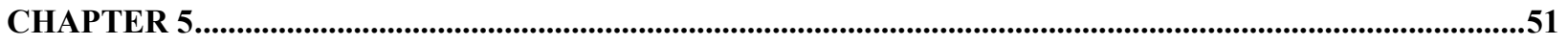

FUZZY LOGIC CONTROL OF PARALLELED DC/DC CONVERTER MODULES.........................................51

5.1 FUZZY LOGIC CONTROLLER DESIGN USING A PID COMPENSATOR AS EXPERT ...........................................51

5.1.1 Classical versus Fuzzy Logic Control for Active Current Sharing ................................................55

5.2 ON A LYAPUNOV STABLE FUZZY LOGIC CONTROL …….....................................................................59

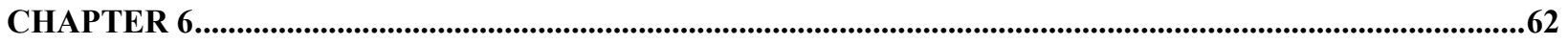

SIMULATED RESULTS OF FUZZY LOGIC CONTROLLED PARALLELED DC/DC CONVERTERS ...62

6.1 INDIVIDUAL CONVERTER MODULE DESIGN ANALYSIS .....................................................................62

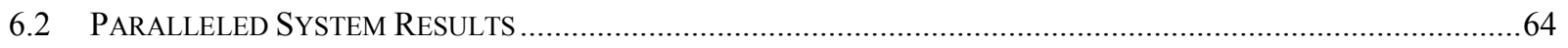

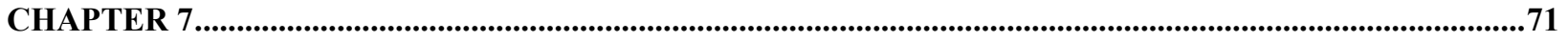




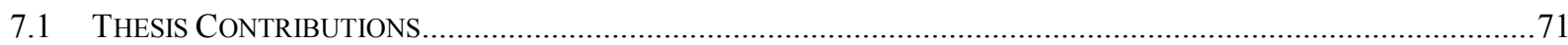

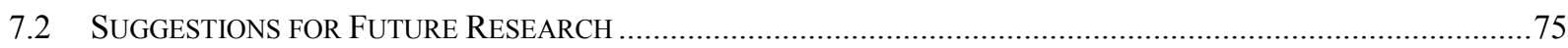

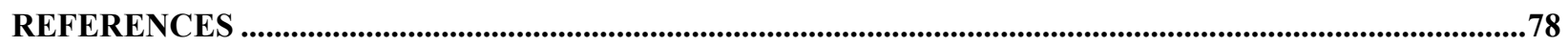

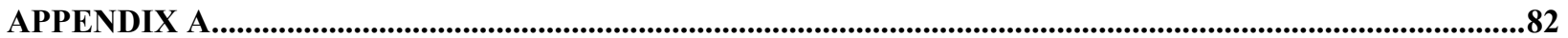

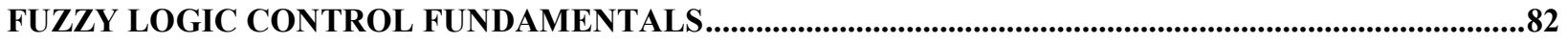

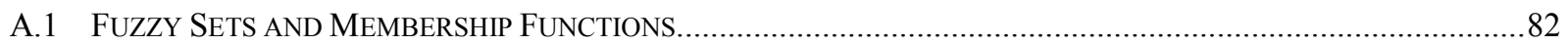

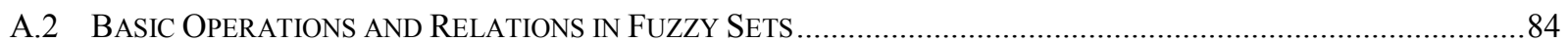

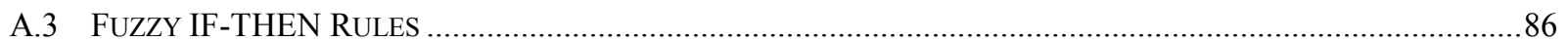

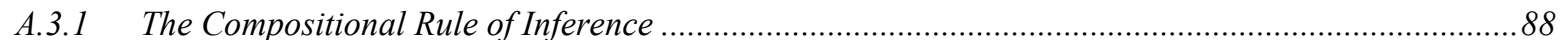

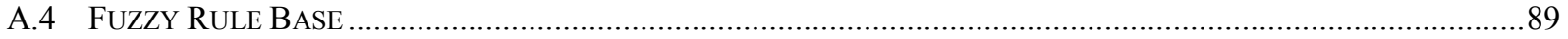

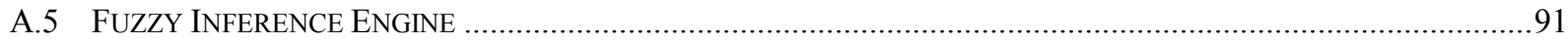

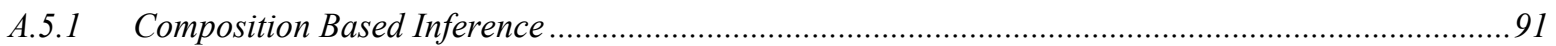

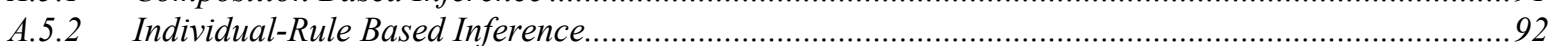

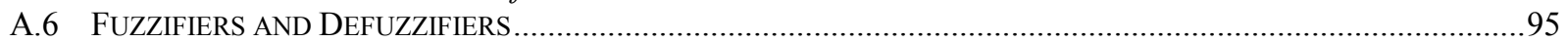

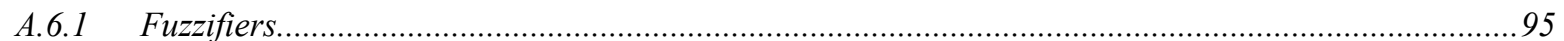

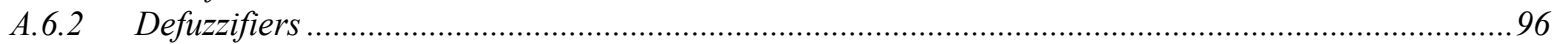

A.7 FUZZY SYSTEMS AS NONLINEAR MAPPINGS - FUZZY CONTROLLER STRUCTURE.........................................99

A.7.1 Fuzzy Systems with Center Average Defuzzifier ......................................................................

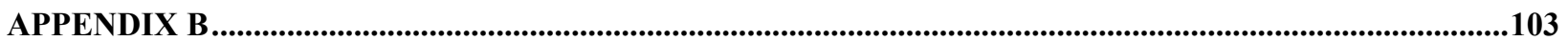

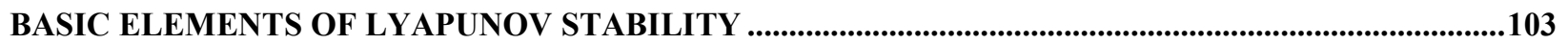

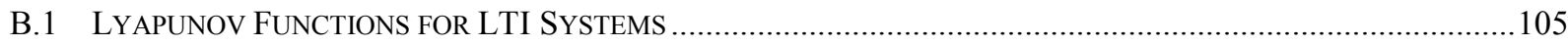

B.2 USE OF LyAPUNOV'S DiRECT METHOD IN FEEDBACK DESIGN ................................................................ 107

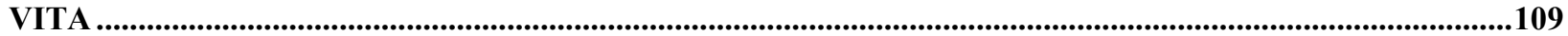




\section{LIST OF FIGURES}

FIGURE 2.1-1 - BOOST CONVERTER IN CCM USING THE RESISTIVE LOAD TERMINATED MODEL.................................9

FIGURE 2.2-1 - BOOST CONVERTER IN CCM USING THE UNTERMINATED MODEL ....................................................

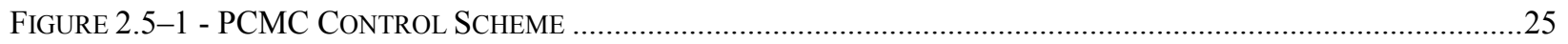

FIGURE 2.5-2 - PCMC DC/DC REGULATOR CONTROL BLOCK DIAGRAM ..........................................................26

FIGURE 2.5-3 - SMALl SIGNAL DIAGRAM OF A DC/DC CONVERTER INCLUDING THE REFERENCE VOLTAGE TERMS ...30

FIGURE 3.1-1 - ELECTRICAL BLOCK DIAGRAM-SCHEMATIC FOR TWO M-S CONTROL PARALLELED CONVERTERS.......34

FIGURE 3.1-2- CONTROL SYSTEMS BLOCK DIAGRAM-FOR TWO M-S CONTROL PARALLELED CONVERTER - RESISTIVE

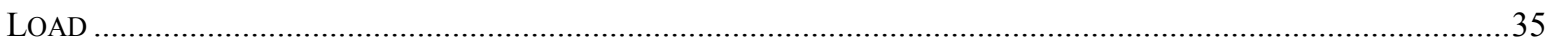

FIGURE 3.1-3- CONTROL SYSTEMS BLOCK DIAGRAM-FOR TWO M-S CONTROL PARALLELED CONVERTER -

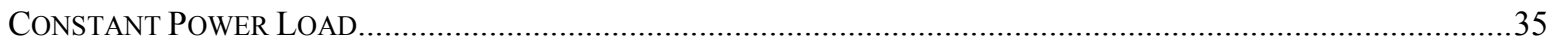

FIGURE 5.1-1 - CONTROL SCHEME USED FOR PARALLELING $N$ CONVERTER MODULES WITH MSC............................51

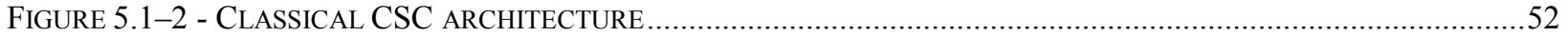

FIGURE 5.1-3 - FUZZY LOGIC IMPLEMENTATION OF CSC ................................................................................

FIGURE 5.1-4 - MEMBERSHIP FUNCTIONS AND RULE-BASED INFERENCE FOR THE FUZZY LOGIC CONTROLLER IN FIGURE

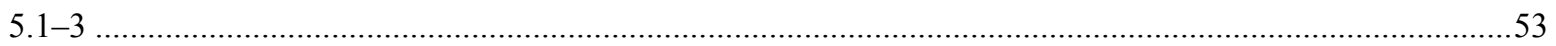

FIGURE 5.1-5 - SMALL SIGNAL LOOP GAINS FOR A CLASSICAL DESIGN AND IMPROVED PID DESIGN.........................56

FIGURE 5.1-6 - STEP LOAD RESPONSES FOR 50\% AND 90\% OF THE NOMINAL LOAD: (A)-SYSTEM WITH THE CLASSICAL

CSC IN FIGURE 5.1-2; (B)-SYSTEM EMPLOYING A PID CSC; (C)-SYSTEM USING A FUZZY LOGIC CSC ............57

Figure 5.1-7 - CSC CONTROL EFFORT (AVERAGED DUTY CYCLES): (A)-WiTH THE CLASSICAL CSC IN FIGURE 5.1-6;

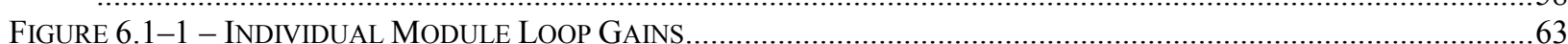

FIGURE 6.2-1 - SMALL SIGNAL LOOP GAINS FOR THE CLASSICAL DESIGN AND AN IMPROVED PID DESIGN .................65

FIGURE 6.2-2 - STEP LOAD RESPONSES FOR 25\% AND 50\% OF NOMINAL LOAD: (A)-SYSTEM WITH THE CLASSICAL

CSC IN FIGURE 5.1-2; (B)-SYSTEM EMPLOYING A PID CSC; (C)-SYSTEM USING A FUZZY LOGIC CSC ..........66

FIGURE 6.2-3 - STEP LOAD RESPONSES FOR 25\% AND 75\% OF NOMINAL LOAD: (A)-SYSTEM WITH THE CLASSICAL

CSC IN FIGURE 5.1-2; (B)-SYSTEM EMPLOYING A PID CSC; (C)-SYSTEM USING A FUZZY LOGIC CSC ..........67

FIGURE 6.2-4 - CSC CONTROL EFFORT FOR 25\% AND 75\% LOAD STEPS: (A)-SYSTEM WITH THE CLASSICAL CSC;

(B)-SYSTEM EMPLOYING A PID CSC; (C)-SYSTEM USING A FUZZY LOGIC CSC ...........................................68

FIGURE 6.2-5 - LYAPUNOV STABILITY ADAPTATION WAVEFORMS: (A)-STAND ALONE FUZZY LOGIC CONTROLLER;.69

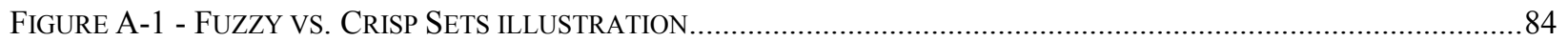

FIGURE A-2 - FUZZY MEMBERSHIP FUNCTIONS ILLUSTRATION....................................................................8

Figure A-3 - MEMBERShIP FunCtIONS AND TABLE FOR THE FuZZY IF-THEN Rule BASE IN (A-18) ...................90

FIGURE A-4 - CENTER OF GRAVITY DEFUZZIFIER ............................................................................

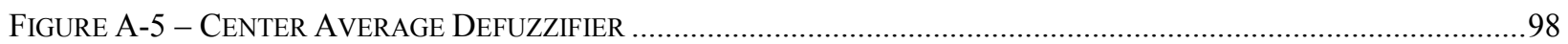

FIGURE A-6 - FUZZY LOGIC CONTROLLER USED FOR THE MS CSC IN THE THESIS ...........................................101 


\section{Chapter 1}

\section{INTRODUCTION}

\subsection{Background}

Paralleled DC/DC converter systems and, more generally, distributed power converter systems represent a major trend in today's power electronics research. Whereas the one time the stand-alone converter was the main feature, the single converter is now becoming an elementary cell for the next generation of power systems. The present focus on high-density integration and packaging will be naturally followed by a requirement for more advanced control methods, [1]. The evolution of power electronics is in many ways no different than the one of any other field, as it matures it generates new trends and potential spin-off directions.

In this context, paralleled DC/DC converter systems are becoming strong candidates for modern neuro-fuzzy control techniques due to their complex, nonlinear behavior, particularly for large load and line variations, [2]. Almost all existing control methods are based on simplified averaged linear models, [3] - [9] and, even in the case of a single unit, it has been shown that the large-signal response deviates significantly from the predictions of a small-signal model,

[10] - [12]. This is because, as known, the linearization around the steady-state operating point can only predict the plant behavior in the neighborhood of the respective operating point. Using linear analysis has however the advantages of being simple, explicit and well supported by all network analyzers used in the industry.

In all linear state space approaches, the input voltage and the load are treated more as small signal parameters in trying to assess the system's stability, rather than as large signal disturbances, which is a main performance issue with any real design, especially for a distributed, paralleled converters system. But, the circuit functions actually as a regulator and the loop dynamics, except for part of the noise spectrum, are exercised only in the case of a load and/or a line disturbance (step, impulse, etc.). Therefore, the control problem is one of a disturbance rejection and robustness behavior of a regulator module or system of paralleled regulator modules. 
Several attempts have been made to apply modern control theory for DC/DC converters, [13] - [20], including fuzzy logic control, [21] - [24]. The first ones recognized the importance of investigating the full, nonlinear averaged model. This comes out in a bilinear form and depending on the topology and control strategy, multiple equilibrium points can exist. One of the first control strategies based on the bilinear network models were in the form of Lyapunov control for a single module converter. They have the merit of introducing the advanced control concepts to the power electronics industry, but the latter had already been in a strong miniaturization trend and the traditional, PWM, OpAmp compensated converters were well established in the market.

Recognizing the variable topology network, for one switching cycle, couple of applications considered sliding mode control. Its implementation is almost always related to a hysteretic controller which had been explored before, with very good but similar, overall, results to the classical OpAmp controllers. However, sliding mode control offers an approach that is much closer to the real structure of the converter and can also be related to fuzzy logic control.

Once considered, fuzzy logic control started to be implemented by describing the converter's behavior with as many inference rules as possible and then creating a set of singleton gains for the control law. The gains were then adjusted considering both the voltage error and its derivative, together with the current's error, as necessary. This related the design of fuzzy controllers to using a PID controller as an expert for deriving the inference rules, which now could be done in a more precise manner. One of the ideas introduced in the beginning of the study used in this thesis was to first upgrade the classical, "type 2" or "type 3" compensators to a PID one and hence obtain the desired expert system for the fuzzy logic inference; in this way, an exact, small signal analysis and design can be done, prior to implementing the new fuzzy compensator.

A heuristic fuzzy logic approach becomes even more practical in the case of active control, e.g. master-slave control, for parallel operation of converters because of the difficulty in obtaining practical models for systems having a large number of feedback loops [25] - [33], [40], [41]. The complexity of current sharing for power converters fully justifies the advanced control methods of soft computing (neural, fuzzy and genetic) methods, whereas individual modules can, perhaps, be efficiently compensated with traditional techniques. In addition, since 
for distributed systems the volume constraints are less stringent than for a single module converter, both DSP and analog implementations are possible, [34], [35].

Lyapunov approaches become now practical as a way to stabilize any newly introduced control law. They have gained more and more grounds in conjunction with the modern control theory results and applications and can be considered either adaptively or in a supervisory fashion, [36] - [38]. 


\subsection{Objective and Outline}

Modern control of switching power converters is an interesting and challenging research topic. As mentioned above, the improved large signal performance and stability of power electronics systems has always been a main concern. Most times designers analyze in a linear, small signal formalism, each circuit individually in order to tune and than prescribe a scheme to accommodate a designated set of transients. The scope of this work is motivated by a need of new tools and techniques in order to explore and determine how one might do better.

The main objective of the research in this thesis is to introduce, conduct analysis and develop design methodologies for using emerging fuzzy logic control for improved large signal performance of paralleled DC/DC converter systems. In particular, this study develops easy to implement nonlinear fuzzy logic control algorithms that provide satisfactory global behavior while maintaining stability through a Lyapunov descent path, either in an adaptive or supervisory fashion.

Chapter 2 integrates and expands the present literature models in a modern, disturbance rejection and robustness framework. First, the bilinear structure of the converter's averaged model is proved using a rigorous Taylor series approach, newly applied for DC/DC modules. Then, the unterminated model, [17], [31], is introduced in a new, state space formalism, creating thus a disturbance vector with the load current, $i_{o}$ and input voltage, $v_{i n}$, as its components. This in turn offers the possibility of a new, straightforward, modern control systems modeling. Thus, mathematical simulators can be employed for describing and developing converter systems in a way that allows emerging control techniques to be used. Using Spice based circuit simulators alone, even though it offers a high power circuit check-up tool, is most times insufficient for developing a control strategy contribution in a highly interconnected, nonlinear system. The complete converter model has now a disturbance rejection formalism model, ready to be implemented in Matlab; since we are tackling the output current sharing problem, the input voltage disturbance is considered zero in the respective analysis. The input voltage comes explicitly, as an exogenous source and its rejection, well addressed by the present small signal methods, can be studied from a large signal angle in the new formalism as future research. 
The next contribution is to then include the load resistance into the plant matrix and observe its structure, carefully. Considering an ideal, loss-less converter, one can observe that the load resistance is only present in the last terms of the main diagonal of the " $\mathbf{A}$ " matrix and in the "B", column matrix. Hence, it can be factored out in a robustness, plant deviation factor, "L", which is introduced at the load step instant, for example. This shows the true nature of the system and of its response: it is the plant structure that changes, showing now its complex nature. This was slightly missed in the existing models in the literature and often, a step load is verified in practice, incorrectly, by a step in the reference voltage. Subsequent analyses are therefore done using the ideal model and the new control strategy verification is done employing the unterminated, complete converter model, including parasitics. This covers the usual small signal design, which should be conducted in detail, at the converter level, with the established small signal methods, prior of integrating the converter module in the paralleled system. As normal, at the system level, one starts the analysis and design considering that properly designed individual converters are given. In addition, the converter parasitics always add damping stability wise, the loss-less case study is a worst case design, especially from a Lyapunov approach standpoint.

Chapter 3 applies and extends the newly introduced formalism in Chapter 2, with detailing the Master-Slave (MS) paralleled system. This also lays the foundation for current sharing fuzzy logic control by expliciting now in detail the complex, interconnected nature of this type of systems.

MS paralleled systems, in a dedicated or rotating master scheme, cover - and can be equivalent - with a wide range of distributed power systems. The Current Sharing Error (CSE), i.e., the difference between the output currents of the master and slave converters, is fed back to the CSC whose output is then added to the reference input of the slave module. Normally, this input is on zero in terms of a dynamic input, for the individual DC/DC regulator.

Next, it is shown that the control problem in this case is a tracking problem of the slave following the master's current. The error fed back to the Current Sharing Compensator (CSC) is directly proportional with the error in the two plants output voltages, ideally equal with the second component of the converter's state vector. This is intuitive engineering wise since in practice the slave converter becomes a voltage controlled current source while the system's 
output voltage dynamics are given by the response of the internal loops of the master converter. By drawing the Master Slave Controlled (MSC) parallel system's block diagram both from an electrical and from a modern control system's viewpoint, more light is brought into how the control problem can be tackled. In this way, a general, state space model for the MSC distributed system is created for the first time in the framework of a tracking problem.

Based on the previously introduced results, Chapter 4 addresses the stability task by examining an adaptive, Lyapunov energy function trajectory approach, for the paralleled system. Even though several schemes were considered for individual converters, they did not offer practicality due to the contradictory trends of miniaturization for the converter unit on one hand and the computational complexity of the advanced controllers, on the other hand. Since already properly designed converters are employed in the next higher assembly of the distributed system, the Lyapunov analysis has to consider the closed loop DC/DC converter, compensated by a regular OpAmp controller, as done in the industry. This adds complexity to the Lyapunov function, chosen as the converter's state energy and especially to determining the closed form of its derivative.

The existence of an adaptive Lyapunov energy descent trajectory is proven considering the $\mathrm{DC} / \mathrm{DC}$ converter as general nonlinear plant, and applying an input-output linearization in order to highlight the CSE, assuming a FLC, [36] - [39]. The proof could be also used as a design method but is to general to comprise each practical case without a very long and cumbersome gain tweaking process. However, having proved the existence of such trajectory, the closed loop plant energy function can be written as mentioned above and then a control law can be inferred based on each specific topology.

Chapters 5 introduces fuzzy logic control as a new strategy for improved large signal performance for the current sharing loop of MSC paralleled DC/DC converters. The results obtained in the previous chapters are, from the converter's side, the framework used to embed the fuzzy logic reasoning and control law. Several attempts have been made to come up with emerging fuzzy logic control for one DC-DC converter, which deal naturally with nonlinearities due to their universal approximator feature. No study has been made though for applying the same concept in the more complicated parallel system, where heuristics would be even more 
justified in order to overcome the complexity in modeling the distributed system. In this regard, the work here also offers the novelty of a first study in that direction.

The approach is to first introduce an improved PID as an upgrade for the existing "Type 2" OpAmp compensators in the industry and then to employ the PID as an expert system in developing the rules for the Fuzzy Logic Controller (FLC). Based on the usual action of a PD cell, by looking at its output range as a function of the input range, a rule-based inference is chosen for each consequent using a simplified " $i$ th height" rule corresponding to consequent $\# i$. Further optimization in partitioning the input spaces as well as using neuro-fuzzy techniques in an adaptive grid of controllers can be considered as future research work.. A substantial improvement in the large signal, transient, performance and control effort will be obtained, as shown in Chapter 6, which depicts the design and comparative simulated results.

Chapter 6 gives simulated results for applying a FLC to a system of three Peak Current Mode Control (PCMC) boost converters with different parasitics and reference set-points, as given by the usual fabrication tolerances. The fuzzy logic implementation could be easily embedded in the existing schemes in the industry and offers an improved nonlinear, large signal, behavior. The almost identical responses for three step load cases, $25 \%, 50 \%$ and $75 \%$, when using the fuzzy control approach shows the capability of fuzzy control to deal naturally with the nonlinear character of a distributed system. The low performance index in the traditional cases is due to the fact that a conventional linear controller, designed only on a small signal model, is valid only near the operating point and not for the entire plant trajectory. Also, one can readily see that the fuzzy controller gives the minimal needed effort to control the system current sharing. The classical OpAmp compensator is the slowest and requires the most control effort; the PID controller gives an intermediary performance. In the case of a high gain, potentially oscillating system, a Lyapunov descent path supervisory controller, as mentioned above, was used with successful results. On and off-line gain adjustments are possible, based on each practical application.

Thesis Contributions, concluding remarks and suggestions for future research are given in Chapter 7. The two Appendices detail basic elements of fuzzy logic theory and Lyapunov stability. 


\section{Chapter 2}

\section{LARGE SIGNAL, ROBUSTNESS AND DISTURBANCE REJECTION ORIENTED MODELING FOR DC/DC CONVERTERS}

\subsection{Review of the Main Literature Results}

The basic DC-to-DC power conversion function of switching converters is achieved by repetitive switching between two linear networks consisting of storage elements, inductors and capacitors. As known, [3]-[9], state space averaging and linearization are analytical approximation techniques that allow switching regulators to be represented as linear systems. The averaging allows the switched, discontinuous, system to be approximated as a continuos, nonlinear, large signal model and then linearization around the DC steady state operating point is applied in order to obtain a small signal, linear model. Thus, in the small signal analysis, all the results of the linear control systems can be applied, provided that the perturbation in the system is small enough.. For large signal perturbations, [10]-[12], so important for time domain performance, the linear models prove less accurate, especially for paralleled systems. In all present state space approaches, both the input voltage and load, are treated mostly as parameters rather than as disturbances. But, the circuit functions actually as a regulator and, except for part of the noise spectrum, the loop dynamics are exercised only in the case of a load and/or a line disturbance (step, impulse, etc.). Hence, the control problem is one of disturbance rejection and robustness of a regulator or a system of paralleled regulators.

Therefore, working with the "unterminated" model, [17], [31], in which the output current is another variable, is a good way to approach the problem. This is also motivated by the way in which the load comes into the system's model. All previous state space approaches consider a resistive load, $i_{o}=v_{d} / R_{l d}$ and write $v_{o}$ in terms of the states $\left[\begin{array}{ll}i_{l} & v_{c}\end{array}\right]^{\prime}-$ inductor current and capacitor voltage. Hence, they get a model that has $R_{l d}$ in the associated matrices and no $i_{o}$ 
terms. But, in many cases, especially for distributed systems, the loads are more complex and an unterminated model formalism becomes necessary. So far, this has only been detailed in a transfer functions, $s$ domain, type of analysis. In the next paragraphs, such a formalism is introduced using state space modeling and considering the large signal problem in a robustness and disturbance rejection framework.

First, the classical, resistive load model will be reviewed. Without loss of generality, considering PWM CCM, any switching DC-DC converter, terminated to the resistive load $R_{l d}$, can be described by the state space equations of the two linear networks corresponding to the two switching stages. For example, considering the boost converter in Figure 2.1-1, we have

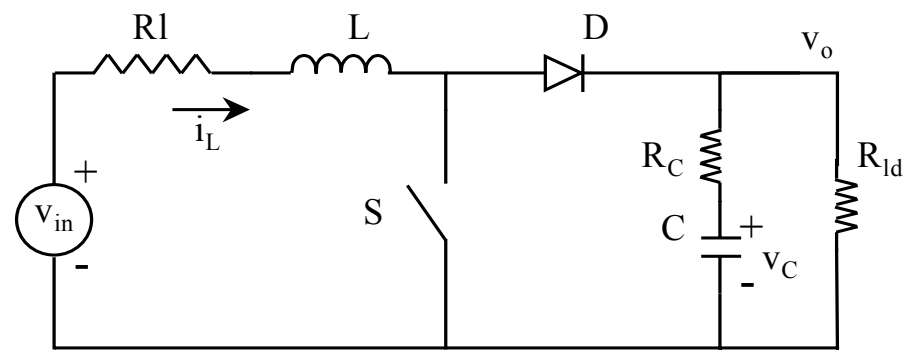

(a)

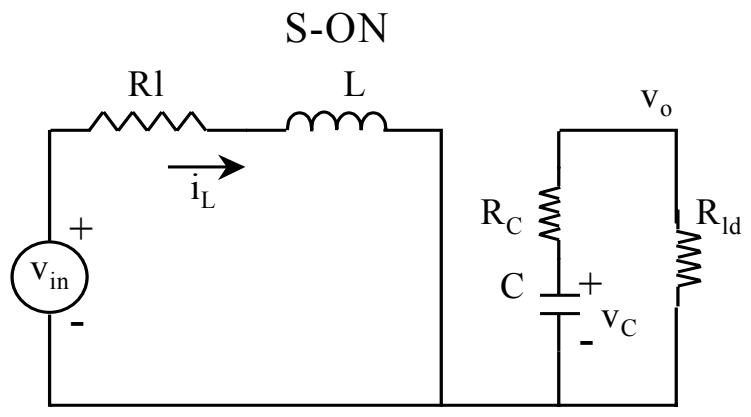

(b)

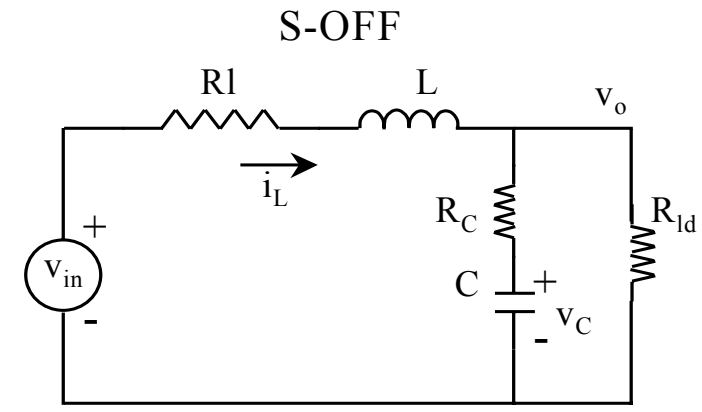

(c)

Figure 2.1-1 - Boost Converter in CCM using the resistive load terminated model

When the switch is ON, during the interval $d \cdot T_{s}$, Figure $2.1-1-(\mathrm{b})$ :

$$
\begin{aligned}
& \dot{\mathbf{x}}=\left[\begin{array}{c}
\dot{i}_{L} \\
\dot{v}_{c}
\end{array}\right]=\left[\begin{array}{cc}
-\frac{R_{L}}{L} & 0 \\
0 & -\frac{1}{C} \cdot \frac{1}{R_{l d}+R_{C}}
\end{array}\right] \cdot\left[\begin{array}{c}
i_{L} \\
v_{c}
\end{array}\right]+\left[\begin{array}{c}
\frac{1}{L} \\
0
\end{array}\right] \cdot v_{i n}=\mathbf{A}_{\mathbf{1}} \cdot \mathbf{x}+\mathbf{E}_{\mathbf{1 x}}^{\mathbf{v}_{\text {in }}} \cdot v_{i n} \\
& v_{o} \equiv y=\left[0 \frac{R_{l d}}{R_{l d}+R_{C}}\right] \cdot\left[\begin{array}{l}
i_{L} \\
v_{c}
\end{array}\right]=\mathbf{C}_{\mathbf{1}} \cdot \mathbf{x}
\end{aligned}
$$

and when the switch is OFF, during the interval $(1-d) \cdot T_{s}=d^{\prime} \cdot T_{s}$, Figure 2.1-1 - (c): 


$$
\begin{aligned}
& \dot{\mathbf{x}}=\left[\begin{array}{l}
\dot{i}_{L} \\
\dot{v}_{c}
\end{array}\right]=\left[\begin{array}{cc}
-\frac{1}{L} \cdot\left(R_{L}+\frac{R_{l d} \cdot R_{C}}{R_{l d}+R_{C}}\right) & -\frac{1}{L} \cdot \frac{R_{l d}}{R_{l d}+R_{C}} \\
\frac{1}{C} \cdot \frac{R_{l d}}{R_{l d}+R_{C}} & -\frac{1}{C} \cdot \frac{1}{R_{l d}+R_{C}}
\end{array}\right] \cdot\left[\begin{array}{c}
i_{L} \\
v_{c}
\end{array}\right]+\left[\begin{array}{c}
\frac{1}{L} \\
0
\end{array}\right] \cdot v_{i n}=\mathbf{A}_{2} \cdot \mathbf{x}+\mathbf{E}_{2 \mathbf{x}}^{\mathbf{v}_{\text {in }}} \cdot v_{i n} \\
& v_{o} \equiv y=\left[\begin{array}{ll}
\frac{R_{l d} \cdot R_{C}}{R_{l d}+R_{C}} & \frac{R_{l d}}{R_{l d}+R_{C}}
\end{array}\right] \cdot\left[\begin{array}{l}
i_{L} \\
v_{c}
\end{array}\right]=\mathbf{C}_{\mathbf{2}} \cdot \mathbf{x}
\end{aligned}
$$

The classical small signal analysis, [3], uses the "straight line" approximation of the fundamental matrix, $\mathbf{e}^{\mathbf{A} \cdot \mathbf{t}} \cong \mathbf{I}+\mathbf{A} \cdot t$, to obtain a unique, linear, averaged model, for the entire switching cycle. The same averaging process is employed to derive the discrete averaged model, [8], where it is still needed to have a single model for one whole switching cycle. The matrices of this model are obtained to be simply the average of each switching half-cycle model matrix, weighed with the duty cycle, $D$ at the DC operating point, $\mathbf{X}_{\mathbf{0}}, Y, V_{i n}$. This linear approximation works well as long as the perturbation is small, around the steady state operation point and the ratio between the equivalent corner frequency of the output filter and the switching frequency is much smaller than unity, $f_{c} / f_{s}<<1$.

Thus, one obtains first the continuous, nonlinear (bilinear) model

$$
\begin{aligned}
& \dot{\mathbf{x}}=\mathbf{A} \cdot \mathbf{x}+\mathbf{B} \cdot u+\mathbf{E}_{\mathbf{x}}^{\mathbf{v}_{\text {in }}} \cdot v_{i n}+\mathbf{\Delta} \mathbf{A} \cdot \mathbf{x} \cdot u+\Delta \mathbf{E}_{\mathbf{x}}^{\mathbf{v}_{\text {in }}} \cdot v_{i n} \cdot u \\
& v_{o} \equiv y=\mathbf{C} \cdot \mathbf{x}+\mathbf{D} \cdot u+\Delta \mathbf{C} \cdot \mathbf{x} \cdot u
\end{aligned}
$$

with

$$
\begin{aligned}
& \mathbf{A}=\mathbf{A}_{\mathbf{1}} \cdot D+\mathbf{A}_{\mathbf{2}} \cdot(1-D), \quad \mathbf{B}=\Delta \mathbf{A} \cdot \mathbf{X}_{\mathbf{0}}+\Delta \mathbf{E}_{\mathbf{x}}^{\mathbf{v}_{\text {in }}} \cdot V_{i n}, \quad \mathbf{C}=\mathbf{C}_{\mathbf{1}} \cdot D+\mathbf{C}_{\mathbf{2}} \cdot(1-D) \\
& \mathbf{D}=\Delta \mathbf{C} \cdot \mathbf{X}_{\mathbf{0}}, \quad \mathbf{E}_{\mathbf{x}}^{\mathbf{v}_{\text {in }}}=\mathbf{E}_{1 \mathbf{x}}^{\mathbf{v}_{\text {in }}} \cdot D+\mathbf{E}_{2 \mathbf{x}}^{\mathbf{v}_{\text {in }}} \cdot(1-D), \mathbf{X}_{\mathbf{0} r}=-\mathbf{A}^{-1} \cdot \mathbf{E}_{\mathbf{x}}^{\mathbf{v}_{\text {in }}} \cdot V_{i n}
\end{aligned}
$$

and $\Delta$ denoting the difference between the two respective quantities. For the boost converter above, the detailed model resulting from (2.1-3) would be

$$
\mathbf{X}_{\mathbf{0} r}=\left[\begin{array}{c}
\frac{R_{l d}}{R^{2}} \cdot V_{i n} \\
\frac{1}{1-D} \cdot V_{i n} \cdot \frac{(1-D)^{2} \cdot R_{l d}}{R^{\prime}}
\end{array}\right]
$$




$$
\begin{aligned}
& \dot{\mathbf{x}}=\left[\begin{array}{cc}
-\frac{R_{L}+\left(R_{l d} \| R_{C}\right) \cdot(1-D)}{L} & -\frac{R_{l d} \cdot(1-D)}{\left(R_{l d}+R_{C}\right) \cdot L} \\
\frac{R_{l d} \cdot(1-D)}{\left(R_{l d}+R_{C}\right) \cdot C} & -\frac{1}{\left(R_{l d}+R_{C}\right) \cdot C}
\end{array}\right] \cdot \mathbf{x}+\left[\begin{array}{c}
\frac{1}{L} \cdot \frac{R_{l d}}{R^{\prime}} \cdot \frac{(1-D) \cdot R_{l d}+R_{C}}{R_{l d}+R_{C}} \\
-\frac{R_{l d}}{R^{\prime}} \cdot \frac{1}{\left(R_{l d}+R_{C}\right) \cdot C}
\end{array}\right] \cdot V_{i n} \cdot u+\left[\begin{array}{c}
\frac{1}{L} \\
0
\end{array}\right] \cdot v_{i n}+\left[\begin{array}{cc}
\frac{R_{l d} \| R_{C}}{L} & \frac{R_{l d}}{\left(R_{l d}+R_{C}\right) \cdot L} \\
-\frac{R_{l d}}{\left(R_{l d}+R_{C}\right) \cdot C} & 0
\end{array}\right] \cdot \mathbf{x} \cdot u \\
& y=\left[\begin{array}{ll}
\left(R_{l d} \| R_{C}\right) \cdot(1-D) & \frac{R_{l d}}{\left(R_{l d}+R_{C}\right) \cdot C}
\end{array}\right] \cdot \mathbf{x}-V_{i n} \cdot \frac{R_{l d} \| R_{C}}{R^{\prime}} \cdot u+\left[\begin{array}{ll}
-\left(R_{l d} \| R_{C}\right) & 0
\end{array}\right] \cdot \mathbf{x} \cdot u
\end{aligned}
$$

with

$$
R^{\prime}=R_{L}+\left(R_{l d} \| R_{C}\right) \cdot D \cdot(1-D)+(1-D)^{2} \cdot R_{l d}
$$

Then, by keeping the linear terms, the small signal model can be obtained and one can calculate the salient Laplace transfer functions and use Bode plots techniques for stability analysis. This is very useful in industry, because the vast majority of commercially available network analyzers offer excellent gain, phase and impedance measurements.

When the nonlinear, large signal model was first analyzed in detail, [10], it was shown how the converter can substantially deviate from the predictions of the small signal model. The averaged converter model comes out as bilinear and, depending on the design, may have multiple equilibrium points, both real and virtual. Therefore it is very important to consider the nonlinear, large signal performance as a strong design goal. The next paragraph offers a proof using another approach that employs series expansion and links the model to the ideas proposed in this study. 


\subsection{The DC/DC Converter as a Regulator under Line and Load Disturbances}

Consider now the same boost converter above, but using the unterminated model, where the output current is the load disturbance variable, Figure 2.2-1.

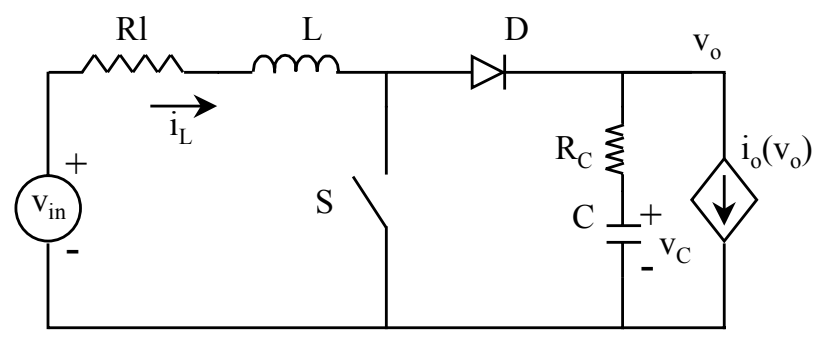

(a)

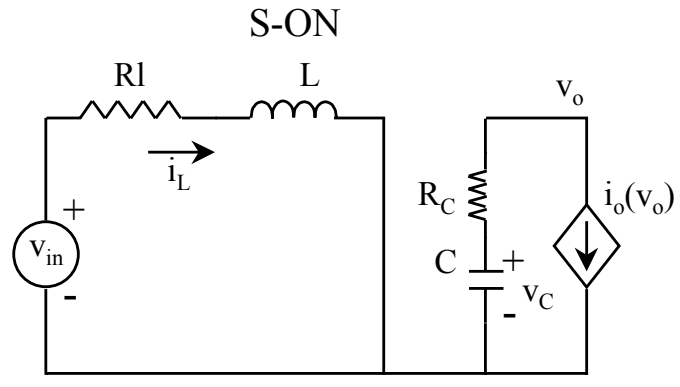

(b)

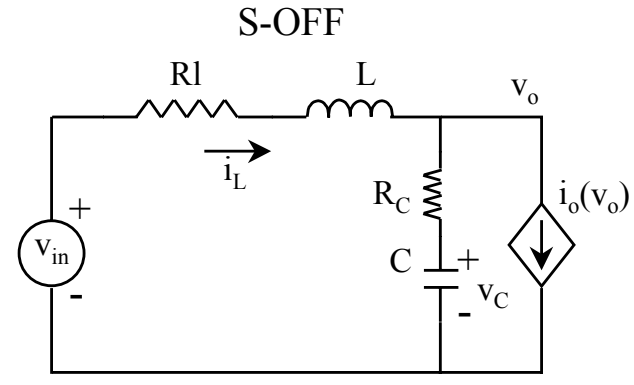

(c)

Figure 2.2-1 - Boost Converter in CCM using the Unterminated Model

Continuing the case in the previous paragraph, the model equations are

$$
\begin{aligned}
& {\left[\begin{array}{l}
\dot{i}_{L} \\
\dot{v}_{C}
\end{array}\right]=\left[\begin{array}{cc}
-\frac{R_{l}}{L} & 0 \\
0 & 0
\end{array}\right] \cdot\left[\begin{array}{c}
i_{L} \\
v_{C}
\end{array}\right]+\left[\begin{array}{c}
\frac{1}{L} \\
0
\end{array}\right] \cdot v_{i n}+\left[\begin{array}{c}
0 \\
-\frac{1}{C}
\end{array}\right] \cdot i_{o}=\mathbf{A}_{\mathbf{1}} \cdot \mathbf{x}+\mathbf{E}_{\mathbf{1 x}}^{\mathbf{v}_{\mathbf{i n}}} \cdot v_{i n}+\mathbf{E}_{\mathbf{1 x}}^{\mathbf{i}_{\mathbf{x}}} \cdot i_{o}} \\
& v_{o}=\left[\begin{array}{ll}
0 & 1
\end{array}\right] \cdot\left[\begin{array}{c}
i_{L} \\
v_{C}
\end{array}\right]-R_{C} \cdot i_{o}=\mathbf{C}_{\mathbf{1}} \cdot \mathbf{x}+\mathbf{E}_{\mathbf{1 y}}^{\mathbf{i}_{\mathbf{y}}} \cdot i_{o}
\end{aligned}
$$

for the ON time and 


$$
\begin{aligned}
& {\left[\begin{array}{l}
i_{L} \\
\dot{v}_{C}
\end{array}\right]=\left[\begin{array}{cc}
-\frac{R_{l}+R_{C}}{L} & -\frac{1}{L} \\
\frac{1}{C} & 0
\end{array}\right] \cdot\left[\begin{array}{c}
i_{L} \\
v_{C}
\end{array}\right]+\left[\begin{array}{c}
\frac{1}{L} \\
0
\end{array}\right] \cdot v_{i n}+\left[\begin{array}{c}
\frac{R_{C}}{L} \\
-\frac{1}{C}
\end{array}\right] \cdot i_{o}=\mathbf{A}_{2} \cdot \mathbf{x}+\mathbf{E}_{2 \mathbf{x}}^{\mathbf{v}_{\text {in }}} \cdot v_{i n}+\mathbf{E}_{2 \mathbf{x}}^{\mathbf{i}_{\mathbf{x}}} \cdot i_{o}} \\
& v_{o}=\left[\begin{array}{ll}
R_{C} & 1
\end{array}\right] \cdot\left[\begin{array}{c}
i_{L} \\
v_{C}
\end{array}\right]-R_{C} \cdot i_{o}=\mathbf{C}_{2} \cdot \mathbf{x}+\mathbf{E}_{2 \mathbf{y}}^{\mathbf{i}_{0}} \cdot i_{o}
\end{aligned}
$$

for the OFF time.

The same equations can be written in a compact form,

$$
\begin{aligned}
& \dot{\mathbf{x}}=\mathbf{A}_{\mathbf{1}} \cdot \mathbf{x}+\left[\begin{array}{ll}
\mathbf{E}_{\mathbf{1 x}}^{\mathbf{v}_{\text {in }}} & \mathbf{E}_{\mathbf{1 x}}^{\mathbf{i}_{\mathbf{o}}}
\end{array}\right] \cdot\left[\begin{array}{l}
v_{i n} \\
i_{o}
\end{array}\right]=\mathbf{A}_{\mathbf{1}} \cdot \mathbf{x}+\mathbf{E}_{\mathbf{1 x}} \cdot \mathbf{w} \\
& v_{o} \equiv y=\mathbf{C}_{\mathbf{1}} \cdot \mathbf{x}+\left[\begin{array}{ll}
0 & \mathbf{E}_{\mathbf{1 y}}^{\mathbf{i}_{\mathbf{o}}}
\end{array}\right] \cdot\left[\begin{array}{l}
v_{i n} \\
i_{o}
\end{array}\right]=\mathbf{C}_{\mathbf{1}} \cdot \mathbf{x}+\mathbf{E}_{\mathbf{1 y}} \cdot \mathbf{W}
\end{aligned}
$$

and respectively

$$
\begin{aligned}
& \dot{\mathbf{x}}=\mathbf{A}_{2} \cdot \mathbf{x}+\left[\begin{array}{ll}
\mathbf{E}_{2 \mathbf{x}}^{\mathbf{v}_{\mathbf{i n}}} & \mathbf{E}_{2 \mathbf{x}}^{\mathbf{i}_{0}}
\end{array}\right] \cdot\left[\begin{array}{l}
v_{i n} \\
i_{o}
\end{array}\right]=\mathbf{A}_{2} \cdot \mathbf{x}+\mathbf{E}_{2 \mathbf{x}} \cdot \mathbf{w} \\
& v_{o} \equiv y_{o}=\mathbf{C}_{2} \cdot \mathbf{x}+\left[\begin{array}{ll}
\mathbf{0} & \mathbf{E}_{2 \mathbf{y}}^{\mathbf{i}_{0}}
\end{array}\right] \cdot\left[\begin{array}{l}
v_{i n} \\
i_{o}
\end{array}\right]=\mathbf{C}_{2} \cdot \mathbf{x}+\mathbf{E}_{2 \mathbf{y}} \cdot \mathbf{W}
\end{aligned}
$$

Here, the disturbance vector, w, contains both the input voltage and the output (load) current, which is assumed constant over one switching cycle, a very good practical approximation. Since the output filter requirement for the straight line approximation mentioned above, $f_{c} / f_{s}<<1$, holds for most present designs, we obtain the solutions

$$
\begin{aligned}
& \mathbf{x}(t)=\left(\mathbf{I}+\mathbf{A}_{\mathbf{1}} \cdot t\right) \cdot \mathbf{x}(0)+\mathbf{E}_{\mathbf{1 x}} \cdot \mathbf{w} \cdot t, \\
& y(t)=\left(\mathbf{I}+\mathbf{C}_{\mathbf{1}} \cdot t\right) \cdot \mathbf{x}(0)+\mathbf{E}_{\mathbf{1 y}} \cdot \mathbf{w} \cdot t
\end{aligned}
$$

for $0 \leq t<d \cdot T_{s}$ and

$$
\begin{aligned}
& \mathbf{x}(t)=\left[\mathbf{I}+\mathbf{A}_{2} \cdot\left(t-d \cdot T_{s}\right)\right] \cdot \mathbf{x}\left(d \cdot T_{s}\right)+\mathbf{E}_{\mathbf{2 x}} \cdot \mathbf{w} \cdot\left(t-d \cdot T_{s}\right), \\
& y(t)=\left[\mathbf{I}+\mathbf{C}_{\mathbf{2}} \cdot\left(t-d \cdot T_{s}\right)\right] \cdot \mathbf{x}\left(d \cdot T_{s}\right)+\mathbf{E}_{2 \mathbf{y}} \cdot \mathbf{w} \cdot\left(t-d \cdot T_{s}\right)
\end{aligned}
$$

for $d \cdot T_{s} \leq t<T_{s}$. The combination of equations (2.2-5) - (2.2-8) yields the following expression for $x\left(T_{s}\right)$ and $y\left(T_{s}\right)$, respectively:

$$
\mathbf{x}\left(T_{s}\right)=\left[\mathbf{I}+T_{s} \cdot\left(d \cdot \mathbf{A}_{\mathbf{1}}+d^{\prime} \cdot \mathbf{A}_{\mathbf{2}}\right)\right] \cdot \mathbf{x}(0)+T_{s} \cdot\left(d \cdot \mathbf{E}_{\mathbf{1 x}}+d^{\prime} \cdot \mathbf{E}_{\mathbf{2 x}}\right) \cdot \mathbf{w}
$$




$$
y\left(T_{s}\right)=\left[\mathbf{I}+T_{s} \cdot\left(d \cdot \mathbf{C}_{\mathbf{1}}+d^{\prime} \cdot \mathbf{C}_{\mathbf{2}}\right)\right] \cdot \mathbf{x}\left(T_{s}\right)+T_{s} \cdot\left(d \cdot \mathbf{E}_{\mathbf{1 y}}+d^{\prime} \cdot \mathbf{E}_{\mathbf{2 y}}\right) \cdot \mathbf{w}
$$

After $n+1$ switching intervals, we have

$$
\begin{aligned}
& \mathbf{x}\left((n+1) \cdot T_{s}\right)=\mathbf{x}_{\mathbf{n}+\mathbf{1}}=\left[\mathbf{I}+T_{s} \cdot\left(d_{n} \cdot \mathbf{A}_{\mathbf{1}}+d_{n}^{\prime} \cdot \mathbf{A}_{\mathbf{2}}\right)\right] \cdot \mathbf{x}_{n}+T_{s} \cdot\left(d_{n} \cdot \mathbf{E}_{\mathbf{1} \mathbf{x}}+d_{n}^{\prime} \cdot \mathbf{E}_{\mathbf{2 x}}\right) \cdot \mathbf{w}_{\mathbf{n}} \\
& y\left((n+1) \cdot T_{s}\right)=y_{\mathbf{n}+\mathbf{1}}=\left[\mathbf{I}+T_{s} \cdot\left(d \cdot \mathbf{C}_{\mathbf{1}}+d_{n}^{\prime} \cdot \mathbf{C}_{\mathbf{2}}\right)\right] \cdot \mathbf{x}_{\mathbf{n}}+T_{s} \cdot\left(d_{n} \cdot \mathbf{E}_{\mathbf{1 y}}+d_{n}^{\prime} \cdot \mathbf{E}_{\mathbf{2 y}}\right) \cdot \mathbf{w}_{\mathbf{n}}
\end{aligned}
$$

which constitutes the discrete, nonlinear, averaged model for the system.

Since the DC/DC regulator is trying to regulate the output voltage at some constant value, e.g., $+5 \mathrm{~V}$, we need to shift the axes around the nominal, DC operating point, which occurs at

$$
\mathbf{x}_{\mathbf{n}+1}=\mathbf{x}_{\mathbf{n}}=\mathbf{X}_{0}, \quad y_{\mathbf{n}+1}=y_{\mathbf{n}}=Y_{0}, \quad d_{\mathbf{n}+1}=d_{\mathbf{n}}=D_{0}=D, \quad \mathbf{w}_{\mathbf{n}+1}=\mathbf{w}_{\mathbf{n}}=\mathbf{W}_{\mathbf{0}},
$$

so the quiescent operating point of the regulator is at the origin. In this way, the development of the discrete, averaged model would be complete. Although the discrete equations are well-suited for digital simulation, they are not fully relevant for in dept, analytical calculations. Moreover, due to the low pass filter at the output with a cut-off frequency much lower than the switching frequency, this is an energy processing system and not an informational system. It is then preferred to work with the continuous time model which can easily model the OpAmp compensator, etc. and offers physical insight into the problem. If one uses the Euler forwarddifferentiation approximation, the continuous derivative of the state can be estimated as

$$
\frac{d \mathbf{x}(t)}{d t}=\frac{\mathbf{x}_{\mathbf{n}+\mathbf{1}}-\mathbf{x}_{\mathbf{n}}}{T_{s}} .
$$

We obtain thus a continuous, nonlinear system

$$
\begin{aligned}
& \dot{\mathbf{x}}=\left[\mathbf{A}_{1} \cdot u+\mathbf{A}_{2} \cdot(1-u)\right] \cdot \mathbf{x}+\left[\mathbf{E}_{1 \mathbf{x}} \cdot u+\mathbf{E}_{2 \mathbf{x}} \cdot(1-u)\right] \cdot \mathbf{w} \\
& y=\left[\mathbf{C}_{\mathbf{1}} \cdot u+\mathbf{C}_{\mathbf{2}} \cdot(1-u)\right] \cdot \mathbf{x}+\mathbf{E}_{\mathbf{y}} \cdot \mathbf{w}
\end{aligned}
$$

where the duty cycle, $d$, was replaced using the modern control formalism notation with $u$ and we used the fact that $\mathbf{E}_{\mathbf{1 y}}=\mathbf{E}_{\mathbf{2 y}}=\left[0-\mathrm{R}_{\mathrm{C}}\right]$. It can now easily be seen that perturbing the model around the DC operating point is nothing else but considering the general formula of a Taylor expansion in the three variables $x, u, w$ : 


$$
\begin{aligned}
f(x, u, w)= & {\left[f\left(X_{0}, U_{0}, W_{0}\right)\right]_{=O P}+\left.\frac{\partial f}{\partial x}\right|_{O P} \cdot \underbrace{\left(x-X_{0}\right)}_{\text {new } x}+\left.\frac{\partial f}{\partial w}\right|_{O P} \cdot \underbrace{\left(w-W_{0}\right)}_{\text {new } w}+\left.\frac{\partial f}{\partial d}\right|_{O P} \cdot \underbrace{\left(u-U_{0}\right)}_{\text {new } u}+} \\
& +\left.\frac{\partial^{2} f}{\partial x \partial u}\right|_{O P} \cdot\left(x-X_{0}\right) \cdot\left(u-U_{0}\right)+\left.\frac{\partial^{2} f}{\partial x \partial w}\right|_{O P} \cdot\left(x-X_{0}\right) \cdot\left(w-W_{0}\right)+ \\
& +\left.\frac{\partial^{2} f}{\partial x \partial u}\right|_{O P} \cdot\left(x-X_{0}\right) \cdot\left(u-U_{0}\right)+\left.\frac{1}{2 !} \cdot \frac{\partial^{2} f}{\partial x^{2}}\right|_{O P} \cdot\left(x-X_{0}\right)^{2}+ \\
& +\left.\frac{1}{2 !} \cdot \frac{\partial^{2} f}{\partial w^{2}}\right|_{O P} \cdot\left(w-W_{0}\right)^{2}+\left.\frac{1}{2 !} \cdot \frac{\partial^{2} f}{\partial u^{2}}\right|_{O P} \cdot\left(u-U_{0}\right)^{2}+ \\
& +\left.\frac{\partial^{3} f}{\partial x \partial u \partial w}\right|_{O P} \cdot\left(x-X_{0}\right) \cdot\left(u-U_{0}\right) \cdot\left(w-W_{0}\right)+\ldots
\end{aligned}
$$

The differences in parentheses are the dynamics of the perturbation from the nominal Operating Point (OP), $\left[V_{I N}, I_{O}\right]=\mathbf{W}_{\mathbf{0}}, D=U_{0}$, and $\mathbf{X}_{\mathbf{0}}$ and will be our default new variables, i.e. $x, u, w$; the same applies to the output, $y$, around its DC value $V_{0}=Y_{0}$. As it can be seen from (2.2-15), only the terms in the DC OP, the first derivatives and the second derivatives not in the same variable are non-zero. Hence, we obtain a model in the bilinear form,

$$
\begin{aligned}
& \dot{\mathbf{x}}+\left(\dot{\mathbf{X}}_{\mathbf{0}}\right)_{" O P(=0)^{\prime \prime}}=\left\{\mathbf{A} \cdot \mathbf{X}_{\mathbf{0}}+\mathbf{E}_{\mathbf{x}} \cdot \mathbf{W}_{\mathbf{0}}\right\}_{" O P(=0) "}+\left\{\mathbf{A} \cdot \mathbf{x}+(\mathbf{B} \cdot u)_{" B \cdot u^{\prime \prime}}+\left(\mathbf{E}_{\mathbf{x}} \cdot \mathbf{W}\right)_{" \text { Dist" }}\right\}_{\text {Lin }}+
\end{aligned}
$$

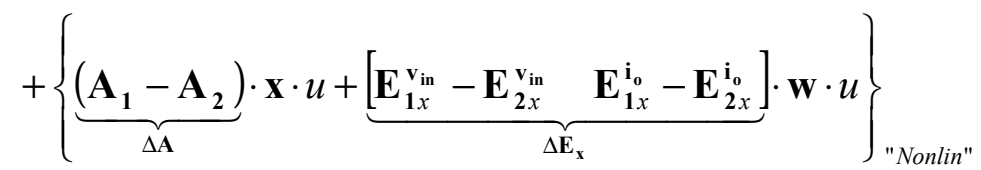

$$
\begin{aligned}
& y+\left(Y_{O}\right)_{" O P\left(=V_{o}\right) "}=\left\{\mathbf{C} \cdot \mathbf{X}_{\mathbf{0}}+\mathbf{E}_{\mathbf{y}} \cdot \mathbf{W}_{\mathbf{0}}\right\}_{" O P\left(=V_{o}\right) "}+\left\{\mathbf{C} \cdot \mathbf{x}+(\mathbf{D} \cdot u)_{" D \cdot u^{\prime \prime}}+(\mathbf{E} \mathbf{y} \cdot \mathbf{w})_{" D i s t "}\right\}_{" L i n "}+ \\
& +\{\underbrace{\left(\mathbf{C}_{\mathbf{1}}-\mathbf{C}_{2}\right)}_{\Delta \mathbf{C}} \cdot \mathbf{x} \cdot u+(\underbrace{\left[\begin{array}{ll}
\mathbf{0} & \mathbf{E}_{\mathbf{1 y}}^{\mathbf{i}_{\mathbf{o}}}-\mathbf{E}_{\mathbf{2 y}}^{\mathbf{i}_{\mathbf{o}}}
\end{array}\right]}_{\Delta \mathbf{E}_{\mathbf{y}}} \cdot \mathbf{w} \cdot u)_{=0}\}_{\text {"Nonlin" }}
\end{aligned}
$$

with

$$
\begin{aligned}
& \mathbf{A}=\mathbf{A}_{\mathbf{1}} \cdot D+\mathbf{A}_{\mathbf{2}} \cdot(1-D), \mathbf{B}=\Delta \mathbf{A} \cdot \mathbf{X}_{\mathbf{0}}+\Delta \mathbf{E}_{\mathbf{x}} \cdot \mathbf{W}_{\mathbf{0}}, \mathbf{C}=\mathbf{C}_{\mathbf{1}} \cdot D+\mathbf{C}_{\mathbf{2}} \cdot(1-D) \\
& \mathbf{D}=\Delta \mathbf{C} \cdot \mathbf{X}_{\mathbf{0}}+\left(\mathbf{E}_{\mathbf{1} y}^{\mathbf{i}_{\mathbf{o}}}-\mathbf{E}_{\mathbf{2} y}^{\mathbf{i}_{\mathbf{o}}}\right) \cdot I_{O}=\Delta \mathbf{C} \cdot \mathbf{X}_{\mathbf{0}}, \operatorname{since} \mathbf{E}_{\mathbf{1} y}^{\mathbf{i}_{\mathbf{o}}}=\mathbf{E}_{2 y}^{\mathbf{i}_{\mathbf{o}}}=-R_{C}(\mathrm{ESR}) \\
& \mathbf{E}_{\mathbf{x}}=\mathbf{E}_{\mathbf{1 x}} \cdot D+\mathbf{E}_{\mathbf{1} \mathbf{x}} \cdot(1-D), \quad \mathbf{E}_{\mathbf{y}}=\mathbf{E}_{\mathbf{1 y}} \cdot D+\mathbf{E}_{\mathbf{1 y}} \cdot(1-D)=-R_{C},
\end{aligned} .
$$

where $\mathbf{X}_{\mathbf{0}}=-\mathbf{A}^{-1} \cdot \mathbf{E}_{\mathbf{x}} \cdot \mathbf{W}_{\mathbf{0}}$ 
On short,

$$
\begin{aligned}
& \dot{\mathbf{x}}=\mathbf{A} \cdot \mathbf{x}+(\Delta \mathbf{A} \cdot \mathbf{x}+\mathbf{B}) \cdot u+\left(\Delta \mathbf{E}_{\mathbf{x}} \cdot u+\mathbf{E}_{\mathbf{x}}\right) \cdot \mathbf{w}=f_{1}(\mathbf{x})+g_{1}(\mathbf{x}) \cdot u+p_{1}(u) \cdot \mathbf{w}, \\
& y=\mathbf{C} \cdot \mathbf{x}+(\Delta \mathbf{C} \cdot \mathbf{x}+\mathbf{D}) \cdot u+\mathbf{E}_{\mathbf{y}} \cdot \mathbf{w}=f_{2}(\mathbf{x})+g_{2}(\mathbf{x}) \cdot u+\mathbf{E}_{\mathbf{y}} \cdot \mathbf{w}
\end{aligned}
$$

This result is the same as the one obtained by employing the elementary method of writing the equations using the perturbations around the DC operating point shifted axes, $\mathbf{x}+\mathbf{X}_{\mathbf{0}}$, $\mathbf{w}+\mathbf{W}_{\mathbf{0}}$, etc., and maintaining the second order terms; the linear model would only keep the first order terms. However, that approach does not offer the necessary insight to properly formalize the control issues noted above.

For the rest of the developments in this study, the boost converter in Figure 2.1-1 and Figure 2.2-1 will be used as an example since it offers both the nonlinear terms and a nonminimal phase (RHZ) behavior and therefore it is a good case to verify the validity and performance of the new control strategy. Comparative references will be given also for the buck and buck-boost converters; the Cuk, Sepic and Dual Sepic (Zeta) fourth order converters are combinations of the first three and can be treated in a similar manner; the same stands for the DCM case, for each converter.

If we reconsider the boost converter in Figure 2.2-1, average the system over one switching cycle and perturb it around the DC operating point, we obtain for the DC steady state solution and systems dynamics, respectively,

$$
\mathbf{X}_{\mathbf{0}}=\left[\begin{array}{c}
\frac{1}{1-D} \cdot I_{o} \\
\frac{1}{1-D} \cdot V_{\text {in }}-\left[\frac{D}{1-D} \cdot R_{C}+\frac{R_{L}}{(1-D)^{2}}\right]
\end{array}\right]
$$

and

$$
\begin{aligned}
& \dot{\mathbf{x}} \equiv\left[\begin{array}{c}
\dot{i}_{L} \\
\dot{v}_{C}
\end{array}\right]=\left[\begin{array}{cc}
-\frac{R_{l}+R_{C} \cdot(1-D)}{L} & -\frac{1-D}{L} \\
\frac{1-D}{C} & 0
\end{array}\right] \cdot \mathbf{x}+\left[\begin{array}{c}
\frac{V_{i n} \cdot(1-D)-I_{o} \cdot R_{l}}{L \cdot(1-D)^{2}} \\
-\frac{I_{o}}{C \cdot(1-D)}
\end{array}\right] \cdot u+\left[\begin{array}{cc}
\frac{1}{L} & \frac{R_{C} \cdot(1-D)}{L} \\
0 & -\frac{1}{C}
\end{array}\right] \cdot \mathbf{W}+\left[\begin{array}{cc}
\frac{R_{C}}{L} & \frac{1}{L} \\
-\frac{1}{C} & 0
\end{array}\right] \cdot \mathbf{x} \cdot u+ \\
& +\left[\begin{array}{cc}
0 & -\frac{R_{C}}{L} \\
0 & 0
\end{array}\right] \cdot \mathbf{w} \cdot u \\
& y=\left[R_{C} \cdot(1-D) 1\right] \cdot\left[\begin{array}{c}
i_{L} \\
v_{C}
\end{array}\right]+\left[\begin{array}{ll}
0 & -R_{C}
\end{array}\right] \cdot\left[\begin{array}{l}
v_{i n} \\
i_{o}
\end{array}\right]=\mathbf{C} \cdot \mathbf{x}+\mathbf{E}_{\mathbf{y}} \cdot \mathbf{w}
\end{aligned}
$$

If we neglect the parasitics, 


$$
\begin{gathered}
\mathbf{X}_{\mathbf{0} l}=\left[\begin{array}{c}
\frac{1}{1-D} \cdot I_{o} \\
\frac{1}{1-D} \cdot V_{i n}
\end{array}\right] \\
\dot{\mathbf{x}}=\left[\begin{array}{cc}
0 & -\frac{1-D}{L} \\
\frac{1-D}{C} & 0
\end{array}\right] \cdot \mathbf{x}+\left[\begin{array}{c}
\frac{V_{i n}}{L \cdot(1-D)} \\
-\frac{I_{o}}{C \cdot(1-D)}
\end{array}\right] \cdot u+\left[\begin{array}{c}
\frac{1}{L} \\
0
\end{array}\right] \cdot v_{i n}+\left[\begin{array}{c}
0 \\
-\frac{1}{C}
\end{array}\right] \cdot i_{o}+\left[\begin{array}{cc}
0 & \frac{1}{L} \\
-\frac{1}{C} & 0
\end{array}\right] \cdot \mathbf{x} \cdot u= \\
=\left[\begin{array}{cc}
0 & -\frac{1-D}{L} \\
\frac{1-D}{C} & 0
\end{array}\right] \cdot \mathbf{x}+\left[\begin{array}{c}
\frac{V_{i n}}{L \cdot(1-D)} \\
-\frac{I_{o}}{C \cdot(1-D)}
\end{array}\right] \cdot u+\left[\begin{array}{cc}
\frac{1}{L} & 0 \\
0 & -\frac{1}{C}
\end{array}\right] \cdot \mathbf{w}+\left[\begin{array}{cc}
0 & \frac{1}{L} \\
-\frac{1}{C} & 0
\end{array}\right] \cdot \mathbf{x} \cdot u \\
y=\left[\begin{array}{ll}
0 & 1
\end{array}\right] \cdot \mathbf{x}=\mathbf{C} \cdot \mathbf{x}
\end{gathered}
$$

A Resistive Load (RL), $R_{l d}$, becomes part of the passive structure of the electrical network during each cycle and therefore the model for each half cycle changes by replacing $i_{o}=v_{o} / R_{l d}$ as a function of the states and the rest of the passive elements.

$$
i_{o}=\frac{v_{o}}{R_{l d}}=\left\{\begin{array}{l}
\frac{x_{2}}{R_{C}+R_{l d}}, \quad \mathrm{~S}-\mathrm{ON} \\
\frac{x_{2}+R_{C} \cdot x_{1}}{R_{C}+R_{l d}}, \quad \mathrm{~S}-\mathrm{OFF}
\end{array} .\right.
$$

Substituting (2.2-26) in (2.2-20) and (2.2-21) renders the same model obtained by the existing methods and described in (2.1-5), (2.1-6). If there are no parasitics,

$$
\begin{gathered}
\mathbf{X}_{\mathbf{0} r l}=\left[\begin{array}{c}
\frac{1}{R_{l d} \cdot(1-D)^{4}} \cdot V_{i n} \\
\frac{1}{1-D} \cdot V_{i n}
\end{array}\right] \\
\dot{\mathbf{x}}=\left[\begin{array}{cc}
0 & -\frac{(1-D)}{L} \\
\frac{(1-D)}{C} & -\frac{1}{R_{l d} \cdot C}
\end{array}\right] \cdot \mathbf{x}+\left[\begin{array}{c}
\frac{V_{i n} \cdot \frac{1}{1-D}}{1-V_{i n} \cdot \frac{1}{(1-D)^{2}} \cdot \frac{1}{R_{l d} \cdot C}}
\end{array}\right] \cdot u+\left[\begin{array}{c}
\frac{1}{L} \\
0
\end{array}\right] \cdot v_{i n}+\left[\begin{array}{cc}
0 & \frac{1}{L} \\
-\frac{1}{C} & 0
\end{array}\right] \cdot \mathbf{x} \cdot u \\
y=\left[\begin{array}{ll}
0 & 1
\end{array}\right] \cdot \mathbf{x}
\end{gathered}
$$




\subsection{Resistive Load variations as a Robustness problem}

\subsubsection{A Clarification for the Existing Small Signal Models}

Equation (2.2-28) shows now much more clearly the resistive load robustness and disturbance rejection issues. Any change in the $R_{l d}$, load, e.g., a load step, will appear as a change in the internal terms of the plant model matrices, hence a robustness problem. For large perturbations the state space models should consider not only the state perturbations but also the new load resistances, e.g., $R_{l d \_n o m} \| R_{l d \_s t e p}$ in the case of a load step, for example.

For very low perturbations, the modification in the $\mathbf{A}$ and $\mathbf{B}$ matrices terms can be neglected and hence the present linear models can be successfully used for the simplified linear analysis, even though they do not incorporate a fully correct structure; this clarification is needed for the consistency of all models employed in DC/DC converters analysis.

\subsubsection{Structure Change Quantification}

Equation (2.2-28) also shows that the load resistance only impacts the regulator significantly through the $a_{22}$ and the $b_{2}$ terms, of the respective $\mathbf{A}$ and $\mathbf{B}$ matrices. When a step load occurs, the new load resistance is the parallel combination of the nominal and the step load one,

$$
\frac{1}{R_{l d}}=\frac{1}{R_{l d_{-} \text {nom }}}+\frac{1}{R_{l d_{\_} \text {step }}}
$$

and therefore the converter's model becomes

$$
\begin{aligned}
& \dot{\mathbf{x}}=\left(\left[\begin{array}{cc}
0 & -\frac{(1-D)}{L} \\
\frac{(1-D)}{C} & -\frac{1}{C} \cdot \frac{1}{R_{\text {ld_ nom }}}
\end{array}\right]+\left[\begin{array}{cc}
0 & 0 \\
0 & -\frac{1}{C} \cdot \frac{1}{R_{\text {ld_ } \_ \text {по }}}
\end{array}\right] \cdot \frac{1}{x t p} \cdot \operatorname{Step}\left(t-t_{\text {step }}\right)\right) \cdot \mathbf{x}+
\end{aligned}
$$

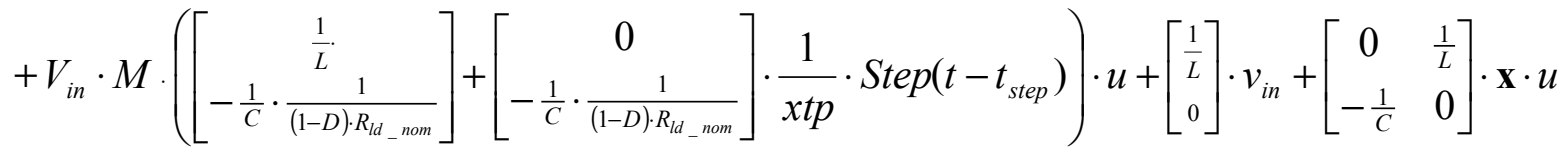


with $x t p=R_{l d \_s t e p} / R_{l d \_n o m}$, the percentage of the load step occurring at $t_{\text {step }}$ and $M$ the dc voltage conversion ratio. Equation (2.3-2) quantifies how the robustness problem comes in for the resistive load case, by re-writing it in the compact form

$$
\dot{\mathbf{x}}=(\mathbf{A}+\mathbf{L}) \cdot \mathbf{x}+(\mathbf{B}+\mathbf{L}) \cdot u+\mathbf{E}^{\mathbf{v}_{\text {in }}} \cdot v_{i n}+\Delta \mathbf{A} \cdot \mathbf{x} \cdot u
$$

Equation (2.3-4) shows also why it is not easy to capture this modeling form when simulating the circuit in Matlab. This is because one can not step elements inside the matrices, themselves, in the existing software packages. By using the unterminated model in this new form, one has now an unified, straightforward approach to model the system from a modern control formalism standpoint.

Since the main objective of this study is improved current sharing for master-slave paralleled converters, only the load disturbance effects will be studied in detail. Solving the problem of output (disturbance) current sharing is of higher importance and more difficult due to its way of coming into the plant's structure. The input voltage comes in linearly and independently, hence its study is more straight forward and can be considered for future research. We obtain thus the bilinear form

$$
\dot{\mathbf{x}}=\mathbf{A} \cdot \mathbf{x}+\mathbf{B} \cdot u+\Delta \mathbf{A} \cdot \mathbf{x} \cdot u
$$

All basic DC/DC converter topologies can be factorized in this manner, by containing the $1 / R_{l d}$ term, which in the loss-less case appears only in the last, main diagonal, element of the $\mathbf{A}$ matrix and, sometimes, in the $\mathbf{B}$ vector. This shows the importance of separating the real converter structure from the case including the parasitics in order to properly highlight the control problem.

The form (2.3-4) will be employed for the new control law analysis and stability design while forms (2.2-20), (2.2-21) will be used for verifying the design, in the simulated results, which are run with a full converter model that includes parasitics, saturations, etc.. 


\subsection{Constant Power Load Case}

\subsubsection{Constant Power Load as an independent source}

For a constant power load (CPL), $i_{o}=-p_{l d} / v_{o}$ ac-wise independently of the network passive elements and for either position of the switch, since the constant power source is an independent, exogenous source. We obtain

$$
\mathbf{X}_{\mathbf{0} p}=\left[\begin{array}{c}
\frac{1}{1-D} \cdot \frac{P_{l d 0}}{Y_{0}} \\
\frac{1}{1-D} \cdot V_{i n}-\left[\frac{D}{1-D} \cdot R_{C}+\frac{R_{L}}{(1-D)^{2}}\right] \cdot \frac{P_{l d}}{Y_{0}}
\end{array}\right]
$$

and

$$
\begin{aligned}
\dot{\mathbf{x}} & =\left[\begin{array}{cc}
-\frac{R_{l}+R_{C} \cdot(1-D)}{L} & -\frac{1-D}{L} \\
\frac{1-D}{C} & 0
\end{array}\right] \cdot \mathbf{x}+\left[\begin{array}{c}
\frac{V_{i n} \cdot(1-D)-\frac{P_{l d}}{Y_{o}} \cdot R_{l}}{L \cdot(1-D)^{2}} \\
-\frac{1}{C \cdot(1-D)} \cdot \frac{P_{l d}}{Y_{o}}
\end{array}\right] \cdot u+\left[\begin{array}{c}
\frac{1}{L} \\
0
\end{array}\right] \cdot v_{i n}+\left[\begin{array}{c}
\frac{R_{C} \cdot(1-D)}{L} \\
\frac{1}{C}
\end{array}\right] \cdot \frac{p_{l d}}{y}+\left[\begin{array}{cc}
\frac{R_{C}}{L} & \frac{1}{L} \\
-\frac{1}{C} & 0
\end{array}\right] \cdot \mathbf{x} \cdot u+ \\
& +\left[\begin{array}{c}
\frac{R_{C}}{L} \\
0
\end{array}\right] \cdot \frac{p_{l d}}{y} \cdot u \\
y & =\left[\begin{array}{ll}
R_{C} \cdot(1-D) & 1
\end{array}\right] \cdot \mathbf{x}+\left[\begin{array}{ll}
0 & R_{C}
\end{array}\right] \cdot\left[\begin{array}{c}
v_{i n} \\
\frac{p_{l d}}{y}
\end{array}\right]
\end{aligned}
$$

If we have no parasitics,

$$
\begin{aligned}
\dot{\mathbf{x}} & =\left[\begin{array}{cc}
0 & -\frac{1-D}{L} \\
\frac{1-D}{C} & 0
\end{array}\right] \cdot \mathbf{x}+\left[\begin{array}{c}
\frac{V_{i n}}{L \cdot(1-D)} \\
-\frac{1}{C \cdot(1-D)} \cdot \frac{P_{l d}}{Y_{o}}
\end{array}\right] \cdot u+\left[\begin{array}{c}
\frac{1}{L} \\
0
\end{array}\right] \cdot v_{i n}+\left[\begin{array}{c}
0 \\
\frac{1}{C}
\end{array}\right] \cdot \frac{p_{l d}}{y}+\left[\begin{array}{cc}
0 & \frac{1}{L} \\
-\frac{1}{C} & 0
\end{array}\right] \cdot \mathbf{x} \cdot u= \\
& =\mathbf{A}_{p l} \cdot \mathbf{x}+\mathbf{B}_{p l} \cdot u+\mathbf{E}_{\mathbf{x} p l}^{\mathbf{v}_{\text {in }}} \cdot v_{i n}+\mathbf{E}_{\mathbf{x} p l}^{\mathbf{i}_{\mathrm{o}}} \cdot \frac{p_{l d}}{y}+\Delta \mathbf{A}_{p l} \cdot \mathbf{x} \cdot u \\
y & =\left[\begin{array}{ll}
0 & 1
\end{array}\right] \cdot \mathbf{x}=\mathbf{C} \cdot \mathbf{x}
\end{aligned}
$$

It can be seen that besides the nonlinearity, the CPL has the potential to destabilize the system by introducing RHPs through its negative resistance behavior, as it will be shown in the next paragraph. 


\subsubsection{Constant Power load as an equivalent negative resistance}

It is useful to also write the converter's model with a negative load resistance equivalent to the CPL. The negative incremental resistance behavior was often explicated in the literature and can be easily seen by writing the expression of the load power as the product of current and voltage, acquaint for the signs (when the voltage increases the current decreases and vice-versa) and then express one of the quantities as a function of the equivalent load resistance. Now, one can use (2.4-3) with $R_{l d}$ replaced with $-R_{C P L}$ and similarly with (2.3-2), we have that

$$
\begin{aligned}
& \dot{\mathbf{x}}=\left(\left[\begin{array}{cc}
0 & -\frac{(1-D)}{L} \\
\frac{(1-D)}{C} & \frac{1}{C} \cdot \frac{1}{R_{\text {CPL_nom }}}
\end{array}\right]+\left[\begin{array}{cc}
0 & 0 \\
0 & \frac{1}{C} \cdot \frac{1}{R_{\text {CPL_nom }}}
\end{array}\right] \cdot \frac{1}{x t p} \cdot \operatorname{Step}\left(t-t_{\text {step }}\right)\right) \cdot \mathbf{x}+ \\
& +V_{\text {in }} \cdot M \cdot\left(\left[\begin{array}{c}
\frac{1}{L} \\
\frac{1}{C} \cdot \frac{1}{(1-D) \cdot R_{\text {CPL_nom }}}
\end{array}\right]+\left[\begin{array}{c}
0 \\
\left.\frac{1}{C} \cdot \frac{1}{(1-D) \cdot R_{\text {CPL_nom }}}\right]
\end{array}\right] \cdot \frac{1}{x t p} \cdot \operatorname{Step}\left(t-t_{\text {step }}\right)\right) \cdot u+\left[\begin{array}{c}
\frac{1}{L} \\
0
\end{array}\right] \cdot v_{\text {in }}+\left[\begin{array}{cc}
0 & \frac{1}{L} \\
-\frac{1}{C} & 0
\end{array}\right] \cdot \mathbf{x} \cdot u
\end{aligned}
$$

Several studies have been carried out to deal with the potential instability introduced by the negative resistance equivalent with the constant power load, most of them with practical results. In practice, constant power loads have a resistive behavior for low voltages and thus a positive load resistance, of very high value, exists in parallel with the CPL; around the steady state operating point, the negative resistance behavior takes place. Theoretically, for any electrical network that gets energized, if a perfectly equivalent, but negative impedance is placed in tandem with that network's positive output impedance, then oscillations will occur. Practically, depending of how large the CPL perturbation is, the system may still be stable provided there is enough damping (positive resistance) at the respective interface for that $\mathrm{DC}$ operating point. In those cases, using the unterminated model and applying the Nyquist criterion, one can still have a stable system, even though RHP poles exist, [31]. Each example is analyzed on an application specific basis and several means of compensating the current sharing loop have been used. The studies in [32], [33], specify the output impedance and vary the DC gain of a simple PD controller, in order to "schedule" the gain for each operating point. In other 
cases, the stability task is carried out just by reducing the bandwidth of the classical PI OpAmp controllers and making sure that no large negative resistance CPLs occur.

Another, successful scheme is to feed back the information about the load current level and then employ the calculation of the output and input powers in order to make the DC gain of the control to output function practically invariant with the load [17]. This approach offers a performant and more stable large signal response in the case of a CPL, since it practically zeros out the output impedance of that converter.

The objective of this study is to introduce and develop a fuzzy logic controller for the current sharing compensator, which, just by itself requires substantial detailing and analysis. Therefore, all the subsequent work will be done considering the resistive load case. For a different load type, such as CPL, the new control law would have to be appended with additional constraints so the negative resistance behavior is compensated within the feasibility limits of each application. This is listed as one of the suggestions for future research since the load type does not invalidate the idea of employing new control strategies using emerging fuzzy techniques, which is the main focus here. They may need to be adapted when considering the load type, but they need to be first created and developed in their basic form so they can be extended, next, as part of a different study. 


\subsection{Closed Loop Model}

By replacing the control input, $u$, with its expression as a function of the state feedback variables, a closed loop model is obtained. As an illustration, the most used control strategy today for PWM, i.e., Peak Current Mode Control (PCMC), converters will be used, because it offers a general, unified approach; the expressions for Voltage Mode Control (VMC) and Average Current Mode Control (ACMC) can be written in the same form for the feedback function, less and respectively more complicated, but without any major difference in what concerns the goal and final outcome of this study.

Small signal models including the sample and hold effect, " $H_{e}(s)$ ", [6], have been currently developed, also in a modern, state space, formalism, [7]. Since in this study we are addressing the nonlinear, fuzzy logic control of the output current sharing of master - slave paralleled converter systems under large load disturbance, we are mainly interested in the system's behavior from a large signal point of view. Moreover, in dealing with a paralleled system, one assumes that the individual DC/DC regulator modules are already properly designed in terms of their own small signal stability and hence $H_{e}(s)$ was employed, before they are integrated in the respective distributed system. Therefore, in order to concentrate on the new control approach, without restricting any generality, we will assume that the module design has the appropriate ramp compensation and the effect of $H_{e}(s)$ are negligible, especially at a system level. The equations can be adapted to include the detailed sample and hold model, but they would only become cumbersome to read in state space form, with no real contribution to the newly proposed fuzzy logic control for the current sharing loop.

Based on the above discussions, consider again the general loss-less model of the boost converter,

$$
\begin{gathered}
\dot{\mathbf{x}}=\left[\begin{array}{cc}
0 & -\frac{(1-D)}{L} \\
\frac{(1-D)}{C} & -\frac{1}{R_{l d} \cdot C}
\end{array}\right] \cdot \mathbf{x}+V_{i n} \cdot M \cdot\left[\begin{array}{c}
\frac{1}{L} \\
-\cdot \frac{1}{(1-D)} \cdot \frac{1}{R_{l d} \cdot C}
\end{array}\right] \cdot u+\left[\begin{array}{cc}
0 & \frac{1}{L} \\
-\frac{1}{C} & 0
\end{array}\right] \cdot \mathbf{x} \cdot u= \\
y=\mathbf{C} \cdot \mathbf{x} \\
\dot{\mathbf{x}}=\mathbf{A} \cdot \mathbf{x}+\mathbf{B} \cdot u+\Delta \mathbf{A} \cdot \mathbf{x} \cdot u,
\end{gathered}
$$

or 
For a complete record, if the input voltage perturbation were also considered and an unterminated model were used instead of including the load impedance in the plant structure, we would have that

$$
\dot{\mathbf{x}}=\mathbf{A} \cdot \mathbf{x}+\mathbf{B} \cdot u+\mathbf{E} \cdot \mathbf{w}+\Delta \mathbf{A} \cdot \mathbf{x} \cdot u+\Delta \mathbf{E} \cdot \mathbf{w} \cdot u
$$

In addition, especially for large signal paralleled DC-DC systems there is no real advantage in separating the converter circuit at the modulator output in order to consider the real modulator circuit and use the modulator's gain expression that comes out from the signal theory for that circuit. That is what the existing modeling techniques do so they can use in their calculations the quantities that are available in the converter circuitry. One of the main reasons behind it is to be able to measure for each individual module, on the real hardware, the associated gains, in this case proportional with the inverse of the PWM reference ramp amplitude. However, the integrated box level results are practically identical if the mathematical averaged model is used as a whole, for the overall converter module. That is how all the models start, but they are then split and get modified at the PWM modulator output. Reference [7] comes the closest to addressing this issue, highlighting both forms, but with a different goal, i.e., trying to include the effects of " $H_{e}(s)$ " in a state space form. Those forms are useful in the single converter small signal, Bode analysis, but they would just hide the real control issues revealed here, when used for the paralleled system analysis.

Based on the above findings, considering the overall averaged model and neglecting the high frequency effects - $H_{e}(s)$, the PCMC control input can be written as, [7], [10], [18],

$$
u=F_{m} \cdot v_{e a}-\mathbf{F}_{\mathrm{ix}} \cdot \mathbf{x}-\mathbf{F}_{\mathrm{iw}} \cdot \mathbf{w}
$$

if the unterminated model were used, with

$$
\begin{aligned}
& \mathbf{F}_{\mathrm{ix}}=\mathbf{C}_{\mathbf{i}} \cdot\left(\mathbf{I}+\frac{D \cdot T_{s}}{2} \cdot \mathbf{A}_{\mathbf{1}}\right), \\
& \mathbf{F}_{\mathrm{iw}}=\frac{D \cdot T_{s}}{2} \cdot \mathbf{C}_{\mathbf{i}} \cdot \mathbf{E}_{1 \mathbf{x}}=\left[\begin{array}{ll}
\mathbf{F}_{\mathbf{i}}^{\mathbf{v}_{\text {in }}} & \mathbf{F}_{\mathbf{i}}^{\mathbf{i}_{\mathbf{o}}}
\end{array}\right]=\left[\begin{array}{ll}
\mathbf{F}_{\mathbf{i}}^{\mathbf{v}_{\text {in }}} & \mathbf{0}
\end{array}\right],
\end{aligned}
$$


since $\mathbf{C}_{\mathbf{i}}=\left[\begin{array}{ll}\mathrm{R}_{\mathrm{i}} & 0\end{array}\right], \mathrm{R}_{\mathrm{i}}$ being the equivalent resistance and ratio of the current sensing network.

If the model with zero input voltage perturbation (as in this study) and the load resistance incorporated were used, we would have

$$
u=F_{m} \cdot v_{e a}-\mathbf{F}_{\mathbf{i x}} \cdot \mathbf{x}
$$

The unterminated model will be used mainly in simulation and the detailed model, with the load impedance incorporated, will be used mainly for stability issues in the control systems design.

The main (voltage) loop compensator is described by

$$
\begin{aligned}
& \dot{\mathbf{x}}_{\mathbf{f}}=\mathbf{A}_{\mathbf{f}} \cdot \mathbf{x}_{\mathbf{f}}+\mathbf{B}_{\mathbf{f}} \cdot\left(v_{r e f}-x_{2}\right)=\mathbf{A}_{\mathbf{f}} \cdot \mathbf{x}_{\mathbf{f}}+\left[\begin{array}{ll}
0 & -\mathbf{B}_{\mathbf{f}}
\end{array}\right] \cdot \mathbf{x}+\mathbf{B}_{\mathbf{f}} \cdot v_{r e f} \\
& v_{e a}=\mathbf{C}_{\mathbf{f}} \cdot \mathbf{x}_{\mathbf{f}}+\mathbf{D}_{\mathbf{f}} \cdot\left(v_{r e f}-x_{2}\right)+v_{r e f}=\mathbf{C}_{\mathbf{f}} \cdot \mathbf{x}_{\mathbf{f}}+\left[\begin{array}{ll}
0 & -\mathbf{D}_{\mathbf{f}}
\end{array}\right] \cdot \mathbf{x}+\left(\mathbf{D}_{\mathbf{f}}+\mathbf{I}\right) \cdot v_{r e f}
\end{aligned} .
$$

This comes from the TF description of the OpAmp, having $v_{r e f}$ at its non-inverting input and $x_{2}$ at its inverting one,

$$
V_{e a}(s)=\left[1+H_{v}(s)\right] \cdot V_{r e f}(s)-H_{v}(s) \cdot X_{2}(s)=H_{v}(s) \cdot\left[V_{r e f}(s)-X_{2}(s)\right]+V_{r e f}(s),
$$

where the output divider ration, $k_{d}$, is assumed incorporated into the $x_{2}$ quantity.

For example, for a "type 2" compensator employed in PCMC and given in Figure 2.5-1 below,

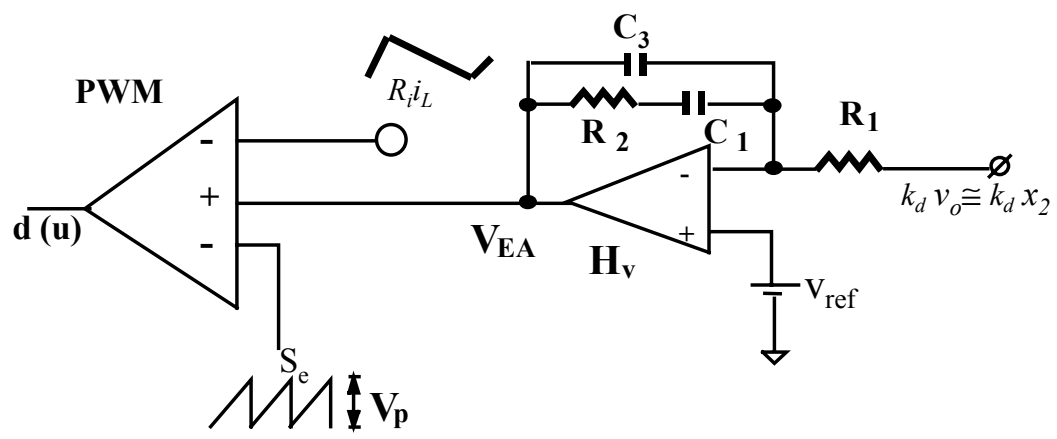

Figure 2.5-1 - PCMC Control Scheme 


$$
\begin{aligned}
H_{v}(s) & =\frac{V_{e a}(s)}{-k_{d} \cdot X_{2}(s)}=\frac{1}{s \cdot R_{1} \cdot\left(C_{1}+C_{3}\right)} \cdot \frac{\left(1+\frac{s}{\frac{1}{R_{2} \cdot C_{1}}}\right)}{\left(1+\frac{s}{\frac{1}{R_{2} \cdot \frac{C_{1} \cdot C_{3}}{C_{1}+C_{3}}}}\right)}=\frac{\omega_{i 1} \cdot \frac{\omega_{z 1}}{\omega_{p 1}} \cdot\left(1+\frac{s}{\omega_{z 1}}\right)}{s}= \\
= & \frac{1}{s \cdot R_{1} \cdot C_{3}} \cdot \frac{\left(1+\frac{s}{\omega_{p 1}}\right)}{\left(s+\frac{1}{R_{2} \cdot C_{1}}\right)}=\frac{\omega_{i}}{s} \cdot \frac{\left(s+\omega_{z 1}\right)}{\left(s+\omega_{p 1}\right)}=\frac{\omega_{i}}{s} \cdot\left(1+\frac{\omega_{z 1}-\omega_{p 1}}{s+\omega_{p 1}}\right)
\end{aligned}
$$

and hence the matrices in (2.5-8) are

$$
\mathbf{A}_{\mathbf{f}}=\left[\begin{array}{cc}
-\omega_{p 1} & 0 \\
\omega_{z 1}-\omega_{p 1} & 0
\end{array}\right], \quad \mathbf{B}_{\mathbf{f}}=\left[\begin{array}{l}
1 \\
1
\end{array}\right], \quad \mathbf{C}_{\mathbf{f}}=\omega_{i} \cdot\left[\begin{array}{ll}
0 & 1
\end{array}\right], \quad \mathbf{D}_{\mathbf{f}}=[0] .
$$

For a "type 3" compensator, used in VMC, the structure would be similar, but of third order. A block diagram of the new large signal model for the PCMC DC/DC Regulator is given in Figure 2.5-2 below

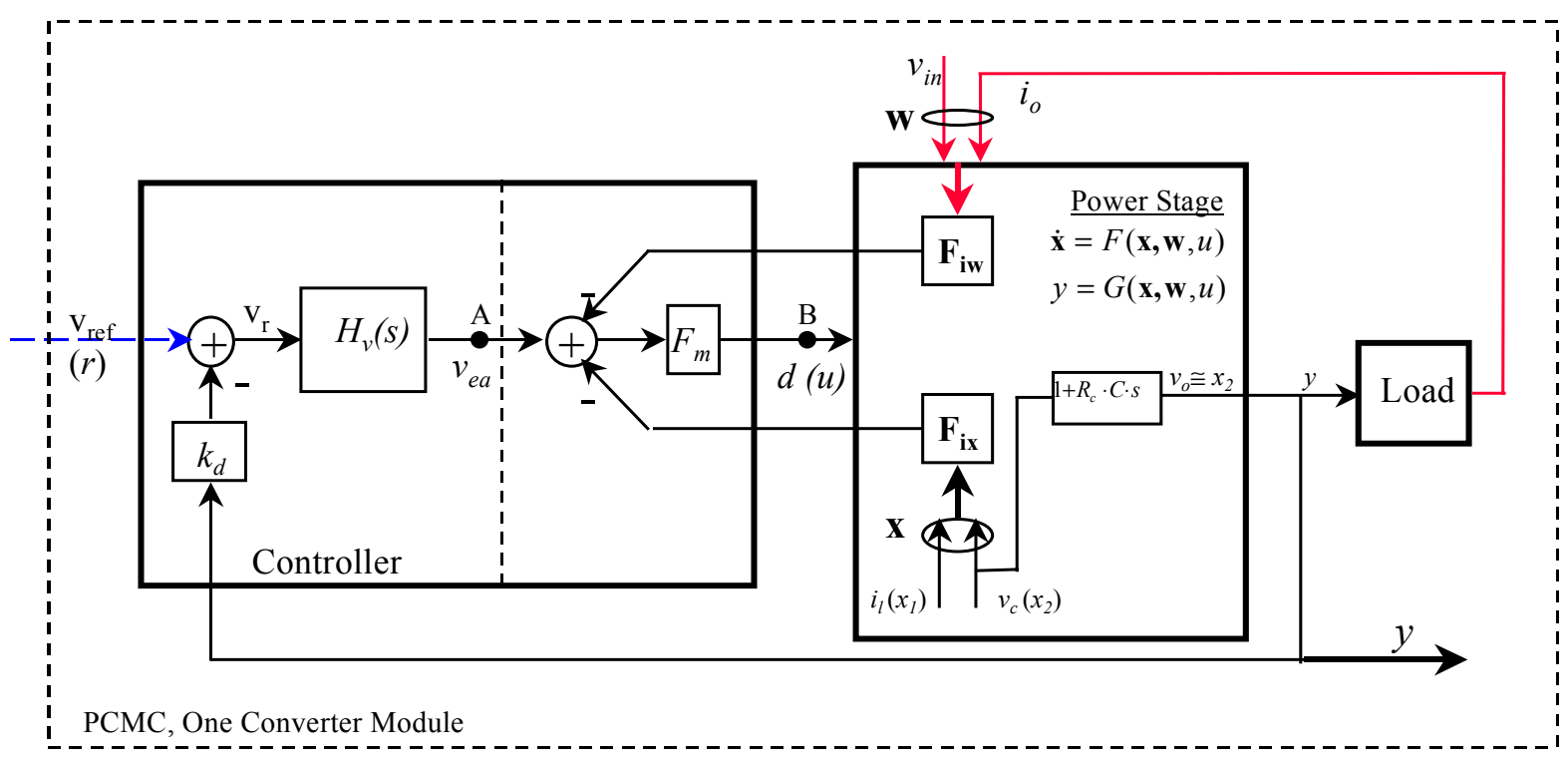

Figure 2.5-2 - PCMC DC/DC Regulator Control Block diagram

It can be seen now that following this approach, the converter can be modeled in a straightforward, explicit way in simulators like Simulink, for subsequent design developments. 
Using (2.5-7) in (2.5-2) gives

$$
\dot{\mathbf{x}}=\left(\mathbf{A}-F_{m} \cdot \mathbf{B} \cdot \mathbf{F}_{\mathrm{ix}}\right) \cdot \mathbf{x}-F_{m} \cdot \Delta \mathbf{A} \cdot \mathbf{x} \cdot \mathbf{F}_{\mathrm{ix}} \cdot \mathbf{x}+(\mathbf{B}+\Delta \mathbf{A} \cdot \mathbf{x}) \cdot\left(F_{m} \cdot v_{e a}\right)
$$

or, on short,

$$
\dot{\mathbf{x}}=f_{1}(\mathbf{x})+g_{1}(\mathbf{x}) \cdot\left(F_{m} \cdot v_{e a}\right)
$$

Using (2.5-8) in (2.5-13) gives the augmented plant, i.e., closed loop model

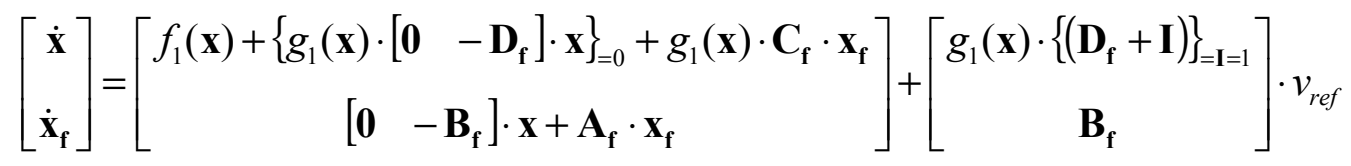

If we want to detail the terms so we depict each matrix involved, we obtain

$$
\begin{aligned}
& {\left[\begin{array}{c}
\dot{\mathbf{x}} \\
\dot{\mathbf{x}}
\end{array}\right]=\left[\begin{array}{c|c}
\mathbf{A}-F_{m} \cdot \mathbf{B} \cdot \mathbf{C}_{\mathbf{i}} \cdot\left(\mathbf{I}+\frac{D \cdot T_{s}}{2} \cdot \mathbf{A}_{1}\right)-F_{m} \cdot \Delta \mathbf{A} \cdot \mathbf{x} \cdot \mathbf{C}_{\mathbf{i}} \cdot\left(\mathbf{I}+\frac{D \cdot T_{s}}{2} \cdot \mathbf{A}_{\mathbf{1}}\right) & F_{m} \cdot(\mathbf{B}+\Delta \mathbf{A} \cdot \mathbf{x}) \cdot \mathbf{C}_{\mathbf{f}} \\
\hline\left[\begin{array}{l}
\mathbf{0}-\mathbf{B}_{\mathbf{f}}
\end{array}\right]
\end{array}\right] \cdot\left[\begin{array}{c}
\mathbf{x} \\
\mathbf{x}_{\mathbf{f}}
\end{array}\right]+}
\end{aligned}
$$

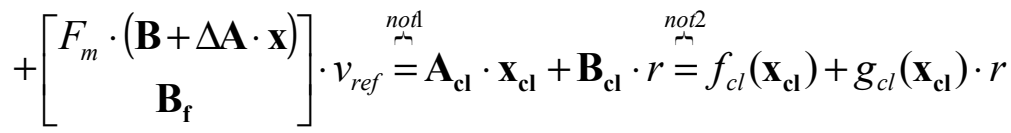

Expanding the terms for the boost converters we obtain the large signal model

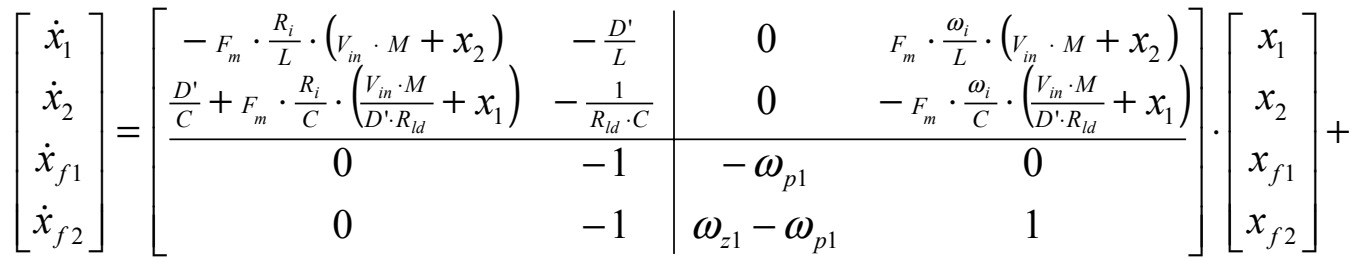

$$
\begin{aligned}
& +\left[\begin{array}{c}
F_{m} \cdot \frac{1}{L} \cdot\left(V_{\text {in }} \cdot M+x_{2}\right) \\
-F_{m} \cdot \frac{1}{C} \cdot\left(\frac{V_{i n} \cdot M}{D^{\prime} \cdot R_{l d}}+x_{1}\right) \\
1 \\
1
\end{array}\right] \cdot v_{r e f}
\end{aligned}
$$

or, in greater detail, 


$$
\begin{aligned}
& {\left[\begin{array}{c}
\dot{x}_{1} \\
\dot{x}_{2} \\
\dot{x}_{f 1} \\
\dot{x}_{f 2}
\end{array}\right]=} {\left[\begin{array}{c}
-F_{m} \cdot V_{i n} \cdot M \cdot \frac{R_{i}}{L} \cdot x_{1}-F_{m} \cdot \frac{R_{i}}{L} \cdot x_{1} \cdot x_{2}-\frac{D^{\prime}}{L} \cdot x_{2}+F_{m} \cdot \frac{\omega_{i}}{L} \cdot V_{i n} \cdot M \cdot x_{f 2}+F_{m} \cdot \frac{\omega_{i}}{L} \cdot x_{2} \cdot x_{f 2} \\
\left(\frac{D^{\prime}}{C}+F_{m} \cdot V_{i n} \cdot M \cdot \frac{R_{i}}{D^{\prime} \cdot R_{l d} \cdot C}\right) \cdot x_{1}+F_{m} \cdot \frac{R_{i}}{C} \cdot x_{1}^{2}-\frac{1}{R_{l d} \cdot C} \cdot x_{2}-F_{m} \cdot V_{i n} \cdot M \cdot \frac{\omega_{i}}{D^{\prime} \cdot R_{l d} \cdot C} \cdot x_{f 2}-F_{m} \cdot \frac{\omega_{i}}{C} \cdot x_{1} \cdot x_{f 2} \\
-x_{2}-\omega_{p 1} \cdot x_{f 1} \\
-x_{2}+\left(\omega_{z 1}-\omega_{p 1}\right) \cdot x_{f 1}+x_{f 2}
\end{array}\right]+} \\
&+\left[\begin{array}{c}
F_{m} \cdot \frac{1}{L} \cdot\left(\begin{array}{c}
V_{i n} \cdot M \\
-F_{m} \cdot \frac{1}{C} \cdot\left(\frac{V_{i n} \cdot M}{D^{\prime} \cdot R_{l d}}+x_{1}\right) \\
1
\end{array}\right] \cdot v_{r e f}
\end{array}\right] .
\end{aligned}
$$

For the buck converter, we obtain

$$
\left[\begin{array}{c}
\dot{x}_{1} \\
\dot{x}_{2} \\
\dot{x}_{f 1} \\
\dot{x}_{f 2}
\end{array}\right]=\left[\begin{array}{cc|cc}
-F_{m} \cdot \frac{R_{i}}{L} \cdot V_{i n} & \frac{1}{L} \cdot\left(F_{m} \cdot \frac{R_{i}}{L} \cdot V_{i n} \cdot \frac{D \cdot T_{s}}{2}-1\right) & 0 & F_{m} \cdot \frac{\omega_{i}}{L} \cdot V_{i n} \\
\frac{1}{C} & -\frac{1}{R_{l d} \cdot C} & 0 & 0 \\
\hline 0 & -1 & -\omega_{p 1} & 0 \\
0 & -1 & \omega_{z 1}-\omega_{p 1} & 1
\end{array}\right] \cdot\left[\begin{array}{c}
x_{1} \\
x_{2} \\
x_{f 1} \\
x_{f 2}
\end{array}\right]+\left[\begin{array}{c}
F_{m} \cdot \frac{1}{L} \cdot V_{i n} \\
0 \\
1 \\
1
\end{array}\right] \cdot v_{r e f} \cdot
$$

As known, the buck converter's model is linear, if no input voltage perturbation is considered. If one would like to keep the unterminated model form and also include the input voltage perturbation and parasitics, we obtain by using (2.5-3) and (2.5-4)

$$
\begin{aligned}
& \dot{\mathbf{x}}=\underbrace{\left(\mathbf{A}-F_{m} \cdot \mathbf{B} \cdot \mathbf{F}_{\mathbf{i x}}\right) \cdot \mathbf{x}-F_{m} \cdot \Delta \mathbf{A} \cdot \mathbf{x} \cdot \mathbf{F}_{\mathbf{i x}} \cdot \mathbf{x}}_{f_{1}(\mathbf{x})}+\underbrace{F_{m} \cdot(\mathbf{B}+\Delta \mathbf{A} \cdot \mathbf{x})}_{g_{1}(\mathbf{x})} \cdot v_{e a}+\underbrace{F_{m} \cdot \Delta \mathbf{E}_{\mathbf{x}} \cdot \mathbf{W}}_{q_{1}(\mathbf{w})} \cdot v_{e a} \\
& +\underbrace{\left(\mathbf{E}_{\mathbf{x}}-F_{m} \cdot \Delta \mathbf{A} \cdot \mathbf{x} \cdot \mathbf{F}_{\mathrm{iw}}-F_{m} \cdot \mathbf{B} \cdot \mathbf{F}_{\mathrm{iw}}\right)}_{p_{1}(\mathbf{x})} \cdot \mathbf{W}-\underbrace{F_{m} \cdot\left(\Delta \mathbf{E}_{\mathbf{x}} \cdot \mathbf{W} \cdot \mathbf{F}_{\mathrm{iw}} \cdot \mathbf{W}+\Delta \mathbf{E}_{\mathbf{x}} \cdot \mathbf{W} \cdot \mathbf{F}_{\mathrm{ix}} \cdot \mathbf{x}\right)}_{\mathbf{Q}_{\text {rest }}} \\
& y=\underbrace{\left(\mathbf{C}-F_{m} \cdot \mathbf{D} \cdot \mathbf{F}_{\mathbf{i x}}\right) \cdot \mathbf{x}-F_{m} \cdot \Delta \mathbf{C} \cdot \mathbf{x} \cdot \mathbf{F}_{\mathbf{i x}} \cdot \mathbf{x}}_{f_{2}(\mathbf{x})}+F_{m} \cdot \underbrace{(\mathbf{D}+\Delta \mathbf{C} \cdot \mathbf{x})}_{g_{2}(\mathbf{x})} \cdot v_{e a}+
\end{aligned}
$$

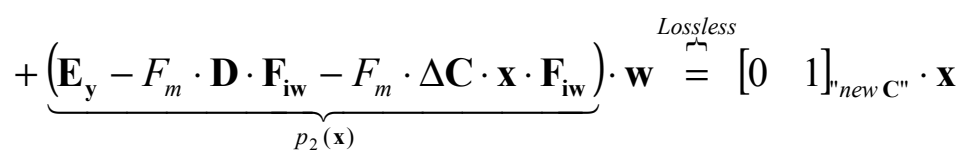

or, in a compact form,

$$
\dot{\mathbf{x}}=f_{1}(\mathbf{x})+\left(g_{1}(\mathbf{x})+q_{1}(\mathbf{w})\right) \cdot v_{e a}+p_{1}(x) \cdot \mathbf{w}+\mathbf{Q}_{\text {rest }}
$$




$$
y=f_{2}(\mathbf{x})+g_{2}(\mathbf{x}) \cdot v_{e a}+p_{2}(x) \cdot \mathbf{w} \stackrel{\text { Lossless }}{=} \mathbf{C} \cdot \mathbf{x}
$$

Detailing the terms so we depict each matrix involved, we obtain similarly to (2.5-15),

$$
\begin{aligned}
& {\left[\begin{array}{c}
\dot{\mathbf{x}} \\
\dot{\mathbf{x}}_{\mathbf{f}}
\end{array}\right]=\left[\begin{array}{cc}
\left(\mathbf{A}-F_{m} \cdot \mathbf{B} \cdot \mathbf{F}_{\mathbf{i x}}\right)-F_{m} \cdot \Delta \mathbf{A} \cdot \mathbf{x} \cdot \mathbf{F}_{\mathrm{ix}} & F_{m} \cdot\left(\mathbf{B}+\Delta \mathbf{A} \cdot \mathbf{x}+\Delta \mathbf{E}_{\mathbf{x}} \cdot \mathbf{w}\right) \cdot \mathbf{C}_{\mathbf{f}} \\
{[\mathbf{0}} & \left.-\mathbf{B}_{\mathbf{f}}\right]
\end{array}\right] \cdot\left[\begin{array}{c}
\mathbf{x} \\
\mathbf{x}_{\mathbf{f}}
\end{array}\right]+\left[\begin{array}{c}
\left(\mathbf{E}_{\mathbf{x}}-F_{m} \cdot \Delta \mathbf{A} \cdot \mathbf{x} \cdot \mathbf{F}_{\mathrm{iw}}-F_{m} \cdot \mathbf{B} \cdot \mathbf{F}_{\mathrm{iw}}\right) \\
\mathbf{0}
\end{array}\right] \cdot \mathbf{w}+} \\
& +\left[\begin{array}{c}
F_{m} \cdot\left(\mathbf{B}+\Delta \mathbf{A} \cdot \mathbf{x}+\Delta \mathbf{E}_{\mathbf{x}} \cdot \mathbf{w}\right) \\
\mathbf{B}_{\mathbf{f}}
\end{array}\right] \cdot v_{r e f}-\left[\begin{array}{c}
F_{m} \cdot\left(\Delta \mathbf{E}_{\mathbf{x}} \cdot \mathbf{w} \cdot \mathbf{F}_{\mathrm{iw}} \cdot \mathbf{w}+\Delta \mathbf{E}_{\mathbf{x}} \cdot \mathbf{W} \cdot \mathbf{F}_{\mathrm{ix}} \cdot \mathbf{x}\right) \\
\mathbf{0}
\end{array}\right] \\
& y=\left[\mathbf{C}-F_{m} \cdot \mathbf{D} \cdot \mathbf{F}_{\mathbf{i x}}-F_{m} \cdot \Delta \mathbf{C} \cdot \mathbf{x} \cdot \mathbf{F}_{\mathbf{i x}} \quad F_{m} \cdot(\mathbf{D}+\Delta \mathbf{C} \cdot \mathbf{x}) \cdot \mathbf{C}_{\mathbf{f}}\right] \cdot\left[\begin{array}{c}
\mathbf{x} \\
\mathbf{x}_{\mathbf{f}}
\end{array}\right]+ \\
& +\left(\mathbf{E}_{\mathbf{y}}-F_{m} \cdot \mathbf{D} \cdot \mathbf{F}_{\mathrm{iw}}-F_{m} \cdot \Delta \mathbf{C} \cdot \mathbf{x} \cdot \mathbf{F}_{\mathrm{iw}}\right) \cdot \mathbf{w}+F_{m} \cdot(\mathbf{D}+\Delta \mathbf{C} \cdot \mathbf{x}) \cdot v_{\text {ref }}^{\stackrel{\text { Lossless }}{=}} \mathbf{C} \cdot \mathbf{x}
\end{aligned}
$$

For the loss-less, zero input voltage perturbation model, equation (2.5-23) becomes

$$
\begin{aligned}
& {\left[\begin{array}{c}
\dot{\mathbf{x}} \\
\dot{\mathbf{x}}_{\mathbf{f}}
\end{array}\right]=\left[\begin{array}{c|c}
\left(\mathbf{A}-F_{m} \cdot \mathbf{B} \cdot \mathbf{F}_{\mathbf{i x}}\right)-F_{m} \cdot \Delta \mathbf{A} \cdot \mathbf{x} \cdot \mathbf{F}_{\mathbf{i x}} & F_{m} \cdot(\mathbf{B}+\Delta \mathbf{A} \cdot \mathbf{x}) \cdot \mathbf{C}_{\mathbf{f}} \\
{\left[\mathbf{0}-\mathbf{B}_{\mathbf{f}}\right]} & \mathbf{A}_{\mathbf{f}}
\end{array}\right] \cdot\left[\begin{array}{c}
\mathbf{x} \\
\mathbf{x}_{\mathbf{f}}
\end{array}\right]+\left[\begin{array}{c}
\mathbf{E}_{\mathbf{x}}^{\mathbf{i}_{0}} \\
\mathbf{0}
\end{array}\right] \cdot i_{o}+} \\
& +\left[\begin{array}{c}
F_{m} \cdot(\mathbf{B}+\Delta \mathbf{A} \cdot \mathbf{x}) \\
\mathbf{B}_{\mathbf{f}}
\end{array}\right] \cdot v_{r e f} \stackrel{n o t 1}{=} \mathbf{A}_{\mathbf{c l}} \cdot \mathbf{x}_{\mathbf{c l}}+\mathbf{E}_{\mathbf{c l}} \cdot w+\mathbf{B}_{\mathbf{c l}} \cdot r \stackrel{n o t 2}{=} f_{c l}\left(\mathbf{x}_{\mathbf{c l}}\right)+p_{c l}(\mathbf{x}) \cdot w+g_{c l}\left(\mathbf{x}_{\mathbf{c l}}\right) \cdot r
\end{aligned}
$$

These forms will be used in the next chapters to design the fuzzy logic control for the current sharing loop in the master-slave paralleled system. The complete model equations (2.5-23), (2.5-24) will be used in simulations and equation (2.5-15) will be mainly used for design and stability analysis. 


\subsubsection{Reference to Output Transfer Functions}

For and individual regulator module the terms in $v_{\text {ref }}$ are zero dynamics wise, e.g., [9] and all present small signal models derive their salient transfer functions based on this assumption. We will introduce and calculate the reference-to-output transfer function by considering the voltage reference term, since it is the control input for the paralleled system current sharing. In the slave converters, the current sharing compensator information is fed back through $v_{r e f}$, which in turns adjusts each converter output so its current tracks the one of the master.

Figure 2.5-3 shows the small signal diagram of a DC/DC converter, [9], which was appended with the reference voltage terms and a more detailed schematic of the OpAmp voltage loop compensator, explaining how the different terms are summed; also the feedforward and feedback gains $k_{f}$ and $k_{r}$, [6], are shown for reference, as they were not mentioned in [9]. Their values are small and can be neglected, for clarity, in the next derivations.

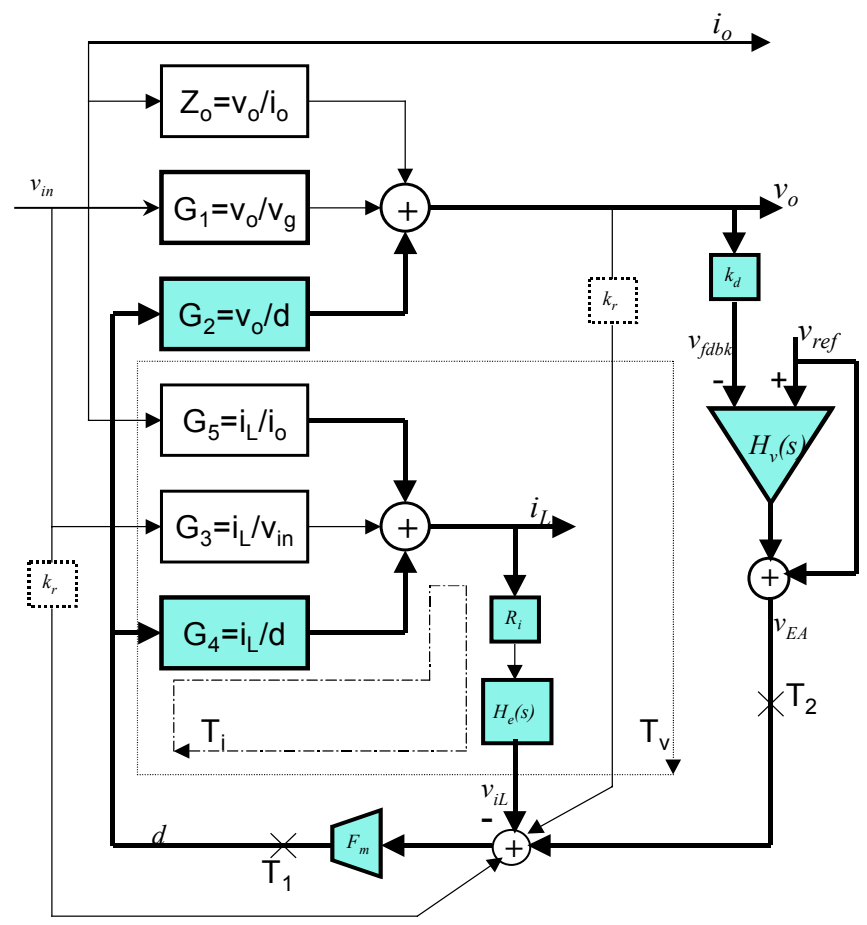

Figure 2.5-3 - Small Signal diagram of a DC/DC converter including the reference voltage terms 
Loop gain $T_{1}$ is referred to as the overall loop gain since it is measured inside both the current and voltage feedback loops, $T_{i}$ and $T_{v}$. The overall loop gain can be written as a combination of the current and voltage loops:

$$
T_{1}=T_{i}+T_{v}
$$

with

$$
T_{i}=F_{m} \cdot R_{i} \cdot H_{e}(s) \cdot G_{4}(s)
$$

and

$$
T_{v}=F_{m} \cdot k_{d} \cdot H_{v}(s) \cdot G_{2}(s)
$$

where, as explained above, the input voltage perturbation is considered zero and the output impedance, $Z_{o}$, is an internal network quantity from the loop gains view point. Loop gain $T_{2}$ is referred to as the outer loop gain and it is the most important quantity for the plant since it is similar to the " $G H$ " loop gain. It can be measured using conventional analog techniques, unlike $T_{1}$ that can only be measured using digital modulation techniques, [9], which is not of great importance for the study done here. In the absence of the reference term, $r=v_{\text {ref }}$, the outer loop gain can be written as

$$
T_{2}=\frac{T_{v}}{1+T_{i}}
$$

More details and explanatory plots are given in the analysis of the simulated results in Chapters 6.1 and 6.2 .

Considering now the reference term in Figure 2.5-3, we have

$v_{o}=G_{2} \cdot d=G_{2} \cdot F_{m} \cdot\left(v_{e a}-v_{i_{L}}\right)=F_{m} \cdot G_{2} \cdot\left[\left(1+H_{v}\right) \cdot r-H_{v} \cdot k_{d} \cdot v_{o}-G_{4} \cdot R_{i} \cdot H_{e} \cdot d\right]$

or, using (2.5-27) and (2.5-28),

$$
v_{o} \cdot\left(1+T_{v}+T_{i}\right)=F_{m} \cdot G_{2} \cdot\left(1+H_{v}\right) \cdot r .
$$

From equation (2.5-31) we obtain thus the Reference-to-Output transfer function

$$
G_{r}(s)=\frac{V_{o}(s)}{R(s)}=\frac{F_{m} \cdot G_{2}(s) \cdot\left(1+H_{v}(s)\right)}{1+T_{v}(s)+T_{i}(s)}=\frac{T_{v}(s)+F_{m} \cdot G_{2}(s)}{1+T_{v}(s)+T_{i}(s)} .
$$

If $r=0$, from (2.5-30), (2.5-27) and (2.5-28), we obtain the expression of $T_{2}$ in (2.5-29). 
It can be seen that the Reference-to-Output transfer function follows the structure of $T_{2}$ but includes a term due to the feed-through action of the OpAmp, from its non-inverting input, where $r$ is applied. Thus, a proportional type component is added in the CSC path, for the parallel system operation. As shown in [6], [9], except for individual blocks, the detailed closed form expressions are very lengthy and, depending on the PCMC gains modeling approach, require more or less simplifications in order to highlight the main quantities. However, block level Matlab and Simulink linearization can be employed in order to validate and use the above models, the results being shown in Chapter 6, both for small and large signal simulations. 


\section{Chapter 3}

\section{MODERN CONTROL SYSTEMS MODELING OF PARALLELED CONVERTER SYSTEMS}

Based on the disturbance rejection modeling approach introduced in Chapter 2, this chapter extends the same method to paralleled converter systems, with the objective of deriving a new, improved current sharing control method for master-slave type systems using fuzzy logic control.

\subsection{The Master - Slave Current Sharing Control as a Tracking Problem}

Consider again the large signal model (PCMC) for one ideal converter, with the output disturbance in an explicit form, equation (2.5-25)

$$
\begin{aligned}
& {\left[\begin{array}{c}
\dot{\mathbf{x}} \\
\dot{\mathbf{x}}_{\mathbf{f}}
\end{array}\right]=\left[\begin{array}{c|c}
\left(\mathbf{A}-F_{m} \cdot \mathbf{B} \cdot \mathbf{F}_{\mathbf{i x}}\right)-F_{m} \cdot \Delta \mathbf{A} \cdot \mathbf{x} \cdot \mathbf{F}_{\mathbf{i x}} & F_{m} \cdot(\mathbf{B}+\Delta \mathbf{A} \cdot \mathbf{x}) \cdot \mathbf{C}_{\mathbf{f}} \\
{\left[\mathbf{0}-\mathbf{B}_{\mathbf{f}}\right]} & \mathbf{A}_{\mathbf{f}}
\end{array}\right] \cdot\left[\begin{array}{c}
\mathbf{x} \\
\mathbf{x}_{\mathbf{f}}
\end{array}\right]+\left[\begin{array}{c}
\mathbf{E}_{\mathbf{x}}^{\mathbf{i}_{0}} \\
\mathbf{0}
\end{array}\right] \cdot i_{o}+} \\
& +\left[\begin{array}{c}
F_{m} \cdot(\mathbf{B}+\Delta \mathbf{A} \cdot \mathbf{x}) \\
\mathbf{B}_{\mathbf{f}}
\end{array}\right] \cdot v_{r e f} \stackrel{n o t 1}{=} \mathbf{A}_{\mathrm{cl}} \cdot \mathbf{x}_{\mathrm{cl}}+\mathbf{E}_{\mathrm{cl}} \cdot w+\mathbf{B}_{\mathrm{cl}} \cdot r \stackrel{n o t 2}{=} f_{c l}\left(\mathbf{x}_{\mathrm{cl}}\right)+p_{c l}(\mathbf{x}) \cdot w+g_{c l}\left(\mathbf{x}_{\mathrm{cl}}\right) \cdot r
\end{aligned}
$$

Consider now a paralleled system comprised of two converters, of which converter \#1 is master and converter \#2 is slave. This covers all practical cases since even in a "maximum current master" type of control, [35], where the master's role rotates, there is only one master converter at a time. Denoting with "sub-1" the quantities related to that converter, we have

$$
\begin{aligned}
& \dot{\mathbf{x}}_{1}=f_{1}\left(\mathbf{x}_{1}\right)+p_{1}(x) \cdot w_{1} \\
& y_{1}=\mathbf{x}_{1}(2)=C \cdot \mathbf{x}_{1} \\
& \dot{\mathbf{x}}_{2}=f_{2}\left(\mathbf{x}_{2}\right)+p_{2}(x) \cdot w_{2}+g_{2}\left(\mathbf{x}_{2}\right) \cdot r_{2} . \\
& y_{2}=\mathbf{x}_{2}(2)=C \cdot \mathbf{x}_{2}
\end{aligned}
$$


The master's model does not have the term in the reference input since it does not receive any current sharing information. Electrically, the two converter's connection looks like in Figure $3.1-1$.

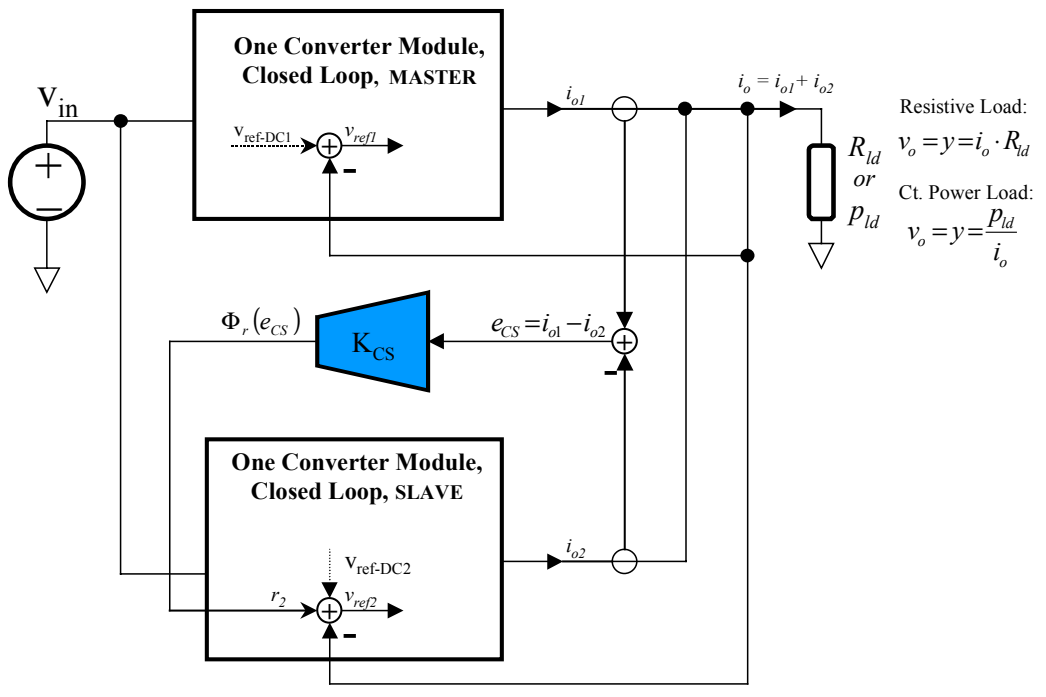

Figure 3.1-1 - Electrical Block Diagram-Schematic for two M-S control paralleled converters

Considering the unterminated model and writing the expression of the load current for Resistive Load (RL) and respectively Constant Power Load (CPL) for $n$ paralleled plants, we obtain the expression for each total, parallel system's output as

$$
\begin{aligned}
& v_{o}=R_{\text {load }} \cdot i_{\text {oLoad }}=\underbrace{R_{\text {load }} \cdot \sum_{i=1}^{n} i_{o i}}_{\sum_{v_{o i}}^{n} v_{o i}}, \quad \text { hence } y=\sum_{i=1}^{n} y_{i}, \text { for RL } \\
& v_{o}=\frac{P_{\text {load }}}{i_{\text {oLoad }}}=\frac{P_{\text {load }}}{\sum_{i=1}^{n} i_{o i}}=\frac{1}{\left(\sum_{i=1}^{n} \frac{i_{o i}}{P_{\text {load }}}\right)_{=\sum_{i=1}^{n} \frac{1}{v_{o i}}}}, \text { hence } y=\frac{1}{\sum_{i=1}^{n} \frac{1}{y_{i}}}, \text { for CPL }
\end{aligned}
$$

Hence, from a control systems standpoint, the diagram in Figure 3.1-1 can be drawn as in Figure 3.1-2 for RL and as in Figure 3.1-3 for CPL. 


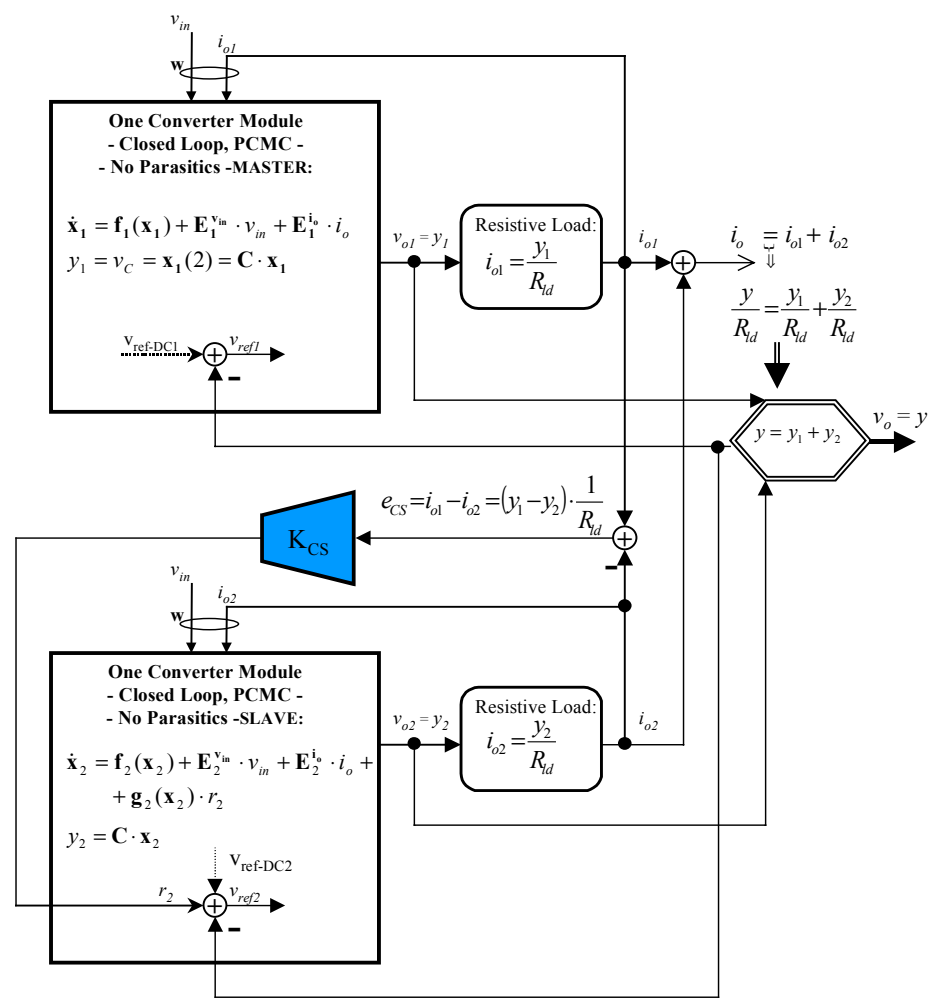

Figure 3.1-2- Control Systems Block Diagram-for two M-S control paralleled converter - Resistive Load

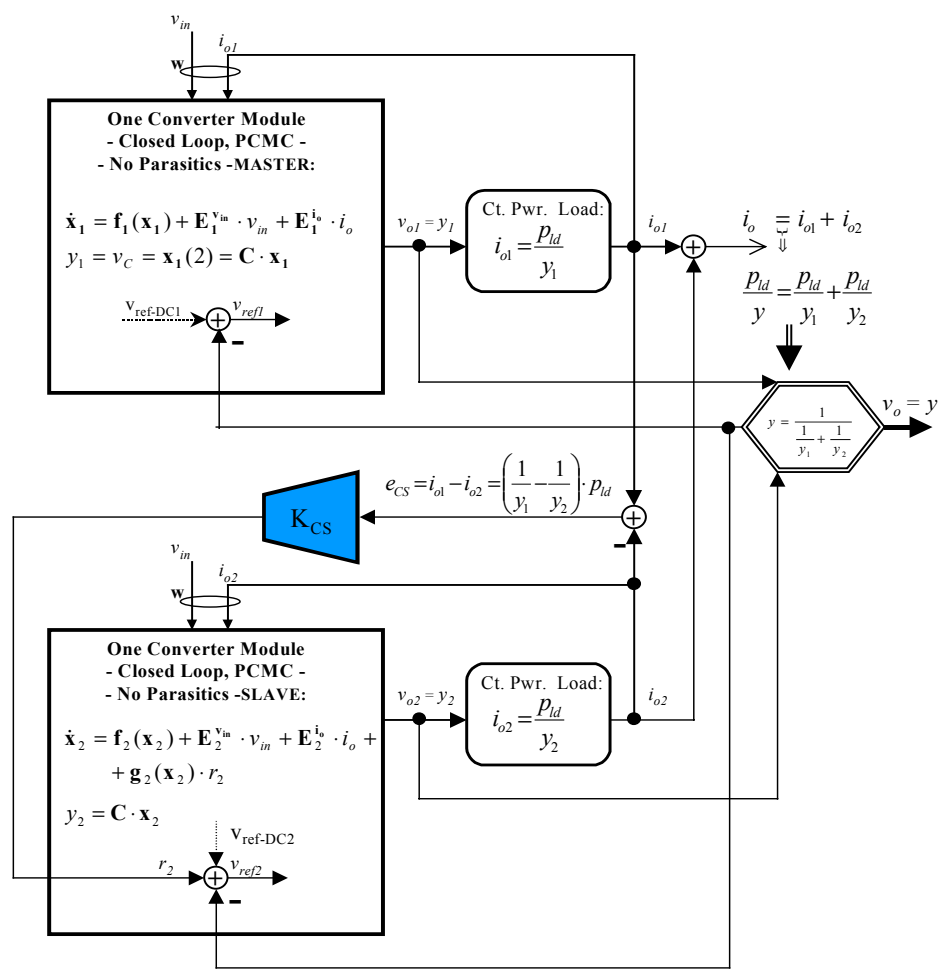

Figure 3.1-3- Control Systems Block Diagram-for two M-S control paralleled converter - Constant Power Load 
It can now be seen the importance of having both the electrical and the modern control systems interfaces, as they bring more light into the control problem. From examining the control scheme for both types of loads, one can see that for MSC, the error fed to the CSC is directly proportional to the state's second component error, ideally equal to the output voltage. Therefore, we are dealing with a tracking problem for a system of regulators, where the control problem is that of the slave tracking the master, once an output load or input voltage disturbance has occurred. The difference between the master's and slave's currents is altering the reference of the slave converter - normally on zero for the respective regulator - so it tracks the master converter output current. The slave is practically transformed in a controlled current source while the output voltage dynamics are regulated by the master converter, the only converter having its reference unaltered.

The CS error for the two load types disturbances can be written

$$
e_{c s}=\frac{1}{R_{l d}} \cdot e_{y}=\frac{1}{R_{l d}} \cdot \mathbf{C} \cdot \mathbf{e}_{\mathbf{x}}
$$

for RL and

$$
e_{c s}=p_{l d} \cdot \frac{e_{y}}{y_{1} \cdot y_{2}}=p_{l d} \cdot \frac{\mathbf{C} \cdot \mathbf{e}_{\mathbf{x}}}{\mathbf{C} \cdot \mathbf{x}_{\mathbf{1}} \cdot \mathbf{C} \cdot \mathbf{x}_{\mathbf{2}}}=p_{l d} \cdot \frac{\mathbf{C} \cdot \mathbf{e}_{\mathbf{x}}}{\mathbf{x}_{\mathbf{1}}(2) \cdot \mathbf{x}_{\mathbf{2}}(2)} .
$$

for CPL, with $\mathbf{e}_{\mathbf{x}}=\mathbf{x}_{1}-\mathbf{x}_{2}$. Using equation (2.5-17) we obtain for the states error equation in the case of the boost converter,

$\dot{\mathbf{e}}_{\mathbf{x}}=\left[\begin{array}{c}-V_{i n} \cdot M \cdot\left(F_{m 1} \cdot \frac{R_{i 1}}{L_{1}} \cdot x_{11}-F_{m 2} \cdot \frac{R_{i 2}}{L_{2}} \cdot x_{12}\right)-\left(F_{m 1} \cdot \frac{R_{i 1}}{L_{1}} \cdot x_{21} \cdot x_{11}-F_{m 2} \cdot \frac{R_{i 2}}{L_{2}} \cdot x_{22} \cdot x_{12}\right)-D^{\prime} \cdot\left(\frac{x_{21}}{L_{1}}-\frac{x_{22}}{L_{2}}\right)+ \\ +V_{i n} \cdot M \cdot\left(F_{m 1} \cdot \frac{\omega_{i 1}}{L_{1}} \cdot x_{f 21}-F_{m 2} \cdot \frac{\omega_{i 2}}{L_{2}} \cdot x_{f 22}\right)+\left(F_{m 1} \cdot \frac{\omega_{i 1}}{L_{1}} \cdot x_{21} \cdot x_{f 21}-F_{m 2} \cdot \frac{\omega_{i 2}}{L_{2}} \cdot x_{22} \cdot x_{f 22}\right) \\ D^{\prime} \cdot\left(\frac{x_{11}}{C_{1}}-\frac{x_{12}}{C_{2}}\right)+\frac{V_{i n} \cdot M}{D^{\prime} \cdot R_{l d}} \cdot\left(F_{m 1} \cdot \frac{R_{i 1}}{C_{1}} \cdot x_{11}-F_{m 2} \cdot \frac{R_{i 2}}{C_{2}} \cdot x_{12}\right)+\left(F_{m 1} \cdot \frac{R_{i 1}}{C_{1}} \cdot x_{11}^{2}-F_{m 2} \cdot \frac{R_{i 2}}{C_{2}} \cdot x_{12}^{2}\right)-\frac{1}{R_{l d}} \cdot\left(\frac{x_{21}}{C_{1}}-\frac{x_{22}}{C_{2}}\right) \\ -\frac{V_{i n} \cdot M}{D^{\prime} \cdot R_{l d}} \cdot\left(F_{m 1} \cdot \frac{\omega_{i 1}}{C_{1}} \cdot x_{f 21}-F_{m 2} \cdot \frac{\omega_{i 2}}{C_{2}} \cdot x_{f 22}\right)-\left(F_{m 1} \cdot \frac{\omega_{i 1}}{C_{1}} \cdot x_{11} \cdot x_{f 21}-F_{m 2} \cdot \frac{\omega_{i 2}}{C_{2}} \cdot x_{11} \cdot x_{f 21}\right) \\ -\left(x_{21}-x_{22}\right)-\left(\omega_{p 11} \cdot x_{f 11}-\omega_{p 12} \cdot x_{f 12}\right) \\ -\left(x_{21}-x_{22}\right)+\left(\omega_{z 11}-\omega_{p 11}\right) \cdot x_{f 11}-\left(\omega_{z 12}-\omega_{p 12}\right) \cdot x_{f 12}+\left(x_{f 21}-x_{f 22}\right)\end{array}\right]-\left[\begin{array}{c}F_{m 2} \cdot \frac{1}{L_{2}} \cdot\left(V_{i n} \cdot M+x_{22}\right) \\ -F_{m 2} \cdot \frac{1}{C_{2}} \cdot\left(\frac{V_{i n} \cdot M}{D^{\prime} \cdot R_{l d}}+x_{12}\right) \\ 1 \\ 1\end{array}\right] \cdot r_{2}$

where the first index for states denotes the state number and the second index denotes the converter number. From equation (3.1-7) we can see that: 
a) The FLC has to be achieved through $r_{2}$ and to be stable.

b) The paralleled system state - and hence current sharing - error has a nonlinear, complicated expression, not directly amenable for obtaining a closed form control law, which fully justifies the fuzzy logic approach.

Therefore, the case of two ideally identical converters, which share the current, much more even, [27], [28], will be first considered. Using the closed loop, loaded, converter model, (2.5-15), the error equation can be written

$$
\begin{aligned}
& \dot{\mathbf{e}}=\left[\begin{array}{c}
\dot{\mathbf{x}}_{1}-\dot{\mathbf{x}}_{2} \\
\dot{\mathbf{x}}_{\mathbf{f} 1}-\dot{\mathbf{x}}_{\mathbf{f} 2}
\end{array}\right]=\left[\begin{array}{cc}
\mathbf{A}-F_{m} \cdot \mathbf{B} \cdot \mathbf{C}_{\mathbf{i}} \cdot\left(\mathbf{I}+\frac{D \cdot T_{s}}{2} \cdot \mathbf{A}_{\mathbf{1}}\right) & F_{m} \cdot \mathbf{B} \cdot \mathbf{C}_{\mathbf{f}} \\
{\left[\mathbf{0}-\mathbf{B}_{\mathbf{f}}\right]} & \mathbf{A}_{\mathbf{f}}
\end{array}\right] \cdot\left[\begin{array}{c}
\mathbf{e}_{\mathbf{x}} \\
\mathbf{e}_{\mathbf{x}_{\mathbf{f}}}
\end{array}\right]-\left[\begin{array}{c}
F_{m} \cdot\left(\mathbf{B}+\Delta \mathbf{A} \cdot \mathbf{x}_{\mathbf{2}}\right) \\
\mathbf{B}_{\mathbf{f}}
\end{array}\right] \cdot r+ \\
& +\left[\begin{array}{c}
-F_{m} \cdot \Delta \mathbf{A} \cdot \mathbf{x}_{\mathbf{1}} \cdot \mathbf{C}_{\mathbf{i}} \cdot\left(\mathbf{I}+\frac{D \cdot T_{s}}{2} \cdot \mathbf{A}_{\mathbf{1}}\right) \cdot \mathbf{x}_{\mathbf{1}}+F_{m} \cdot \Delta \mathbf{A} \cdot \mathbf{x}_{\mathbf{2}} \cdot \mathbf{C}_{\mathbf{i}} \cdot\left(\mathbf{I}+\frac{D \cdot T_{s}}{2} \cdot \mathbf{A}_{\mathbf{1}}\right) \cdot \mathbf{x}_{\mathbf{2}}+ \\
+F_{m} \cdot \Delta \mathbf{A} \cdot \mathbf{x}_{\mathbf{1}} \cdot \mathbf{C}_{\mathbf{f}} \cdot \mathbf{x}_{\mathbf{f} 1}-F_{m} \cdot \Delta \mathbf{A} \cdot \mathbf{x}_{\mathbf{2}} \cdot \mathbf{C}_{\mathbf{f}} \cdot \mathbf{x}_{\mathbf{f} 2} \\
\mathbf{0}
\end{array}\right]
\end{aligned}
$$

which can be further simplified by collecting the terms,

$$
\begin{aligned}
& \dot{\mathbf{e}}=\left[\begin{array}{l}
\dot{\mathbf{e}}_{\mathbf{x}} \\
\dot{\mathbf{e}}_{\mathbf{x}_{\mathbf{f}}}
\end{array}\right]=\left[\begin{array}{cc}
\mathbf{A}-F_{m} \cdot \mathbf{B} \cdot \mathbf{C}_{\mathbf{i}} \cdot\left(\mathbf{I}+\frac{D \cdot T_{s}}{2} \cdot \mathbf{A}_{\mathbf{1}}\right) & F_{m} \cdot \mathbf{B} \cdot \mathbf{C}_{\mathbf{f}} \\
{\left[\mathbf{0}-\mathbf{B}_{\mathbf{f}}\right.}
\end{array}\right] \cdot\left[\begin{array}{c}
\mathbf{e}_{\mathbf{x}} \\
\mathbf{e}_{\mathbf{x}_{\mathbf{f}}}
\end{array}\right]-\left[\begin{array}{c}
F_{m} \cdot\left(\mathbf{B}+\Delta \mathbf{A} \cdot \mathbf{x}_{\mathbf{2}}\right) \\
\mathbf{B}_{\mathbf{f}}
\end{array}\right] \cdot r+ \\
& +\left[\begin{array}{c}
F_{m} \cdot \Delta \mathbf{A} \cdot\left(\mathbf{x}_{\mathbf{2}} \cdot \mathbf{C}_{\mathbf{i}} \cdot\left(\mathbf{I}+\frac{D \cdot T_{s}}{2} \cdot \mathbf{A}_{\mathbf{1}}\right) \cdot \mathbf{x}_{\mathbf{2}}-\mathbf{x}_{\mathbf{1}} \cdot \mathbf{C}_{\mathbf{i}} \cdot\left(\mathbf{I}+\frac{D \cdot T_{s}}{2} \cdot \mathbf{A}_{\mathbf{1}}\right) \cdot \mathbf{x}_{\mathbf{1}}+\right) \\
+\mathbf{x}_{\mathbf{1}} \cdot \mathbf{C}_{\mathbf{f}} \cdot \mathbf{x}_{\mathbf{f} 1}-\mathbf{x}_{\mathbf{2}} \cdot \mathbf{C}_{\mathbf{f}} \cdot \mathbf{x}_{\mathbf{f} 2} \\
\mathbf{0}
\end{array}\right]
\end{aligned}
$$

Equations (3.1-9) and (3.1-7) highlight now better the paralleled system's structure in terms of the current sharing error. The analysis and design of the CSC can be done employing equation (3.1-9), which offers a clearer analytical form, while the validity and fine tuning of the design can then be done using the complete model, (3.1-7), which considers the parameters variation, 
among the converters. In addition, this form will be updated in simulations to include all the parasitics, in an unterminated model formalism. 


\section{Chapter 4}

\section{LYAPUNOV STABILITY ANALYSIS FOR DC/DC REGULATORS}

This chapter discusses the stability issues and properties that DC/DC regulators have from a Lyapunov energy function perspective. The main reason for the approach is that, even though sometimes too stringent, when available, Lyapunov functions offer stable trajectories for any control method desired. This is particularly important for fuzzy logic control methods of complex systems, i.e., MSC of paralleled converters current sharing.

\subsection{Open Loop Stability under Resistive Load}

Let the switching converter be composed of ideal DC sources, ideal switches, incrementally passive resistors, [13] and linear passive reactive elements. Consider the currents and voltages in each network branch with respect to the nominal OP trajectory solution and their increments (perturbations), $d i, d v$. Tellegen's theorem states that they are orthogonal, both as full quantities and as increments. We obtain thus,

$$
\sum_{D C \text { Sources }} d i \cdot d v+\sum_{\text {Switches }} d i \cdot d v+\sum_{\text {"Positive"Re sistors }} d i \cdot d v+\sum_{\text {Inductors }} d i \cdot d v+\sum_{\text {Capacitors }} d i \cdot d v=0
$$

The summations in the terms involving DC sources and Switches are zero as the increment in either voltage or current of each term is necessarily zero. The summation for "Positive Resistors" is always non-negative as each term is individually zero or positive, as a result of incremental passivity of resistors. This summation can be thought of as the dissipated power in the increment with respect to the given nominal trajectory. The terms concerning the reactive, passive inductors and capacitors, represent the time rate of change, $\dot{\mathbf{V}}(d \mathbf{x})$, of the stored energy in the increment, $\mathbf{V}(d \mathbf{x})$,

$$
\mathbf{V}(d \mathbf{x})=\sum_{\text {Ind. }} \frac{1}{2} \cdot d i \cdot d v+\sum_{\text {Cap. }} \frac{1}{2} \cdot d i \cdot d v
$$


which is a positive definite quadratic function of the perturbations of the state variables. Because (4.1-1) holds identically for any of the possible circuit topologies, the energy in the increment is a Lyapunov function for the given nominal trajectory From identifying the energy rate of change, $\dot{\mathbf{V}}(d \mathbf{x})$ and equations (4.1-1) and (4.1-2) we have that

$$
\dot{\mathbf{V}}(d \mathbf{x})=-\sum_{\text {"Positive" Re sistors }} d i \cdot d v \leq 0
$$

and hence the nominal trajectory is Lyapunov stable, [13]. Moreover, asymptotic stability can be concluded as well as at least some parasitic loss is always associated with each inductor and capacitor and hence the inequality in (4.1-3) becomes strict. Because the nominal trajectory above can be taken as any solution trajectory, we have that any two solutions trajectories do not diverge. It is also depicted in [13] that the case when the switches are operated with a periodic switching pattern of finite frequency can be extended to the case where the switching frequency becomes infinite. This is the case naturally analyzed with the state-space averaged model of the converter, since it would idealize the assumptions of having the time constants of the circuit much larger than the switching frequency, which are the modeling premises discussed in chapter 2. In this way, an open-loop stability result is obtained for state-space averaged models. The main difference between the case of finite switching frequency is that only the stability of an equilibrium point for an averaged model needs to be considered rather than a limit cycle. Therefore, we are able now to include nonlinear reactive and resistive elements that are of interest for the practical cases in power electronics circuits.

\subsection{Open loop stability under Constant Power Load}

As detailed in Chapter 2, a constant power load introduces a non-passive, negative equivalent (incremental) resistance behavior and (4.1-1) will now have an additional, CPL - $p_{l d}$, term for "Negative Resistors"

$$
\sum_{\text {Negative" Re sistors }}-d i \cdot d v \equiv \sum_{C P L}-d i \cdot d v=\sum_{C P L}-d i \cdot \frac{p_{l d}}{d i}=\sum_{C P L} p_{l d}>0
$$


which will modify (4.1-3) as

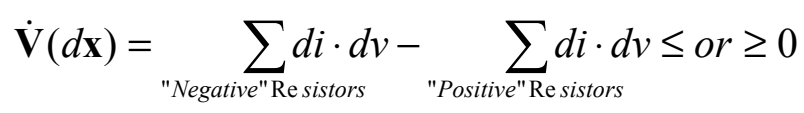

depending on the sign of the difference between the equivalent negative resistances value and the positive resistances value.

\subsection{Use of a Lyapunov approach for individual module feedback design}

Recognizing that the vector $d \mathbf{x}$ and its components $d i, d v$ above are actually the dynamic perturbations from nominal employed throughout this study, i.e., $\mathbf{x}$ and respectively $i_{L}$ and $v_{C}$, we can now use our previous models in the subsequent Laypunov analysis. Consider again the system's model for the states, as given in equations (2.5-1), (2.5-2) and including the input voltage perturbation, for completeness,

$$
\begin{aligned}
\dot{\mathbf{x}} & =\left[\begin{array}{cc}
0 & -\frac{(1-D)}{L} \\
\frac{(1-D)}{C} & -\frac{1}{R_{l d} \cdot C}
\end{array}\right] \cdot \mathbf{x}+V_{i n} \cdot M \cdot\left[\begin{array}{cc}
\frac{1}{L} \\
-\cdot \frac{1}{(1-D)} \cdot \frac{1}{R_{l d} \cdot C}
\end{array}\right] \cdot u+\left[\begin{array}{c}
\frac{1}{L} \\
0
\end{array}\right] \cdot v_{i n}+\left[\begin{array}{cc}
0 & \frac{1}{L} \\
-\frac{1}{C} & 0
\end{array}\right] \cdot \mathbf{x} \cdot u= \\
& =\mathbf{A} \cdot \mathbf{x}+\mathbf{B} \cdot u+\mathbf{E} \cdot v_{i n}+\Delta \mathbf{A} \cdot \mathbf{x} \cdot u
\end{aligned}
$$

Without loss of generality, we will consider that the load resistance includes the sign, i.e., positive for resistive load and negative for constant power load. As it was shown in paragraph 4.1, equation (4.1-2) the stored energy in the increment, in the reactive elements - inductors and capacitors - giving the state of the system, is a Lyapunov function for the open loop, passive plant. Therefore, we can write its expression in the quadratic form, [Appendix B],

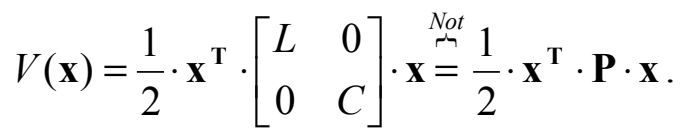

and its derivative becomes

$$
\dot{V}(\mathbf{x})=\frac{1}{2} \cdot \mathbf{x}^{\mathbf{T}} \cdot\left(\mathbf{P} \cdot \mathbf{A}+\mathbf{A}^{\mathbf{T}} \cdot \mathbf{P}\right) \cdot \mathbf{x}+\mathbf{x}^{\mathbf{T}} \cdot \mathbf{P} \cdot \mathbf{B} \cdot u+\mathbf{x}^{\mathbf{T}} \cdot \mathbf{P} \cdot \mathbf{E} \cdot v_{i n}+\frac{1}{2} \cdot \mathbf{x}^{\mathbf{T}} \cdot\left(\mathbf{P} \cdot \Delta \mathbf{A}+\Delta \mathbf{A}^{\mathbf{T}} \cdot \mathbf{P}\right) \cdot \mathbf{x} \cdot u
$$

or, using (4.3-1) and (4.3-2), 


$$
\dot{V}(\mathbf{x})=-\frac{1}{R_{l d}} \cdot x_{2}^{2}+V_{i n} \cdot M \cdot\left(x_{1}-\frac{x_{2}}{(1-D)} \cdot \frac{1}{R_{l d}}\right) \cdot u+x_{1} \cdot v_{i n}+0
$$

Equation (4.3-4) reveals several important facts about the open loop system and its energy function. First, it shows that investigating the open loop bilinear, loss-less, system Lyapunov stability by employing this energy function is the same as investigating the linear system since the last term on the right side of equation is zero due to the $\Delta \mathbf{A}$ matrix in (4.3-1) being skew-symmetric.

Secondly, it verifies, as in [13], that the worst case for stability under a passive resistor load is for infinite load, i.e., the first term on the right side equal to zero; in that case, the $\mathbf{A}$ matrix itself is also skew symmetric. It can be seen however that for a CPL, equivalent to an active (negative) resistor load, the first term actually hinders substantially this energy function's derivative from being negative definite. As mentioned in Chapter 2, there is no universal approach, to accommodate for all type of variations for both resistive and constant power loads, unless the controllers and/or the systems become very complicated. This does not mean that the real system is always unstable, in practice it is actually stable most times, provided that the load variations are small and the converter has good phase and gain margins. The parasitics also play an important role, helping the stability through the damping that they introduce, here a worst case no damping system is considered. In addition, practical CPLs always have an equivalent, high value, resistive load in parallel wit them, especially at low voltages. The subsequent study will address the resistive load case as a starting point for developing the new fuzzy control algorithm, which is the main focus of this study. Constant power loads require additional developments, but once the design approach for the fuzzy logic compensator is introduced in the simpler case of a resistive load they can be treated similarly and can be the subject of future research.

Third, as mentioned previously, since this study is concerned with output load disturbance reduction, the perturbation in $v_{\text {in }}$ will be considered zero. Solving the problem of output (disturbance) current sharing is of higher importance and more difficult due to its way of coming into the plant's structure. The input voltage comes in linearly and is a completely exogenous quantity, unlike the load disturbance. Hence its study is more straight forward and can be considered for future research. 
Fourth, the term in the control, $u$, links the open loop plant stability to the closed loop one and thus to the paralleled system current sharing stability, as it will be shown in the next paragraph, detailing the closed loop model.

All the above conclusions hold for all six basic converters, as it can be easily verified in the existing literature and therefore they prove the generality of the approach.

Considering, again, the term in the control, $u$, in equation (4.3-4), one of the first approaches to a Lyapunov stable control was for a passive resistive load, where the control is determined so it guarantees that the derivative of the energy function in (4.3-4) is negative, [13]. That approach addressed only single converter modules, already well established in the industry in terms of control implementation and size, where a complex controller is not suitable when compared with the present OpAmp compensators, all with very good performance, small size and price. In the case of distributed systems however, due to their complexity and size allowance, modern digital controllers are fully justified and needed as the next step in DC/DC power conversion. It is therefore important to investigate the Lyapunov stability properties of the complete, closed loop, DC/DC regulator.

Since, as mentioned before, the worst case is for the loss-less network, the design oriented analysis will continue for the models with no parasitics and the simulated results will be done using the real system.

\subsection{Closed Loop Lyapunov Stability Analysis}

Regarding the paralleled system control, this DC/DC regulator is used as having the reference voltage input as its new control input. Returning to the case of interest in this study and recalling equation (2.5-15) we have the closed loop model

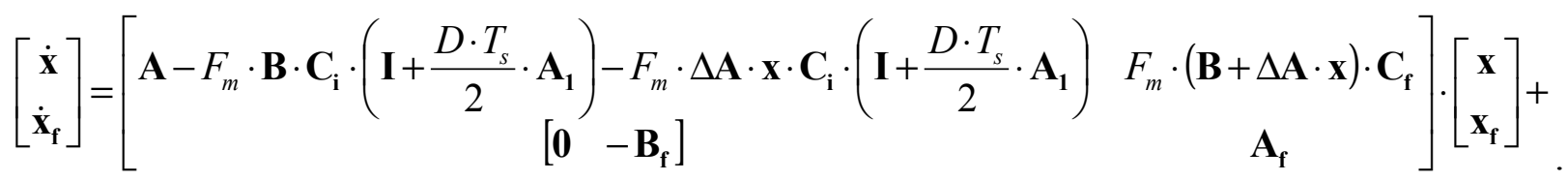

$$
\begin{aligned}
& +\left[\begin{array}{c}
F_{m} \cdot(\mathbf{B}+\Delta \mathbf{A} \cdot \mathbf{x}) \\
\mathbf{B}_{\mathbf{f}}
\end{array}\right] \cdot v_{r e f}=\mathbf{A}_{\mathbf{c l}} \cdot \mathbf{x}_{\mathrm{cl}}+\mathbf{B}_{\mathrm{cl}} \cdot r
\end{aligned}
$$


Using the same type of Lyapunov function as in (4.3-2) above, but accounting also for the compensator states, Fig. 2.5-1 and equations (2.5-10) and (2.5-11), we take

$$
\mathbf{P}_{\mathbf{c l}}=\left[\begin{array}{cccc}
L & 0 & 0 & 0 \\
0 & C & 0 & 0 \\
0 & 0 & C_{1} & 0 \\
0 & 0 & 0 & C_{3}
\end{array}\right] \stackrel{\substack{n o t \\
\sim}}{=}\left[\begin{array}{cc}
\mathbf{P}_{\mathbf{x}} & \mathbf{0} \\
\mathbf{0} & \mathbf{P}_{\mathbf{x}_{\mathbf{f}}}
\end{array}\right] .
$$

The derivative of the energy function can be written now

$$
\dot{V}_{c l}(\mathbf{x})=\frac{1}{2} \cdot \mathbf{x}_{\mathrm{cl}}^{\mathrm{T}} \cdot\left(\mathbf{P}_{\mathrm{cl}} \cdot \mathbf{A}_{\mathrm{cl}}+\mathbf{A}_{\mathrm{cl}}^{\mathrm{T}} \cdot \mathbf{P}_{\mathrm{cl}}\right) \cdot \mathbf{x}_{\mathrm{cl}}+\mathbf{x}_{\mathrm{cl}}^{\mathrm{T}} \cdot \mathbf{P}_{\mathrm{cl}} \cdot \mathbf{B}_{\mathrm{cl}} \cdot r
$$

or, after expanding the terms,

$$
\begin{aligned}
& \dot{V}_{c l}(\mathbf{x})=\frac{1}{2} \cdot\left[\begin{array}{ll}
\mathbf{x}^{\mathbf{T}} & \mathbf{x}_{\mathbf{f}}^{\mathbf{T}}
\end{array}\right] \cdot\left[\begin{array}{cc}
\mathbf{a}_{11} & F_{m} \cdot\left(\mathbf{P}_{\mathbf{x}} \cdot(\mathbf{B}+\Delta \mathbf{A} \cdot \mathbf{x}) \cdot \mathbf{C}_{\mathbf{f}}+\mathbf{C}_{\mathbf{f}}^{\mathbf{T}} \cdot(\mathbf{B}+\Delta \mathbf{A} \cdot \mathbf{x})^{\mathbf{T}} \cdot \mathbf{P}_{\mathbf{x}}\right) \\
\mathbf{P}_{\mathbf{x}_{\mathbf{f}}} \cdot\left[\begin{array}{cc}
\mathbf{0} & \left.-\mathbf{B}_{\mathbf{f}}\right]
\end{array}\right]+\left[\begin{array}{ll}
\mathbf{0} & -\mathbf{B}_{\mathbf{f}}
\end{array}\right]^{\mathbf{T}} \cdot \mathbf{P}_{\mathbf{x}_{\mathbf{f}}} & \mathbf{P}_{\mathbf{x}_{\mathbf{f}}} \cdot \mathbf{A}_{\mathbf{f}}+\mathbf{A}_{\mathbf{f}}^{\mathbf{T}} \cdot \mathbf{P}_{\mathbf{x}_{\mathbf{f}}}
\end{array}\right] \cdot\left[\begin{array}{l}
\mathbf{x} \\
\mathbf{x}_{\mathbf{f}}
\end{array}\right]+ \\
& +\left[\begin{array}{ll}
\mathbf{x}^{\mathbf{T}} & \mathbf{x}_{\mathbf{f}}^{\mathbf{T}}
\end{array}\right] \cdot\left[\begin{array}{c}
\mathbf{P}_{\mathbf{x}} \cdot F_{m} \cdot(\mathbf{B}+\Delta \mathbf{A} \cdot \mathbf{x}) \\
\mathbf{P}_{\mathbf{x}_{\mathbf{f}}} \cdot \mathbf{B}_{\mathbf{f}}
\end{array}\right] \cdot r
\end{aligned}
$$

with $\mathbf{a}_{11}$ given by

$$
\begin{aligned}
\mathbf{a}_{11}= & \left(\mathbf{P}_{\mathbf{x}} \cdot \mathbf{A}+\mathbf{A}^{T} \cdot \mathbf{P}_{\mathbf{x}}\right)-F_{m} \cdot\left(\mathbf{P}_{\mathbf{x}} \cdot \mathbf{B} \cdot \mathbf{C}_{\mathbf{i}}+\mathbf{C}_{\mathbf{i}}^{\mathbf{T}} \cdot \mathbf{B}^{\mathbf{T}} \cdot \mathbf{P}_{\mathbf{x}}\right)-F_{m} \cdot \frac{D \cdot T_{s}}{2} \cdot\left(\mathbf{P}_{\mathbf{x}} \cdot \mathbf{B} \cdot \mathbf{C}_{\mathbf{i}} \cdot \mathbf{A}_{\mathbf{1}}+\mathbf{A}_{\mathbf{1}}^{\mathbf{T}} \cdot \mathbf{C}_{\mathbf{i}}^{\mathbf{T}} \cdot \mathbf{B}^{\mathbf{T}} \cdot \mathbf{P}_{\mathbf{x}}\right)- \\
& -F_{m} \cdot\left(\mathbf{P}_{\mathbf{x}} \cdot \Delta \mathbf{A} \cdot \mathbf{x} \cdot \mathbf{C}_{\mathbf{i}}+\mathbf{C}_{\mathbf{i}}^{\mathbf{T}} \cdot \mathbf{x}^{\mathbf{T}} \cdot \Delta \mathbf{A}^{\mathbf{T}} \cdot \mathbf{P}_{\mathbf{x}}\right)-F_{m} \cdot \frac{D \cdot T_{s}}{2} \cdot\left(\mathbf{P}_{\mathbf{x}} \cdot \Delta \mathbf{A} \cdot \mathbf{x} \cdot \mathbf{C}_{\mathbf{i}} \cdot \mathbf{A}_{\mathbf{1}}+\mathbf{A}_{\mathbf{1}}^{\mathbf{T}} \cdot \mathbf{C}_{\mathbf{i}}^{\mathbf{T}} \cdot \mathbf{x}^{\mathbf{T}} \cdot \Delta \mathbf{A}^{\mathbf{T}} \cdot \mathbf{P}_{\mathbf{x}}\right)
\end{aligned}
$$

Except the first two, all the terms in (4.4-5) are zero for the elementary, loss-less topologies, i.e., buck, boost, buck-boost since either $\Delta \mathbf{A}$ is zero (buck) and/or $\mathbf{A}_{\mathbf{1}}$ is skew symmetric "in all terms" except the last one of the main diagonal, which is proportional with the load resistance. $\Delta \mathbf{A}$ is also skew symmetric for all basic DC-DC topologies, [39], [40]. The derivative of the energy function becomes thus 


$$
\begin{aligned}
& \dot{V}_{c l}(\mathbf{x})=\frac{1}{2} \cdot \mathbf{x}^{\mathrm{T}} \cdot\left[\left(\mathbf{P}_{\mathbf{x}} \cdot \mathbf{A}+\mathbf{A}^{T} \cdot \mathbf{P}_{\mathbf{x}}\right)_{\mathrm{I}}-F_{m} \cdot\left(\mathbf{P}_{\mathbf{x}} \cdot \mathbf{B} \cdot \mathbf{C}_{\mathbf{i}}+\mathbf{C}_{\mathbf{i}}^{\mathrm{T}} \cdot \mathbf{B}^{\mathbf{T}} \cdot \mathbf{P}_{\mathbf{x}}\right)_{I I}\right] \cdot \mathbf{x}+\frac{1}{2} \cdot \mathbf{x}_{\mathbf{f}}^{\mathbf{T}} \cdot\left(\mathbf{P}_{\mathbf{x}_{\mathrm{f}}} \cdot \mathbf{A}_{\mathbf{f}}+\mathbf{A}_{\mathbf{f}}^{\mathbf{T}} \cdot \mathbf{P}_{\mathbf{x}_{\mathrm{f}}}\right)_{\mathrm{III}} \cdot \mathbf{x}_{\mathbf{f}}+ \\
& +\frac{1}{2} \cdot F_{m} \cdot \mathbf{x}^{\mathrm{T}} \cdot\left[\left(\mathbf{P}_{\mathbf{x}} \cdot \mathbf{B} \cdot \mathbf{C}_{\mathbf{f}}+\mathbf{C}_{\mathbf{f}}{ }^{\mathrm{T}} \cdot \mathbf{B}^{\mathrm{T}} \cdot \mathbf{P}_{\mathbf{x}}\right)_{\mathrm{IV}}+\left(\mathbf{P}_{\mathbf{x}} \cdot \Delta \mathbf{A} \cdot \mathbf{x} \cdot \mathbf{C}_{\mathbf{f}}+\mathbf{C}_{\mathbf{f}}{ }^{\mathrm{T}} \cdot \mathbf{x}^{\mathrm{T}} \cdot \Delta \mathbf{A}^{\mathrm{T}} \cdot \mathbf{P}_{\mathbf{x}}\right)_{\mathrm{V}}\right] \cdot \mathbf{x}_{\mathbf{f}}+ \\
& +\frac{1}{2} \cdot \mathbf{x}_{\mathbf{f}}^{\mathrm{T}} \cdot\left(\mathbf{P}_{\mathbf{x}_{\mathrm{f}}} \cdot\left[\begin{array}{ll}
\mathbf{0} & -\mathbf{B}_{\mathbf{f}}
\end{array}\right]+\left[\begin{array}{ll}
\mathbf{0} & -\mathbf{B}_{\mathrm{f}}
\end{array}\right]^{\mathrm{T}} \cdot \mathbf{P}_{\mathbf{x}_{\mathrm{f}}}\right)_{\mathrm{VII}} \cdot \mathbf{x}+\left[\mathbf{x}^{\mathrm{T}} \cdot \mathbf{P}_{\mathbf{x}} \cdot F_{m} \cdot(\mathbf{B}+\Delta \mathbf{A} \cdot \mathbf{x})_{\mathrm{VII}}+\left(\mathbf{x}_{\mathbf{f}}^{\mathrm{T}} \cdot \mathbf{P}_{\mathbf{x}_{\mathrm{f}}} \cdot \mathbf{B}_{\mathbf{f}}\right)_{\mathrm{VIII}}\right] \cdot r
\end{aligned}
$$

where the subscripts in roman numerals were added for subsequent cross references. Detailing the terms for the boost converter gives (almost identical for a buck-boost and similar for the buck converter)

$$
\begin{aligned}
\dot{V}_{c l}(\mathbf{x})= & -\left.\frac{1}{R_{l d}} \cdot x_{2}^{2}\right|_{\mathrm{I}}-F_{m} \cdot V_{i n} \cdot M \cdot R_{i} \cdot\left(x_{1}^{2}-\frac{1}{D^{\prime} \cdot R_{l d}} \cdot x_{1} \cdot x_{2}\right)_{\mathrm{II}}+\left[-\left(1+\frac{C_{1}}{C_{3}}\right) \cdot \frac{1}{R_{2}} \cdot x_{f 1}^{2}-\frac{1}{2} \cdot \frac{1}{R_{2}} \cdot x_{f 1}^{2}+\frac{1}{2} \cdot \frac{1}{R_{2}} \cdot x_{f 1} \cdot x_{f 2}\right]_{\mathrm{III}} \\
& +\frac{1}{2} \cdot F_{m} \cdot V_{i n} \cdot M \cdot \omega_{i} \cdot\left(x_{1} \cdot x_{f 2}+x_{2} \cdot x_{f 1}-\frac{2}{D^{\prime} \cdot R_{l d}} \cdot x_{2} \cdot x_{f 2}\right)_{\mathrm{IV}}+\frac{1}{2} \cdot \omega_{i} \cdot F_{m} \cdot x_{2} \cdot\left(x_{2} \cdot x_{f 1}-x_{1} \cdot x_{f 2}\right)_{\mathrm{V}}- \\
& -\left[\frac{1}{2} \cdot C_{1} \cdot\left(x_{2} \cdot x_{f 1}+x_{1} \cdot x_{f 2}\right)+C_{3} \cdot x_{2} \cdot x_{f 2}\right]_{\mathrm{VI}}+\left[F_{m} \cdot V_{i n} \cdot M \cdot\left(x_{1}-\frac{x_{2}}{D^{\prime}} \cdot \frac{1}{R_{l d}}\right)_{\mathrm{VII}}+\left(C_{1} \cdot x_{f 1}+C_{3} \cdot x_{f 2}\right)_{\mathrm{VIII}}\right] \cdot r
\end{aligned}
$$

where one can now reference which term in (4.4-6) corresponds to each of the terms in (4.4-7), through the roman numerals indices. For the individual regulator, $r=0$, and the last terms disappears; it was kept in because it is used as the control input for the slave converter in the master-slave parallel control scheme, which is analyzed subsequently. If, in addition, we neglect the small terms - also negative - containing the two OpAmp compensator capacitors and resistor we obtain

$$
\begin{aligned}
& \dot{V}_{c l}(\mathbf{x})=-\left.\frac{1}{R_{l d}} \cdot x_{2}^{2}\right|_{\mathrm{I}}-F_{m} \cdot V_{i n} \cdot M \cdot R_{i} \cdot\left(x_{1}^{2}-\frac{1}{D^{\prime} \cdot R_{l d}} \cdot x_{1} \cdot x_{2}\right)_{\mathrm{II}}+F_{m} \cdot V_{i n} \cdot M \cdot\left(x_{1}-\frac{x_{2}}{D^{\prime}} \cdot \frac{1}{R_{l d}}\right)_{\mathrm{VII}} \cdot r+ \\
& +F_{m} \cdot V_{i n} \cdot M \cdot \omega_{i} \cdot\left[\frac{1}{2} \cdot\left(x_{1} \cdot x_{f 2}+x_{2} \cdot x_{f 1}\right)-\frac{1}{D^{\prime} \cdot R_{l d}} \cdot x_{2} \cdot x_{f 2}\right]_{\mathrm{IV}}+\frac{1}{2} \cdot \omega_{i} \cdot F_{m} \cdot x_{2} \cdot\left(x_{2} \cdot x_{f 1}-x_{1} \cdot x_{f 2}\right)_{\mathrm{V}}
\end{aligned}
$$

Similarly with the open loop conclusions, relation (4.4-7) shows that the nature of the load resistance plays a vital role in determining the sign of the Lyapunov function derivative. It can 
actually be seen that the first term in the expression of $\dot{V}_{c l}(\mathbf{x})$ is the instantaneous output power with a minus sign. For the resistive load case, obviously, the output power is positive and hence the first term is negative. So is term "II", since $x_{1}$ and $x_{2}$, the inductor current and capacitor (output) voltage, vary in opposite directions from nominal, in what concerns the second factor in "II". The terms multiplied by the compensator capacitors are very small and most of them negative. Each term can be investigated and plotted in greater detail. But, for an individual module that is a passive network with all the poles in the LHP, the converter will be Lyapunov stable. For the paralleled system, however, even for resistive load, the two converters - each of them being still a constant power load - interact and a more detailed analysis of the energy function derivative terms is needed; this is the subject of the next section.

\subsection{Lyapunov Stability for two identical parallel converters}

Considering two ideal, identical, converters and using the state error equation (3.1-9) reproduced here for cross reference,

$$
\begin{aligned}
& \dot{\mathbf{e}}=\left[\begin{array}{c}
\dot{\mathbf{e}}_{\mathbf{x}} \\
\dot{\mathbf{e}}_{\mathbf{x}_{\mathbf{f}}}
\end{array}\right]=\left[\begin{array}{cc}
\mathbf{A}-F_{m} \cdot \mathbf{B} \cdot \mathbf{C}_{\mathbf{i}} \cdot\left(\mathbf{I}+\frac{D \cdot T_{s}}{2} \cdot \mathbf{A}_{\mathbf{1}}\right) & F_{m} \cdot \mathbf{B} \cdot \mathbf{C}_{\mathbf{f}} \\
{\left[\mathbf{0}-\mathbf{B}_{\mathbf{f}}\right]} & \mathbf{A}_{\mathbf{f}}
\end{array}\right] \cdot\left[\begin{array}{l}
\mathbf{e}_{\mathbf{x}} \\
\mathbf{e}_{\mathbf{x}_{\mathrm{f}}}
\end{array}\right]-\left[\begin{array}{c}
F_{m} \cdot\left(\mathbf{B}+\Delta \mathbf{A} \cdot \mathbf{x}_{\mathbf{2}}\right) \\
\mathbf{B}_{\mathbf{f}}
\end{array}\right] \cdot r+ \\
& +\left[\begin{array}{c}
F_{m} \cdot \Delta \mathbf{A} \cdot\left(\mathbf{x}_{\mathbf{2}} \cdot \mathbf{C}_{\mathbf{i}} \cdot\left(\mathbf{I}+\frac{D \cdot T_{s}}{2} \cdot \mathbf{A}_{\mathbf{1}}\right) \cdot \mathbf{x}_{\mathbf{2}}-\mathbf{x}_{\mathbf{1}} \cdot \mathbf{C}_{\mathbf{i}} \cdot\left(\mathbf{I}+\frac{D \cdot T_{s}}{2} \cdot \mathbf{A}_{\mathbf{1}}\right) \cdot \mathbf{x}_{\mathbf{1}}+\right) \\
+\left(\mathbf{x}_{\mathbf{1}} \cdot \mathbf{C}_{\mathbf{f}} \cdot \mathbf{x}_{\mathbf{f} 1}-\mathbf{x}_{\mathbf{2}} \cdot \mathbf{C}_{\mathbf{f}} \cdot \mathbf{x}_{\mathbf{f} 2}\right) \\
\mathbf{0}
\end{array}\right]
\end{aligned}
$$

the derivative of a Lyapunov function can be written where now the state, $\mathbf{x}$, in (4.3-3) would be replaced with the error, e. With this substitution, the expression would have the same terms as in (4.4-6) for what pertains to the first row, linear part, of (4.6-1) and only the nonlinear terms in the last row, nonlinear term, would have to be calculated separately. We obtain, using the same cross reference indices as in (4.4.-6), with the addition of two indices for the last term in (4.5-1), 


$$
\begin{aligned}
& \dot{V}_{c l}\left(\mathbf{e}_{\mathbf{x}}\right)=\frac{1}{2} \cdot \mathbf{e}_{\mathbf{x}}^{\mathbf{T}} \cdot\left[\left(\mathbf{P}_{\mathbf{x}} \cdot \mathbf{A}+\mathbf{A}^{T} \cdot \mathbf{P}_{\mathbf{x}}\right)_{\mathrm{I}}-F_{m} \cdot\left(\mathbf{P}_{\mathbf{x}} \cdot \mathbf{B} \cdot \mathbf{C}_{\mathbf{i}}+\mathbf{C}_{\mathbf{i}}^{\mathbf{T}} \cdot \mathbf{B}^{\mathbf{T}} \cdot \mathbf{P}_{\mathbf{x}}\right)_{I I}\right] \cdot \mathbf{e}_{\mathbf{x}}+\frac{1}{2} \cdot \mathbf{e}_{\mathbf{x}_{\mathrm{f}}}^{\mathbf{T}} \cdot\left(\mathbf{P}_{\mathbf{x}_{\mathbf{f}}} \cdot \mathbf{A}_{\mathbf{f}}+\mathbf{A}_{\mathbf{f}}^{\mathbf{T}} \cdot \mathbf{P}_{\mathbf{x}_{\mathrm{f}}}\right)_{\mathrm{III}} \cdot \mathbf{e}_{\mathbf{x}_{\mathbf{f}}}+ \\
& +\frac{1}{2} \cdot F_{m} \cdot \mathbf{e}_{\mathbf{x}}^{\mathbf{T}} \cdot\left(\mathbf{P}_{\mathbf{x}} \cdot \mathbf{B} \cdot \mathbf{C}_{\mathbf{f}}+\mathbf{C}_{\mathbf{f}}{ }^{\mathrm{T}} \cdot \mathbf{B}^{\mathrm{T}} \cdot \mathbf{P}_{\mathbf{x}}\right)_{\mathrm{IV}} \cdot \mathbf{e}_{\mathbf{x}_{\mathrm{f}}}+\frac{1}{2} \cdot \mathbf{e}_{\mathbf{x}_{\mathrm{f}}}^{\mathbf{T}} \cdot\left(\mathbf{P}_{\mathbf{x}_{\mathrm{f}}} \cdot\left[\begin{array}{ll}
\mathbf{0} & -\mathbf{B}_{\mathbf{f}}
\end{array}\right]+\left[\begin{array}{ll}
\mathbf{0} & -\mathbf{B}_{\mathbf{f}}
\end{array}\right]^{\mathrm{T}} \cdot \mathbf{P}_{\mathbf{x}_{\mathrm{f}}}\right)_{\mathrm{VI}} \cdot \mathbf{e}_{\mathbf{x}}+ \\
& +F_{m} \cdot \mathbf{e}_{\mathbf{x}}^{\mathbf{T}} \cdot \mathbf{P}_{\mathbf{x}} \cdot \Delta \mathbf{A} \cdot\left(\mathbf{x}_{\mathbf{2}} \cdot \mathbf{C}_{\mathbf{i}} \cdot\left(\mathbf{I}+\frac{D \cdot T_{s}}{2} \cdot \mathbf{A}_{\mathbf{1}}\right) \cdot \mathbf{x}_{\mathbf{2}}-\mathbf{x}_{\mathbf{1}} \cdot \mathbf{C}_{\mathbf{i}} \cdot\left(\mathbf{I}+\frac{D \cdot T_{s}}{2} \cdot \mathbf{A}_{\mathbf{1}}\right) \cdot \mathbf{x}_{\mathbf{1}}\right)_{\mathrm{IX}}+ \\
& +F_{m} \cdot \mathbf{e}_{\mathbf{x}}^{\mathbf{T}} \cdot \mathbf{P}_{\mathbf{x}} \cdot \Delta \mathbf{A} \cdot\left(\mathbf{x}_{1} \cdot \mathbf{C}_{\mathbf{f}} \cdot \mathbf{x}_{\mathbf{f} 1}-\mathbf{x}_{\mathbf{2}} \cdot \mathbf{C}_{\mathbf{f}} \cdot \mathbf{x}_{\mathbf{f} 2}\right)_{\mathrm{X}}-\left[\mathbf{e}_{\mathbf{x}}^{\mathbf{T}} \cdot \mathbf{P}_{\mathbf{x}} \cdot F_{m} \cdot(\mathbf{B}+\Delta \mathbf{A} \cdot \mathbf{x})_{\mathrm{VII}}+\left(\mathbf{e}_{\mathbf{x}_{\mathrm{f}}}^{\mathbf{T}} \cdot \mathbf{P}_{\mathbf{x}_{\mathrm{f}}} \cdot \mathbf{B}_{\mathbf{f}}\right)_{\mathrm{VIII}}\right] \cdot r
\end{aligned}
$$

or after expliciting the terms,

$$
\begin{aligned}
& \dot{V}_{c l}\left(\mathbf{e}_{\mathrm{x}}\right)=-\left.\frac{1}{R_{l d}} \cdot e_{x_{2}}^{2}\right|_{\mathrm{I}}-F_{m} \cdot V_{i n} \cdot M \cdot R_{i} \cdot\left(e_{x_{1}}^{2}-\frac{1}{D^{\prime} \cdot R_{l d}} \cdot e_{x_{1}} \cdot e_{x_{2}}\right)_{\mathrm{II}}+\left[-\left(1+\frac{C_{1}}{C_{3}}\right) \cdot \frac{1}{R_{2}} \cdot e_{x_{f 1}}^{2}-\frac{1}{2} \cdot \frac{1}{R_{2}} \cdot e_{x_{f 1}}^{2}+\frac{1}{2} \cdot \frac{1}{R_{2}} \cdot e_{x_{f 1}} \cdot e_{x_{f 2}}\right]_{\mathrm{III}} \\
& +F_{m} \cdot V_{i n} \cdot M \cdot \omega_{i} \cdot\left[\frac{1}{2} \cdot\left(e_{x_{1}} \cdot e_{x_{f 2}}+e_{x_{2}} \cdot e_{x_{f 1}}\right)-\frac{e_{x_{2}}}{D^{\prime} \cdot R_{l d}} \cdot e_{x_{f 2}}\right]_{\mathrm{IV}}-\left[\frac{1}{2} \cdot C_{1} \cdot\left(e_{x_{2}} \cdot e_{x_{f 1}}+e_{x_{1}} \cdot e_{x_{f 2}}\right)+C_{3} \cdot e_{x_{2}} \cdot e_{x_{f 2}}\right]_{\mathrm{VI}} \\
& -\left.F_{m} \cdot R_{i} \cdot\left(x_{11} \cdot x_{22}-x_{12} \cdot x_{21}\right) \cdot e_{x_{1}}\right|_{\mathrm{IX}}+\left.F_{m} \cdot \omega_{i} \cdot\left(x_{11} \cdot x_{22}-x_{12} \cdot x_{21}\right) \cdot e_{x_{f 2}}\right|_{\mathrm{X}}- \\
& -\left[F_{m} \cdot V_{i n} \cdot M \cdot\left(e_{x_{1}}-\frac{e_{x_{2}}}{D^{\prime}} \cdot \frac{1}{R_{l d}}\right)_{\mathrm{VII}}+F_{m} \cdot\left(e_{x_{1}} \cdot x_{22}-e_{x_{2}} \cdot x_{12}\right)_{\mathrm{VII}}+\left(C_{1} \cdot e_{x_{f 1}}+C_{3} \cdot e_{x_{f 2}}\right)_{\mathrm{VIII}}\right] \cdot r
\end{aligned}
$$

Neglecting the small terms, as in (4.3-8) we obtain

$$
\begin{aligned}
& \dot{V}_{c l}\left(\mathbf{e}_{\mathbf{x}}\right) \cong-\left.\frac{1}{R_{l d}} \cdot e_{x_{2}}^{2}\right|_{\mathrm{I}}-F_{m} \cdot V_{i n} \cdot M \cdot R_{i} \cdot\left(e_{x_{1}}^{2}-\frac{1}{D \cdot R_{l d}} \cdot e_{x_{1}} \cdot e_{x_{2}}\right)_{\mathrm{II}}-F_{m} \cdot\left[V_{i n} \cdot M \cdot\left(e_{x_{1}}-\frac{e_{x_{2}}}{D} \cdot \frac{1}{R_{l d}}\right)_{\mathrm{VII}}+\left(e_{x_{1}} \cdot x_{22}-e_{x_{2}} \cdot x_{12}\right)_{\mathrm{VII}}\right] \cdot r+ \\
& +F_{m} \cdot V_{i n} \cdot M \cdot \omega_{i} \cdot\left[\frac{1}{2} \cdot\left(e_{x_{1}} \cdot e_{x_{f 2}}+e_{x_{2}} \cdot e_{x_{f 1}}\right)-\frac{e_{x_{2}}}{D \cdot R_{l d}} \cdot e_{x_{f 2}}\right]_{\mathrm{IV}}-\left.F_{m} \cdot R_{i} \cdot x_{22} \cdot e_{x_{1}}^{2}\right|_{\mathrm{IX}}+\left.F_{m} \cdot \omega_{i} \cdot x_{22} \cdot e_{x_{1}} \cdot e_{x_{f 2}}\right|_{\mathrm{X}}
\end{aligned}
$$

where the terms "IX" and "X" have been approximated with the new expressions since, in practice, $x_{21} \cong x_{22}$, being the internal variables denoting each individual module voltage perturbation, Fig. 4.2 and Fig. 4.3, as given by the new modeling technique. These quantities are not accessible for physical measurements, but they greatly alleviate the modeling task. The following calculations will show that a practical control law can be inferred from an engineering perspective, based only on the variables that are available in the real system.

It can be readily seen that the first two terms in (4.5-4) are negative. The rest of the terms vary their signs, as the signs of the different error quantities vary. As expected and can be 
checked on practical examples simulations, the dominant factors within these terms are the ones containing the inductor currents error, $e_{x}$; the other ones can be omitted in a first approximation. This is convenient from an engineering standpoint, because each current is accessible for measurement, being actually used in PCMC. The product $\omega_{i} \cdot e_{x f 2}$ is nothing but the error in the outputs of the internal OpAmp compensators for each module, $\omega_{i} \cdot e_{x f 2}=e_{\text {vea }}$, also accessible for sensing.

If a control law would be derived for the " $r$ " input so the Lyapunov function derivative stays negative, it would need to cancel all the terms except the first two, for example. Based on the above remarks and using (4.5-4), we obtain.

$$
r \cong \frac{\frac{1}{2} \cdot \omega_{i} \cdot V_{i n} \cdot M \cdot e_{x_{f 2}} \cdot e_{x_{1}}-R_{i} \cdot x_{22} \cdot e_{x_{1}}^{2}+\omega_{i} \cdot x_{22} \cdot e_{x_{f 2}} \cdot e_{x_{1}}}{V_{i n} \cdot M \cdot e_{x_{1}}+x_{22} \cdot e_{x_{1}}},
$$

which can be simplified to

$$
r=\frac{\left(\frac{1}{2} \cdot V_{i n} \cdot M+x_{22}\right) \cdot e_{v_{c a}}-R_{i} \cdot x_{22} \cdot e_{x_{1}}}{V_{i n} \cdot M+x_{22}} .
$$

Observing also that $x_{21}$ and $x_{22}$ being the internal, output, voltage variation for each converter they are approximately half of the system's output voltage perturbation, $x_{21} \cong x_{22} \cong v_{o} / 2$ and using the fact that $V_{o d}=V_{\text {in }} \cdot M$ is the ideal, desired, DC output voltage, we have that

$$
r=\frac{\frac{1}{2} \cdot\left(V_{o d}+v_{o}\right)}{V_{o d}+v_{o} / 2} \cdot e_{v_{e a}}-R_{i} \cdot \frac{\frac{1}{2} \cdot v_{o}}{V_{o d}+\frac{1}{2} \cdot v_{o}} \cdot e_{x_{1}} \cdot
$$

Adding an adjustable gain factor for each term and since in the real system it is easier to sense the total, DC plus AC, output voltage, $v_{o_{-} \text {tot }}=V_{o d}+v_{o}$ we can further simplify (4.5-7) as

$$
r=K_{1} \cdot \frac{v_{o_{-} t o t}}{v_{o_{-} t o t}+V_{o d}} \cdot e_{v_{e a}}-K_{2} \cdot R_{i} \cdot \frac{v_{o_{-} t o t}-V_{o d}}{v_{o_{-} t o t}+V_{o d}} \cdot e_{x_{1}} .
$$

Even expression (4.5-8) can be further approximated in terms of its computation since the main requirement is to feed back the two error quantities, mainly the error in the two currents, with their respective adjustable gains. We obtain, acquainting for the last term sign change, 


$$
r=K_{1} \cdot e_{v_{e a}}+K_{2} \cdot R_{i} \cdot \frac{v_{o_{-} t o t}}{V_{o d}} \cdot e_{x_{1}}
$$

Equations (4.5-8) and (4.5-9) have been obtained after many simplifications due to the complicated, implicit form, of the error equation in the case of non-identical converters. Even in this case, implementing the exact control law that would cancel all the variable sign terms is not feasible in practice and the simulations run in several instances also shown computational issues. However, with a few gain adjustments, (4.5-8) and (4.5-9) can be used as a Lyapunov supervisory controller for the fuzzy logic one, if the latter has a high gain that brings the system unstable. One implementation is to sum the two controllers action, either continuously or at a specified instant, if the current sharing error does not go within the desired boundaries. The commutation indicator function, $I$, can be done through a direct switching action, or employing a soft switch-in step, [36],

$$
u_{t o t}=u_{f u z z y}+I \cdot u_{L y a p}
$$

with the indicator function, $I=1$ if $|\mathbf{x}|$ or $\left|e_{i o}\right|$ is greater than the desired boundary $M$ and zero otherwise.

If used stand alone, the last two control laws would not offer the performance of the fuzzy controller, being inferred after a lot of simplifications and dynamic terms cancellations, as mentioned previously. Because of the dynamic, derivative, terms simplifications, for boost derived converters, as in this example, the lag effect of the RHPZ in the power stage has to also be considered when choosing the two gains, $K_{1}$ and $K_{2}$. For example, when a fast fuzzy controller is used, $e_{x l}$ will be different instantaneously than $e_{i o}$, having opposite signs since the inductor current will briefly lag the output one. For a stand alone, slow, Lyapunov controller, the two would have the same sign. In addition and linked to the inductor currents error, $e_{x l}$, the error in the outputs of the internal voltage compensators, $e_{v e a}$, may be slightly shifted with opposite signs, not only because of the RHPZ dynamic effect but mainly because of the differences between the values of the converter's parasitics and components, e.g. tolerances, offsets, etc. For substantially different converters the sign of $K_{l}$ may thus need to be adjusted when employing this simplified supervisory control law. The two current's error will always need to be fed back, even though only a voltage mode controller is employed; this is fairly obvious from an 
engineering viewpoint even though the simplified relations (4.5-8) and (4.5-9) may suggest that if PCMC is not used, the term in $e_{x 1}$ will not be present. This is only because of the simplifying assumptions and term reductions in the two expressions and hence all the terms required in practice need to be kept in. Also, one has to recall that we had to write the equations and come up with the models and control laws considering the entire state error, which is more stringent and complicated than considering only the current sharing error. This was dictated by the complicated nature of the system, as the only access path to the current sharing error quantity in a modern control systems framework.

To recapitulate, even after the simplifications required by the complicated form of this type of systems, we were able to come up with a control strategy that offers good bounds for the converter variables. The control rule is intuitive engineering wise, since it practically feeds back opposite sign information proportional with the currents error and with the difference of the two DC-DC individual modules inner control quantities, which is expected for two plants tracking each other. One of the features that complicates the system structure is that the presently established control schemes for the DC-DC converters in the industry are not a linear state feedback, per se. They are a summation of different loops for each state variables, with different gains and/or OpAmp compensators in each loop. This makes the models to come out in a cumbersome form for direct modern control implementations, especially in parallel, distributed power systems. One direction would be to have a linear, stable, full state feedback for each module, which would then make the plants more amenable to be integrated in a paralleled system, where the same control strategy can be continued. Another approach is to recognize the variable structure features of the DC-DC topologies and to employ a sliding mode controller, [13], [19], [36], in each module. Associated to that or independent, control laws through inputoutput linearization and coordinate transformations, [13], [20], can also be tried. The fuzzy logic interface is described in the next section.

As a last, but important note, an additional stabilizable controller will only be needed if a very high gain fuzzy controller is desired. This is because, due to the internal structure of the fuzzy logic compensator described in Chapter 2, when the error is at maximum, the control output will saturate Negative Big or Positive Big and if we do not use a very high gain, we would have the system's response to a constant command on the DC-DC module reference input; this may be slower but it is stable in almost all cases. 


\section{Chapter 5}

\section{FUZZY LOGIC CONTROL OF PARALLELED DC/DC CONVERTER MODULES}

Based on the modern control systems approach introduced in Chapter 2 through Chapter 4, this chapter introduces and elaborates fuzzy logic control for parallel converter systems, with the objective of deriving a new, improved, current sharing control method for master - slave type systems.

\subsection{Fuzzy Logic Controller Design using a PID Compensator as Expert}

Present control schemes make use of a PI or of a lead-lag with integrator controller for the active current sharing loop, [25]-[30],

$$
H(s)=\omega_{i} \cdot \frac{1}{s} \cdot \frac{s+\omega_{z}}{s+\omega_{p}} .
$$

Figure 5.1-1 shows the generic MSC scheme with converter \#1 as master.

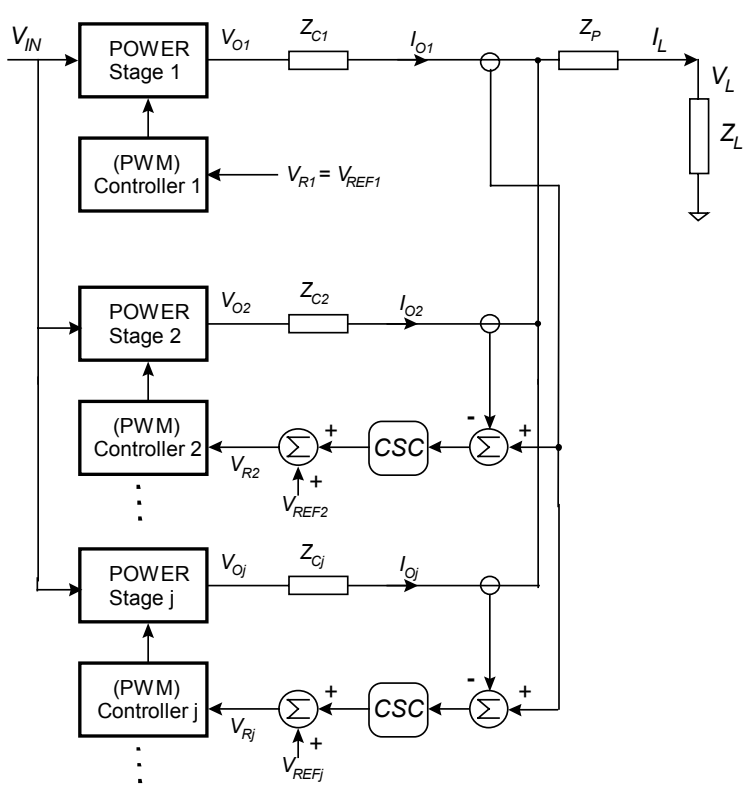

Figure 5.1-1 - Control scheme used for paralleling $N$ converter modules with MSC 
A common CSC that uses a lead-lag cell plus an integrator, is shown in

Figure 5.1-2, below.

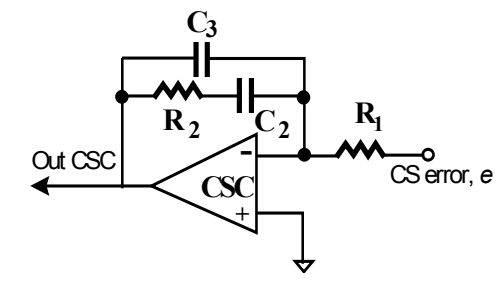

Figure 5.1-2 - Classical CSC architecture

Due to the highly interconnected system, complex modeling does not provide a practical engineering way to consider both dynamic response and stability, the latter being analyzed more in the literature by using linear analysis simplifications. For improved transient performance, fuzzy control appears to have a strong potential. Present implementations can also be regarded as educated heuristic approaches since even after some analysis, they still use an iterative simulation-experimentation process to choose a final, stable, design, [19]- [21]. In this line of thinking, one can notice that a first improvement that can be done is to upgrade the lead-lag plus integrator controller in Figure 5.1-2 to a PID one because of its generality and tuning capability. This offers the designer a good model or expert system that can be used to derive the values of part of the Fuzzy Logic Controller (FLC), as it will be shown below. The PID controller also offers a higher Bandwidth (BW) while retaining sufficient Phase Margin (PM). The proposed PID compensator is described by the transfer function

$$
H(s)=k_{p}+\frac{k_{i}}{s}+k_{d} \cdot \frac{s}{s+10 \cdot \omega_{z E S R}} .
$$

where the derivative realizability pole has been chosen high enough, i.e., ten times the frequency of the filtering capacitor ESR zero, which is one of the highest frequencies of the DC/DC converter; depending on the application, half the switching frequency may be used, if this is higher than the ESR zero frequency. Comparing (5.1-1) and (5.1-2) one can see more clearly, now, that the PID structure offers access into each of the Proportional, Integral and Derivative components separately and hence the capability of quantifying each individual action in deriving the values for a FLC. 
The PID improved results over the classical, OpAmp, controller will be used as the expert system in developing the consequents values, in the Inference rules section of the FLC. Figure 5.1-3 shows the block diagram of a generic FLC, which uses the current share error, $e$, into a PD fuzzy scheme combined with an integral component needed for the zero steady state error, [2].

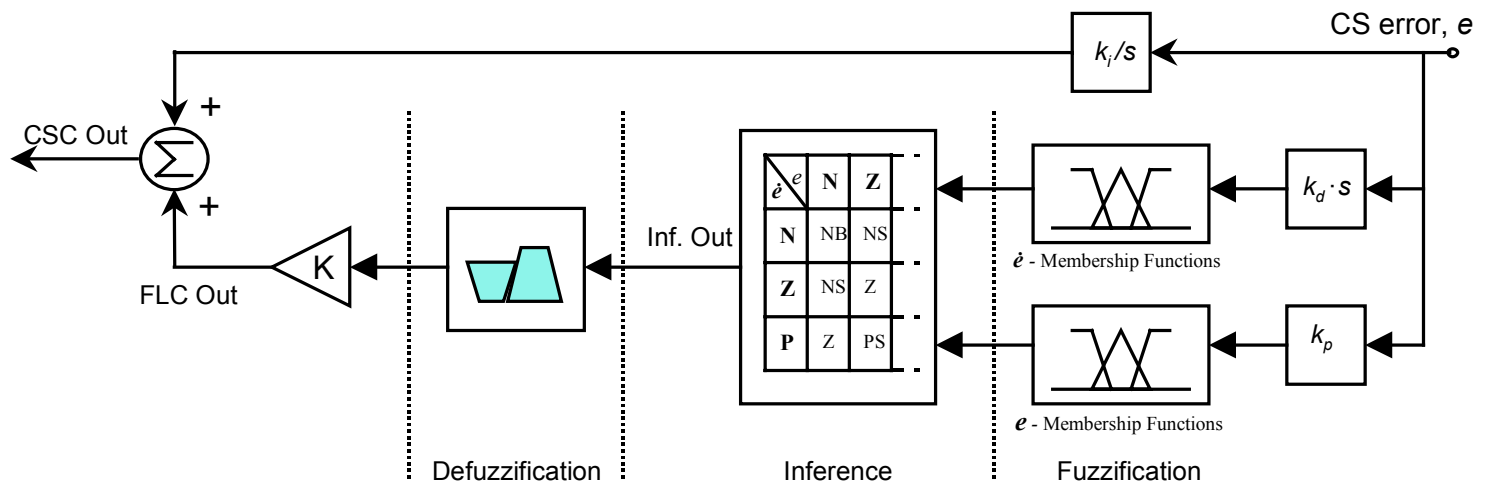

Figure 5.1-3 - Fuzzy logic implementation of CSC

A three range partition, negative $(\mathrm{N})$, zero $(\mathrm{Z})$ and positive $(\mathrm{P})$, can be chosen for the inputs, $e$ and $\dot{e}$. A more refined five-range partition, negative-big (NB), negative-small (NS), zero (Z), positive-small (PS) and positive-big (PB) is used for the output. Based on the usual action of a PD cell, by looking at its output range as a function of the input range from (2), a rule-based inference is chosen for each consequent; the membership functions and respective rules are shown in Figure 5.1-4.
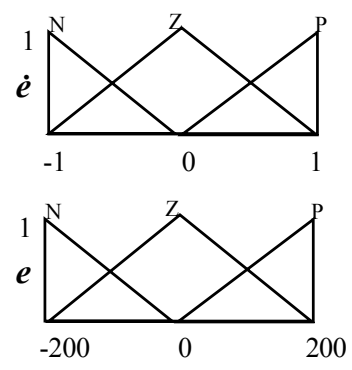

\begin{tabular}{|c|c|c|c|}
\hline$\dot{e} \backslash e$ & \multicolumn{1}{|c|}{$\mathbf{N}$} & \multicolumn{1}{|c|}{$\mathbf{Z}$} & \multicolumn{1}{|c|}{$\mathbf{P}$} \\
\hline $\mathbf{N}$ & $\mathrm{NB}(-40)$ & $\mathrm{NS}(-20)$ & $\mathrm{Z}(0)$ \\
\hline $\mathbf{Z}$ & $\mathrm{NS}(-20)$ & $\mathrm{Z}(0)$ & $\mathrm{PS}(20)$ \\
\hline $\mathbf{P}$ & $\mathrm{Z}(0)$ & $\mathrm{PS}(20)$ & $\mathrm{PB}(40)$ \\
\hline
\end{tabular}

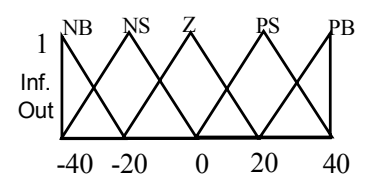

Figure 5.1-4 - Membership functions and rule-based inference for the fuzzy logic controller in Figure 5.1-3 
We employ a product inference engine, singleton fuzzifier and center average defuzzifier, [36], [Appendix A]. The membership functions are triangular, laid out symmetrical within the ranges for each variable's universe of discourse, i.e., $e, \dot{e}$ and the output of the inference section, respectively, Figure 5.1-3, and Figure 5.1-4. An example of establishing the fuzzy rule values based on the PID expert in (5.1-2) is as follows. Assume that after upgrading the OpAmp compensator to a PID one, we determined through simulations, for example, that the ratio between the gains of the proportional and derivative, $k_{p} / k_{d}$, part is 200 . Therefore, for a range of $e$ between -1 and 1, we would need to consider a range between -200 and 200 for $\dot{e}$. Now, if $e$ is $\mathrm{N}(-1)$ and $\dot{e}$ is $\mathrm{N}(-200)$, then the PID would roughly give a control value of -40 , hence the value NB of -40 for the consequent.. The other rules are inferred similarly. The gain block $K$ is the general gain used for the output of the FLC, being adjusted depending on each application case.

Further improvement in partitioning the input spaces as well as using neuro-fuzzy techniques in an adaptive grid of controllers, [24]-[25], can be considered. The IF-THEN rules and the shape of the membership functions can be generated and optimized using several numeric methods or genetic algorithms procedures. In the latter case, one can infer the desired fitness functions from simulation results run considering the detailed system's circuitry in the conditions that are of interest for each specific application.

As an important note, the PID controller is not actually used as a design goal, it is just an intermediary phase, as an expert system, in developing the FLC. Thus, his parameters, even though important, are not of critical exact value but rather a good guideline for the fuzzy logic system. Two examples will be presented, for the buck and respectively boost topologies. The first case employs the same numerical values above, as it will be briefly detailed next. The second case, for a PCMC boost converter system, will be treated with extended simulations that will consider also a Lyapunov supervisory controller. The results are given in the next chapter, after a Lyapunov adaptation law is linked to the concepts of the fuzzy logic controller, in the next section. 


\subsubsection{Classical versus Fuzzy Logic Control for Active Current Sharing}

This section demonstrates the implementation of a master-slave control fuzzy current sharing controller with improved performance over a solidly developed classical design, such as in [27], using the concepts introduced above.

The small signal loop gain used in a three voltage mode Buck converter system employing the MSC topology in Figure 3.1-1 and CSC in Figure 5.1-2, is reproduced in Figure 5.1-5 together with the PID improved loop gain. The system is supplied from $12 \mathrm{~V}$ input voltage, delivers a $5 \mathrm{~V}$ output voltage at $15 \mathrm{~A}$ load for each converter, thus $45 \mathrm{~A}$ nominal load for the whole system and was simulated using Pspice. The bandwidth of the classical CSC is 29 $\mathrm{KHz}$, with a $\mathrm{PM}$ of $47^{\circ}$ and a Gain Margin (GM) of $14 \mathrm{~dB}$. Its transfer function is:

$$
H(s)=\omega_{i} \cdot \frac{1}{s} \cdot \frac{1+\frac{s}{\omega_{z}}}{1+\frac{s}{\omega_{p}}},
$$

with $\omega_{i}=\frac{1}{R_{1} \cdot\left(C_{2}+C_{3}\right)}=1200 \mathrm{sec}^{-1}, \omega_{z}=\frac{1}{R_{2} \cdot C_{2}}=2 \cdot \pi \cdot 50 \mathrm{sec}^{-1}$ and $\omega_{p}=\frac{C_{2}+C_{3}}{R_{2} \cdot C_{2} \cdot C_{3}}=2 \cdot \pi \cdot 5 \cdot 10^{4} \mathrm{sec}^{-1}$. The PID design has been adjusted for a $\mathrm{BW}$ of $80 \mathrm{KHz}$, a reasonable trade-off between noise immunity and speed, with a PM also of $47^{\circ}$ and infinite GM. 


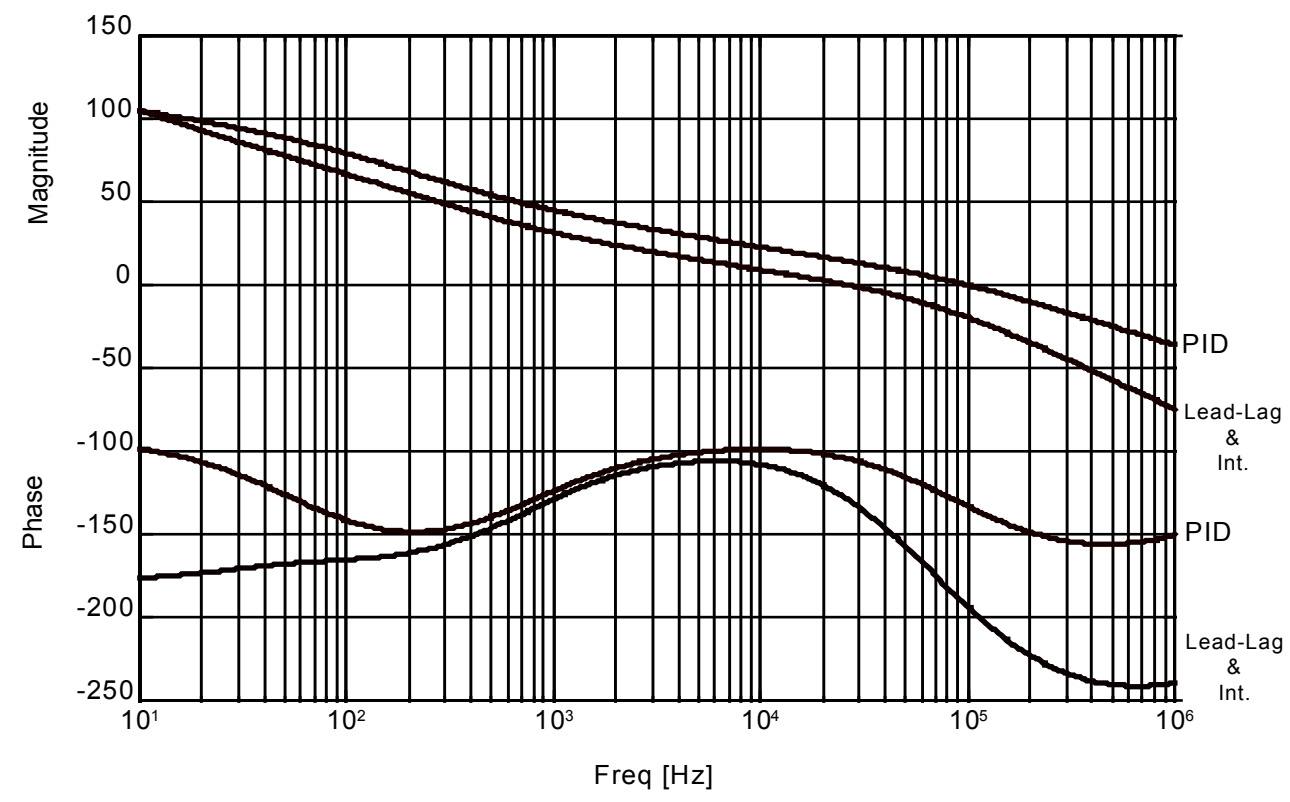

Figure 5.1-5 - Small signal loop gains for a classical design and improved PID design

The PID controller has $k_{p}=20, k_{i}=1$ and $k_{d}=0.1$, which give the same numerical values used to describe the generic FLC design methodology above, [2]. From Figure 5.1-5, a better performance of the PID controller can be inferred, first small-signal wise and, as it will be verified next, also in the time domain; it will be also shown that the FLC gives the best performance of all three control methods.

Time domain simulations have been done on the previous mentioned system where one module has the nominal parameters - reference voltage, output filter values, internal compensator $\mathrm{R}$ and $\mathrm{C}$ values- and the parameters of the other two deviate from nominal within usual fabrication tolerances for these components. Comparative 50\% and $90 \%$ nominal load step responses are given in Figure 5.1-6 for the classical design in [27], Figure 5.1-6 (a), the PID design, Figure 5.1-6 (b) and the fuzzy controller, Figure 5.1-6 (c). 

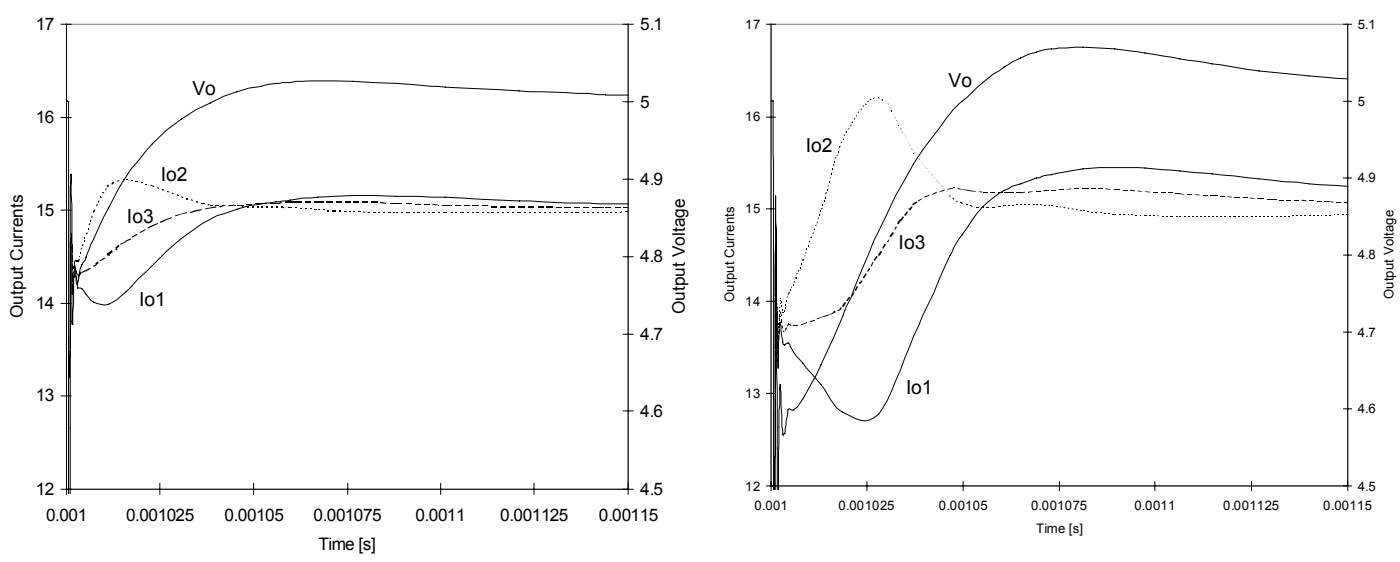

(a)
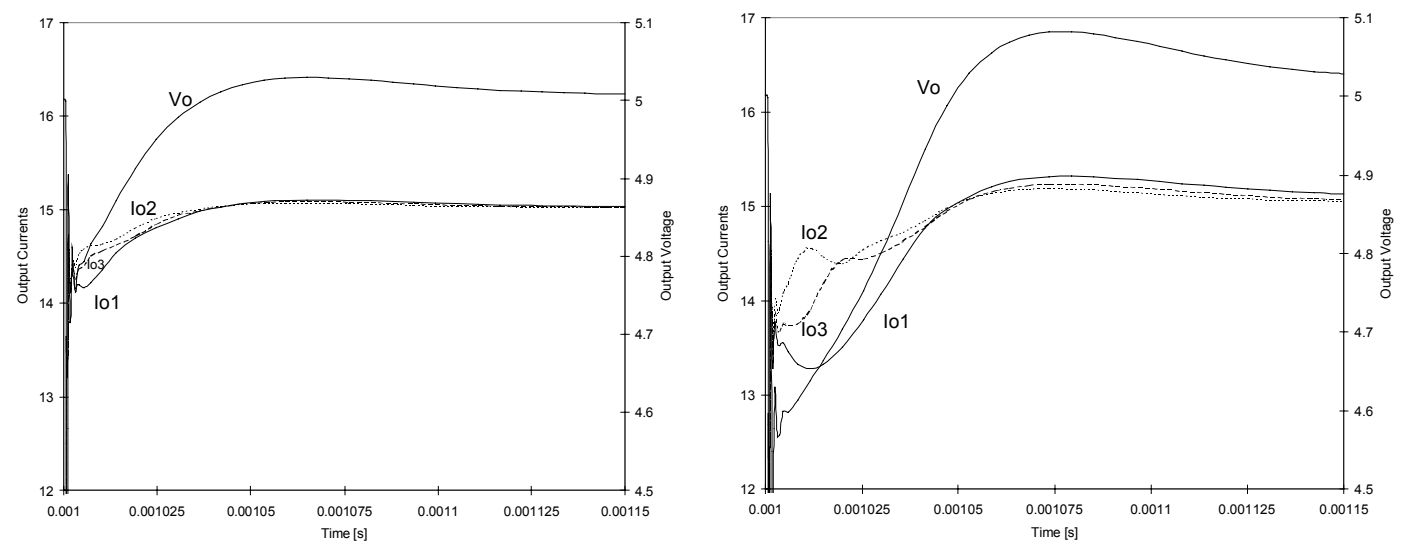

(b)
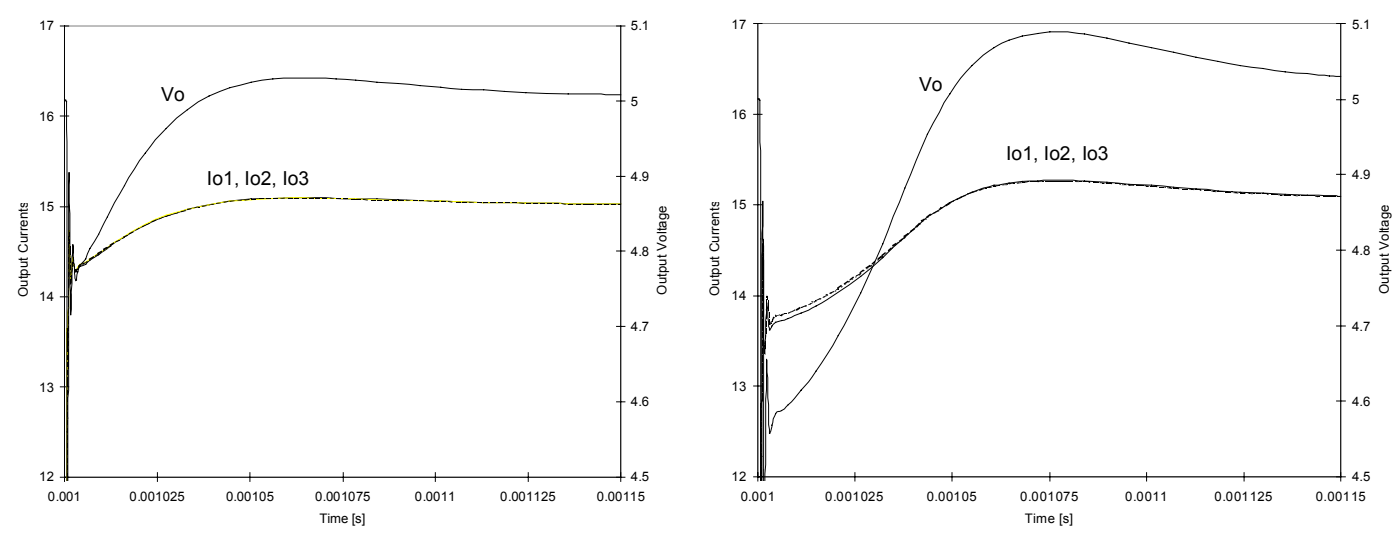

(c)

Figure 5.1-6 - Step load responses for 50\% and 90\% of the nominal load: (a)-System with the classical CSC in Figure 5.1-2; (b)-System employing a PID CSC; (c)-System using a Fuzzy Logic CSC

A substantial improvement in the transient performance is noted in the case of the PID and especially of the fuzzy logic controller. Almost identical responses for the two step load cases when using the fuzzy control approach, shows the validity of fuzzy control in dealing naturally with the nonlinear character of the system. The low performance index in the traditional cases is due to the fact that a conventional linear controller, designed only on a small 
signal model, is valid only near the operating point and not for the entire plant trajectory. It can also be noticed that for each step load, the voltage waveforms for the three controllers are very close since, in general, the output voltage dynamics are influenced only by the internal loops of the master converter, the slave converters exhibiting practically no voltage gain.

Figure 5.1-7 depicts the averaged duty cycle plots for the same cases shown in Figure $5.1-6$
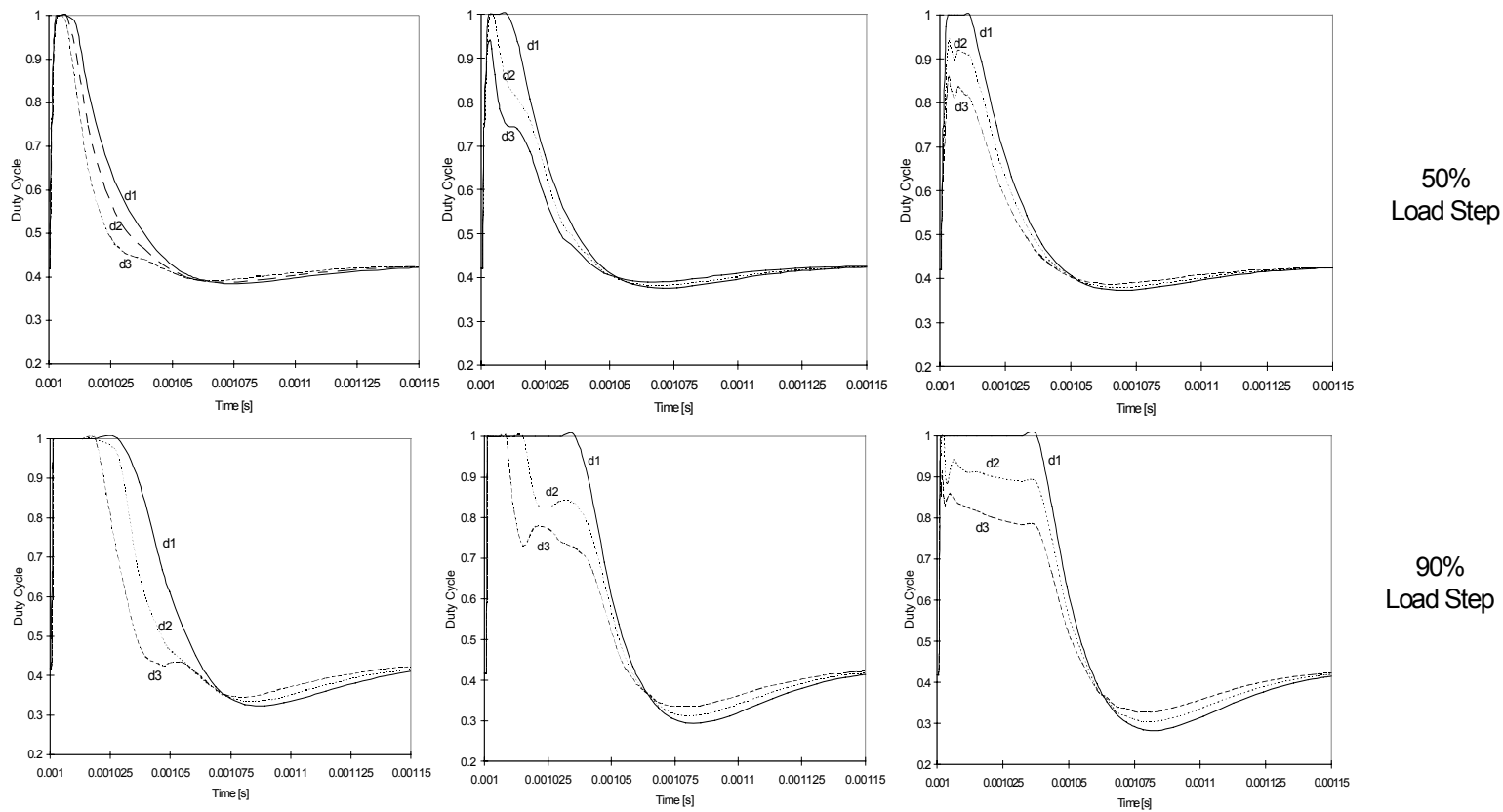

$90 \%$
Load Step

(a)

(b)

(c)

Figure 5.1-7 - CSC Control Effort (averaged duty cycles): (a)-With the classical CSC in Figure 5.1-6;

(b)-Employing a PID CSC; (c)-Using a Fuzzy Logic CSC

One can again see that the fuzzy controller gives the minimal needed effort to control the system current sharing. The duty cycle of the two slave converters, $d_{2}$ and $d_{3}$, never saturate in this case, Figure 5.1-7 (c). In comparison, the use of the compensator in Figure 5.1-2 (a), is the slowest and requires the most effort. In all cases the master duty cycle saturates for a certain period since the master does not get back any information about current sharing and hence tries to use all its control range for output voltage regulation, as mentioned above. 


\subsection{On a Lyapunov stable Fuzzy Logic Control}

This paragraph highlights the feasibility of a stable Lyapunov adaptive control when employing the fuzzy logic controller above, provided that certain conditions are met by the system's parameters. The algorithm, which proposes an adaptation mechanism for the fuzzy membership functions on a Lyapunov trajectory, has the merit of proving the existence of such a control law under a set of constraints that will be detailed below. It is very general, however and it does not offer a direct implementation since a high degree of trial and error gain adjustments is needed in order to see any results, for each application. However, based on its existence, a Lyapunov supervisory control can be tried considering the specific plant in each practical case. For this study, the Lyapunov controller developed in paragraph 4.3, equations (4.5-8) - (4.5-10) will be used. The results are given next.

A good way to highlight the CS error is to employ an input-output linearization, [20], [36] - [38], so the reference input is linearly present in the expression of the output, which is the second component of the state vector, $x_{2}$, as detailed in the previous sections. The general model of a system in this form is

$$
x^{(n)}=f(x)+g(x) \cdot u
$$

Equation (4.3-1), describing the closed loop plant, can be put in the form (5.2-1) for the desired output and we would obtain systems of different orders and relative degrees, depending on each specific DC-DC topology that is analyzed. For the generality of the proof, we consider a case when $n$ derivations are needed.

From equation (5.2-1), if we could easily implement the ideal control,

$$
u_{i d}=\frac{1}{g(\mathbf{x})} \cdot\left[-f(\mathbf{x})+y_{r}^{(n)}+\mathbf{k}^{T} \cdot \mathbf{e}\right]
$$

for tracking the reference $y_{r}$ and hence canceling the error $e=y_{r}-y$, one can take $\mathbf{k}=\left[k_{n}, \ldots, k_{l}\right]^{\mathrm{T}}$ such that all roots of the polynomial $s^{n}+k_{1} s^{n-1}+\ldots+k_{n}$. are in the open left-half complex plane. Then, the closed-loop system becomes 


$$
e^{(n)}+k_{1} \cdot e^{(n-1)}+\cdots+k_{n} \cdot e=0
$$

Because of the way we chose $\mathbf{k}$, we would have that $e(t) \rightarrow 0$ as $t \rightarrow \infty$, i.e., the plant output $y$ tracks the reference $y_{r}$ asymptotically and the poles can be placed so we get the desired transient response.

Since $f(\boldsymbol{x})$ and $g(\boldsymbol{x})$ are too complicated to use for deriving the ideal control in (5.2-2), or not known in detail, one can use the averaged model behavior to heuristically come up with the fuzzy controller. We use an easy to implement fuzzy logic control using product inference engine, $\boldsymbol{\xi}$, singleton fuzzifier, $\boldsymbol{\theta}$ and center average defuzzifier, $u_{f z}(\mathbf{e} \mid \boldsymbol{\theta})=\boldsymbol{\theta}^{\mathbf{T}} \cdot \boldsymbol{\xi}(\mathbf{e}),[36]$, [Appendix A]. Denoting with $\theta *$ the optimal singletons after the Lyapunov stability adaptation, and defining the minimum approximation error $w=u_{f z}\left(\mathbf{e} \mid \boldsymbol{\theta}^{*}\right)-u_{i d}$, we obtain after some algebra,

$$
\dot{\mathbf{e}}=\boldsymbol{\Lambda} \cdot \mathbf{e}+\mathbf{b} \cdot(\boldsymbol{\theta} *-\boldsymbol{\theta}) \cdot \boldsymbol{\xi}-\mathbf{b} \cdot w
$$

where $\mathbf{e}=\left[e, e, \ldots, e^{(n-1)}\right]^{\mathrm{T}}, \Lambda$ has the controllable canonical form with the elements of $\mathbf{k}$ as coefficients and $\mathbf{b}$ is a column matrix with the last element $b$ and the rest zero.

Consider now the Lyapunov function candidate

$$
V=\frac{1}{2} \cdot \mathbf{e}^{T} \cdot \mathbf{P} \cdot \mathbf{e}+\frac{b}{2 \cdot \gamma} \cdot(\boldsymbol{\theta} *-\boldsymbol{\theta})^{T} \cdot(\boldsymbol{\theta} *-\boldsymbol{\theta})
$$

with $\mathbf{P}$ satisfying the Lyapunov equation $\boldsymbol{\Lambda}^{\mathbf{T}} \cdot \mathbf{P}+\cdot \mathbf{P} \cdot \boldsymbol{\Lambda}=-\mathbf{Q}$, where $\mathbf{Q}$ and $\mathbf{P}$ are positive definite. We obtain

$$
\dot{V}=-\frac{1}{2} \cdot \mathbf{e}^{T} \cdot \mathbf{Q} \cdot \mathbf{e}-\mathbf{e}^{T} \cdot \mathbf{p}_{\mathbf{n}} \cdot b \cdot w+\frac{b}{\gamma} \cdot(\boldsymbol{\theta} *-\boldsymbol{\theta})^{T} \cdot\left(\gamma \cdot \mathbf{e}^{T} \cdot \mathbf{p}_{\mathbf{n}} \cdot \boldsymbol{\xi}-\dot{\boldsymbol{\theta}}\right)
$$

where $p_{n}$ is the last column of the $\mathbf{P}$ matrix. Therefore, if we choose the generic adaptation law

$$
\dot{\boldsymbol{\theta}}=\gamma \cdot \mathbf{e}^{T} \cdot \mathbf{p}_{\mathbf{n}} \cdot \boldsymbol{\xi}(\mathbf{x})
$$

then the last term in (5.2-6) is canceled and if we design the fuzzy controller with proper rules from the knowledge we have about the system, then the error $w$ will be small enough so the first 
term in (5.2-6) prevails and $V^{\prime}$ will be negative. Even though intuitively appealing, this type of control will not unconditionally satisfy all parameters boundedness and convergence performance requirements for any given plant. The following theorem, [36], shows that if the fuzzy control system is stable, then, under mild conditions, the tracking error will converge to zero.

Theorem 5.2-1: $\quad$ Consider the fuzzy logic control using product inference engine, $\xi$ singleton fuzzifier, $\theta$ and center average defuzzifier, $u_{f z}(\mathbf{e} \mid \boldsymbol{\theta})=\boldsymbol{\theta}^{\mathrm{T}} \cdot \boldsymbol{\xi}(\mathbf{e})$, employed above. If the state $\mathbf{x}$, the parameters $\theta, \xi$ and the minimum approximation error, $w$, are bounded, then:

a) The tracking error satisfies

$$
\int_{0}^{t}|\mathbf{e}(\tau)|^{2} d \tau \leq a+b \cdot \int_{0}^{t}|w(\tau)|^{2} d \tau
$$

where $a$ and $b$ are constants.

b) If $w$ is squared integrable, i.e., if $\int_{0}^{\infty}|w(t)|^{2} d t<\infty$, then $\lim _{t \rightarrow \infty}|\mathbf{e}(t)|=0$.

Therefore, the two key problems are (i) how to guarantee the boundedness of all the salient variables and parameters and (ii) how to make the minimum approximation error squared integrable. Since the fuzzy systems are universal approximators, we can make the minimum approximation error arbitrarily small by using more rules to construct the fuzzy system and hence the latter issue is solved. Issue (i) remains thus the main one. This can be solved by appending a supervisory control on top of our desired fuzzy logic one. Assume that we can determine state dependent bounds, which can be fairly loose for this type of applications, such that $|f(\mathbf{x})| \leq f^{\mathrm{U}}(\mathbf{x})$ and $0<g(\mathbf{x}) \leq g^{\mathrm{U}}(\mathbf{x})<b$. This is always a very reasonable assumption in $\mathrm{DC} / \mathrm{DC}$ converter systems due to the inherent saturation of the OpAmps and current limiting circuitry to their housekeeping supply levels. In these conditions, reference [36] shows how a stabilizable supervisory controller, $u_{s}$, based on the above limits, can be calculated and then appended to the existing FLC, similar to equation (4.5-10), so it intervenes and drives the derivative of the Lyapunov function negative when the system tends to be unstable. 


\section{Chapter 6}

\section{SIMULATED RESULTS Of FUZZY LOGIC CONTROLLED PARALLELED DC/DC CONVERTERS}

This chapter shows the implementation and simulated results for fuzzy logic control of three parallel DC/DC boost PCMC converters, designed based on the concepts introduced and analyzed in all previous chapters. The results show both a system without a stabilizable controller and one that uses a Lyapunov supervisory control law, applied when the gains of the fuzzy controller are chosen too high.

\subsection{Individual Converter Module Design Analysis}

The DC/DC converter used in the simulation is a PCMC boost converter that is supplied from $28 \mathrm{~V}$ input voltage and delivers a $44 \mathrm{~V}$ output voltage at $22 \mathrm{~A}$ load; these used to be traditional aerospace bus lines applications. The converter was originally used as a VA Tech school project, intended to include a challenging enough topology and design case, which has been upgraded in this study. The modules are chosen with relevant parasitics and tolerances, as listed below, so the effect of the unmatched parameters is present, which is even more important in the paralleled system. Their nominal values are $f_{s}=200 \mathrm{KHz}, L=10 \mu \mathrm{H}, R_{L}=20 \mathrm{~m} \Omega, C=500 \mu \mathrm{F}$, $R_{C}=10 \mathrm{~m} \Omega$. The internal loop compensator has the same architecture as the CSC in Figure 5.1-2 and, after design, has the parameters: $\omega_{i}=6.5876 \cdot 10^{3} \mathrm{rad} / \mathrm{s}, \omega_{z}=1.8519 \cdot 10^{3} \mathrm{rad} / \mathrm{s}, \omega_{p}=85.185 \cdot 10^{3}$ $\mathrm{rad} / \mathrm{s}$. In the large signal simulations, the OpAmp compensators are assumed supplied from $12 \mathrm{~V}$ and operate around a reference voltage of $5.1 \mathrm{~V}$.

Figure 6.1-1 shows the outer and overall loop gains, $T_{1}$ and $T_{2}$, [9]; $T_{2}$ would correspond to the " $G \cdot H$ " gain, used in classical control theory. 
T1 and T2 for the Master Converter

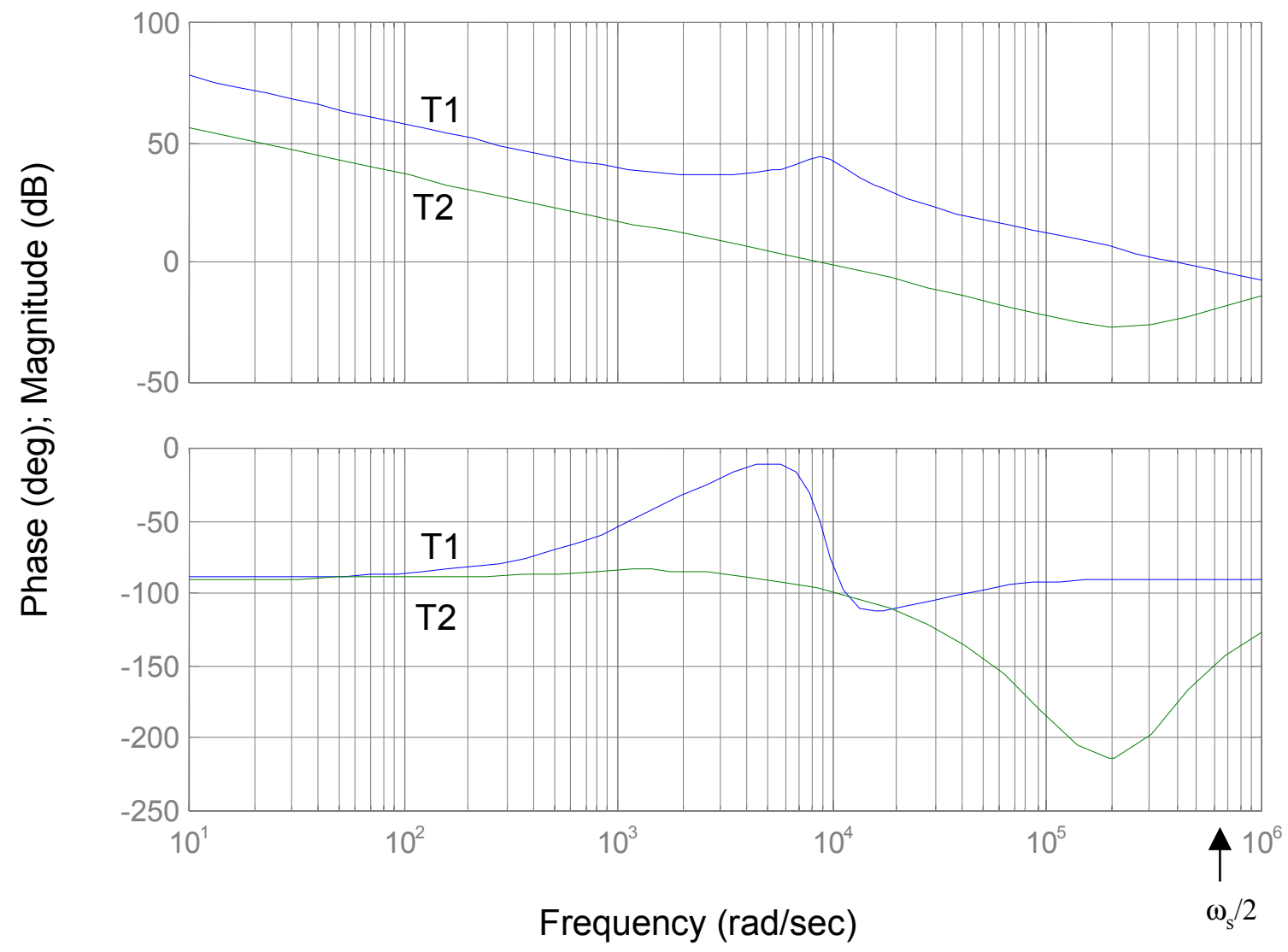

Figure 6.1-1 - Individual Module Loop Gains

As mentioned in [9], both loop gains contain useful information and can be used to verify a converter design and no gain alone gives all the detailed physical insight in the system's stability and time domain response. For detailed module analysis, reference [9] can be studied at length. Since our objective is the parallel system, only the main results will be investigated in order to make sure that we have a good, stable converter module when we integrate it in the distributed system. The overall loop gain, which is the sum of the current loop and voltage loop gains, $T_{I}=T_{i}+T_{v}$, has a crossover frequency of approximately $60 \mathrm{KHz}$ and a $\mathrm{PM}$ of approximately $85^{\circ}$. Its high BW (the switching frequency is $200 \mathrm{KHZ}$ ) verifies a good design in terms of having a high $\mathrm{BW}$ for the current loop gain, $T_{i}$, given in turn by the duty-cycle-to-inductor transfer function, which dominates the overall loop towards its crossover frequency, giving it almost a $90^{\circ}$ of PM, [9]. The "outer loop" gain, $T_{2}=T_{v} /\left(1+T_{i}\right)$, which is similar to a " $G H$ " gain, directly describes the system stability. It can be seen that $T_{2}$ has a BW of approximately $1.4 \mathrm{KHz}$ with a 
$\mathrm{PM}$ of $80^{\circ}$ and a GM of $20 \mathrm{~dB}$, which points to very good small signal stability. The low BW, large PM is typical for the boost topology design so it compensates for the RHPZ effect. In addition, in what concerns this study, it was chosen so the converter does not exhibit a strongly under-damped, oscillatory, response for low to moderate step load disturbances that are out of the small signal perturbation range, offering thus a good large signal behavior. The effect of the boost topology RHPZ, $\omega_{R H P Z}=81 \cdot 10^{3} \mathrm{rad} / \mathrm{s} \cong 12.9 \mathrm{KHz}$, can be briefly seen in the high frequency range, but it is overwritten by the effect of half the switching frequency. This is where the current loop sample and data effect, given by $H_{e}(s)$, takes over with its RHPPs since, through $T_{i}$, the RHP roots of $H_{e}(s)$ appear in the denominator of $T_{2}$.

\subsection{Paralleled System Results}

Small and large signal simulations have been done on a three PCMC boost converters system employing the modules described in the previous paragraph. The system delivers a $22 \mathrm{~A}$ load for each converter, thus $66 \mathrm{~A}$ nominal load for the system. The master converter module has the nominal parameters - reference voltage, output filter values, internal compensator $\mathrm{R}$ and $\mathrm{C}$ values - and the parameters of the other two deviate from nominal within the usual $1 \%$ to $10 \%$ fabrication tolerances. The filter components, L, C and their parasitic ESRs usually exhibit wider value tolerances. The OpAmps supply voltages, their $\mathrm{R}$ and $\mathrm{C}$ components in the compensation networks, as well as the DC reference voltage setpoints are varied at the lower ends since tighter specs can be ensured with the present technologies.

The system small signal loop gains employing the MSC topology in Figure 5.1-1 are plotted in Figure 6.2-1 together with the PID improved loop gain. The parameters of the PID compensators are $k_{p}=2, k_{i}=1, k_{d}=0.2$ for the compensator controlling the Master (converter \#1) and Slave \#1 (converter \#2) pair and $k_{p}=1.5, k_{i}=1, k_{d}=0.15$ for the controlling the Master and Slave \#2 (converter \#3) pair. 
Outer Loop Gains for the Paralleled System

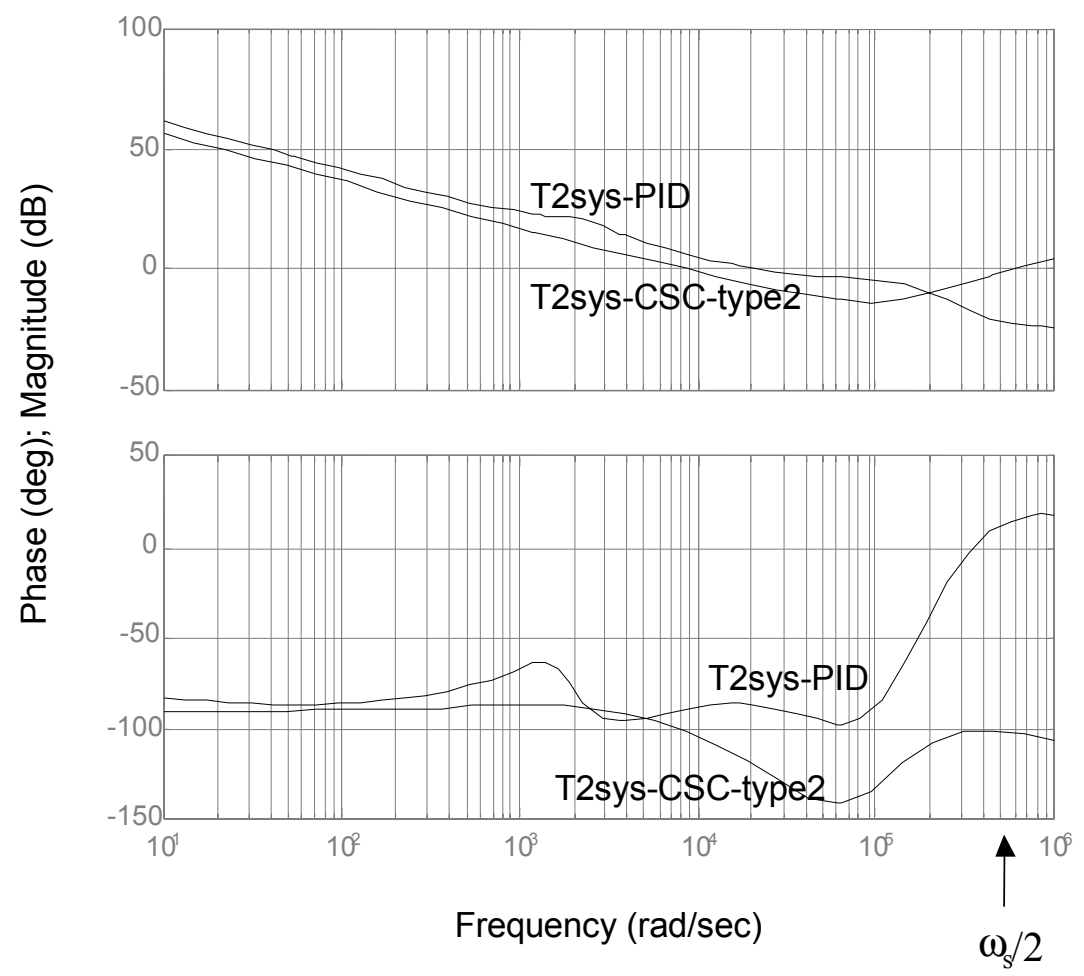

Figure 6.2-1 - Small signal loop gains for the classical design and an improved PID design

The BW for the system using the classical, type 2, CSC is approximately $1.6 \mathrm{KHz}$, with a PM of $80^{\circ}$ and infinite gain margin; The PID design has a BW of $4.7 \mathrm{KHz}$, with a PM of $100^{\circ}$ and also infinite GM. A better dynamic performance for the PID controller can be inferred, as it will be shown in the large signal, time domain, simulated results.

Comparative 25\% and 50\% nominal load step responses are given in Figure 6.2-2-(a) for the classical OpAmp CSC, Figure 6.2-2-(b) for the PID design and Figure 6.2-2-(c) for the fuzzy controller with relatively low gains, 40 for the Master (converter \#1) and Slave \#1 (converter \#2) pair and respectively 20 for the Master and Slave \#2 (converter \#3) pair. The ranges for the error and its derivative are [-1 1$]$ and respectively [-10 10$]$, following the design methodology given in the second half of paragraph 5.1. The FLC has the structure in Figure 5.13 with the membership functions layout in Figure 5.1-4, but with different signs and gains accordingly with the system connections for this case; there is no conceptual difference in the design, only its parameters are tuned for the specific application in this simulation example. 
(a)

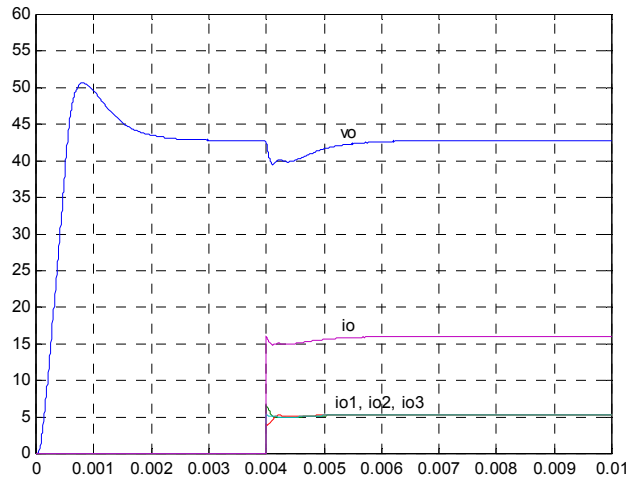

(b)

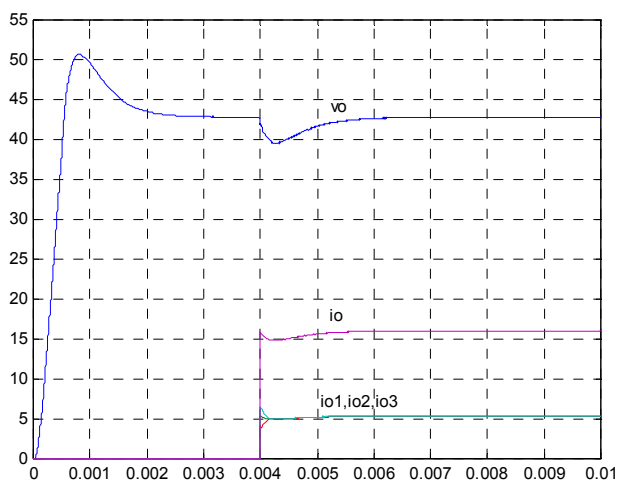

(c)

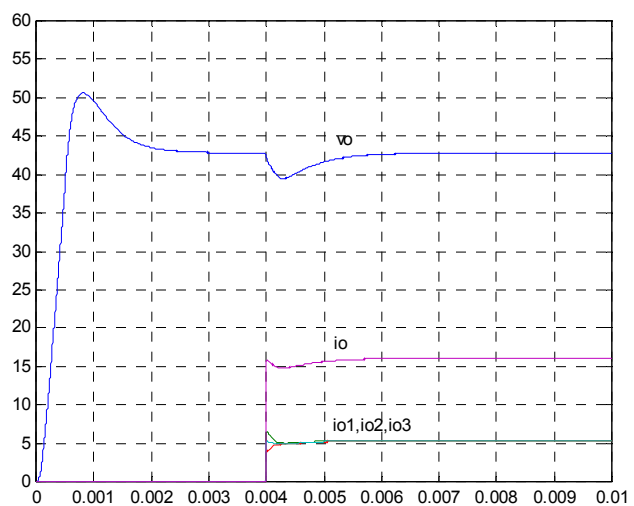

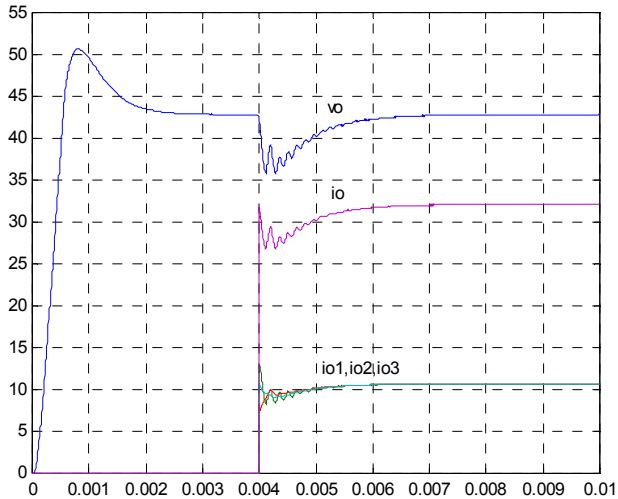
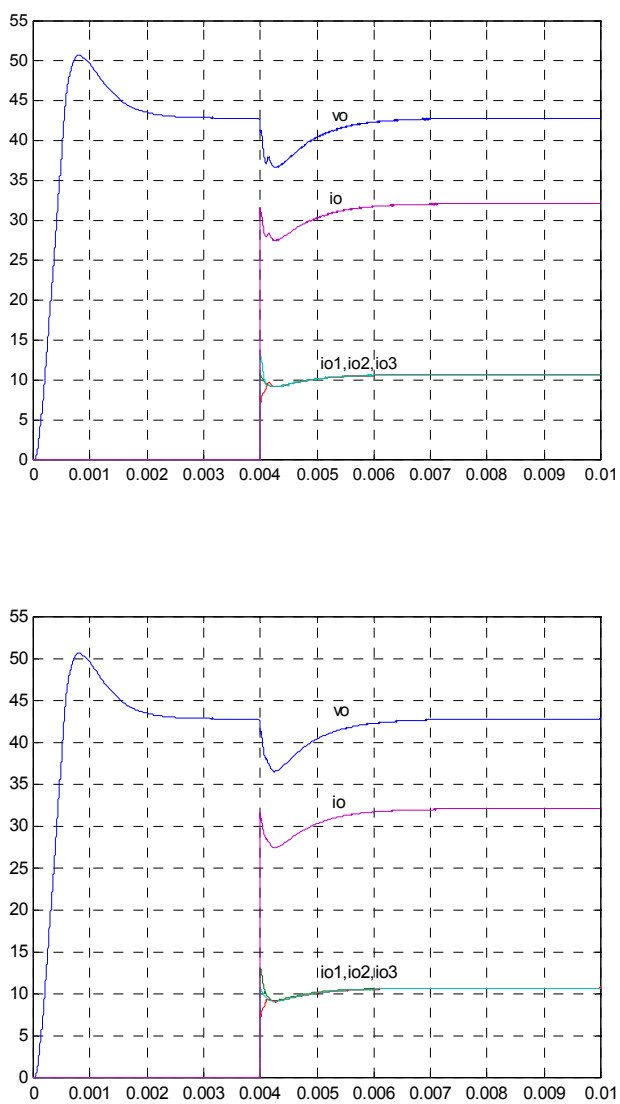

Figure 6.2-2 - Step load responses for 25\% and 50\% of nominal load: (a)-System with the classical CSC in Figure 5.1-2; (b)-System employing a PID CSC; (c)-System using a Fuzzy Logic CSC

Figure $6.2-3$ shows the comparative $25 \%$ and $75 \%$ nominal load step responses for the same cases in Figure 6.2-2 above. 
(a)

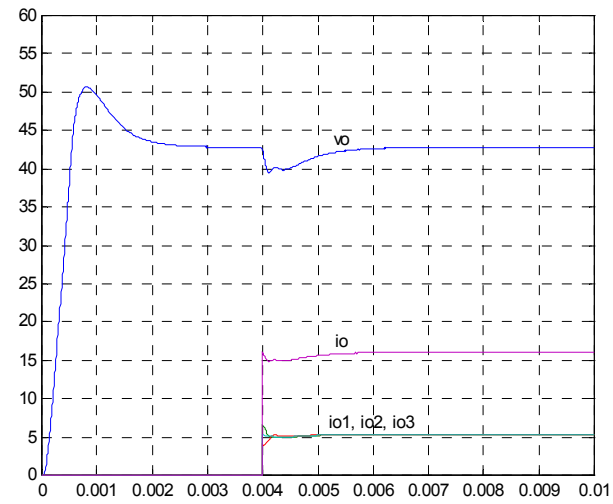

(b)

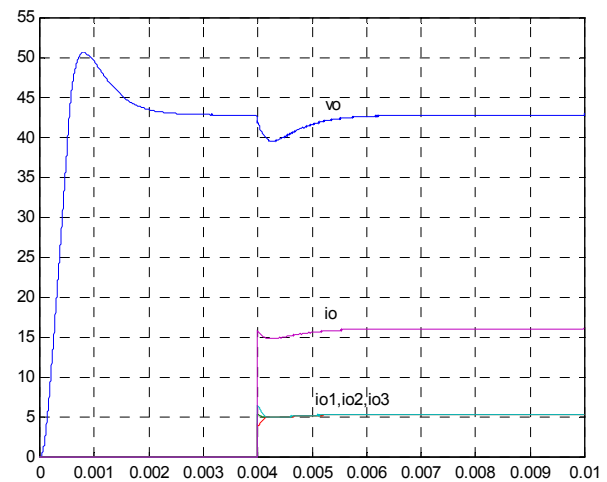

(c)

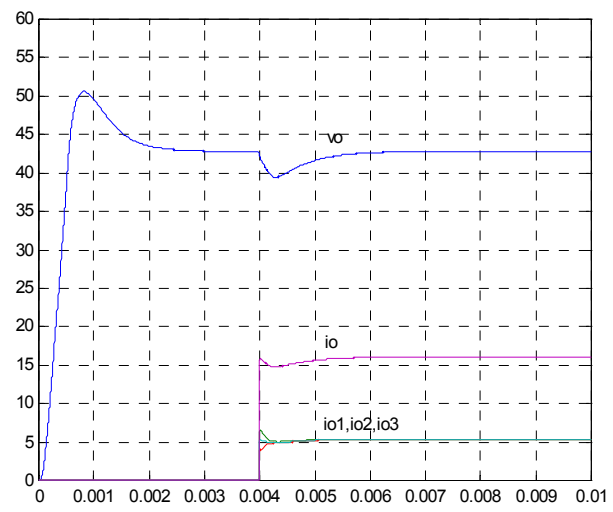

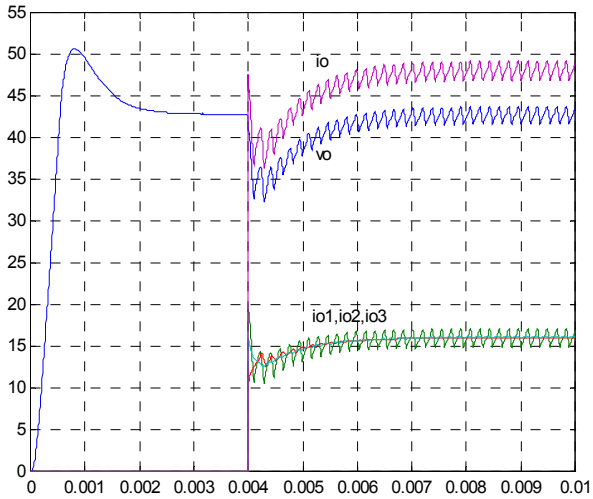
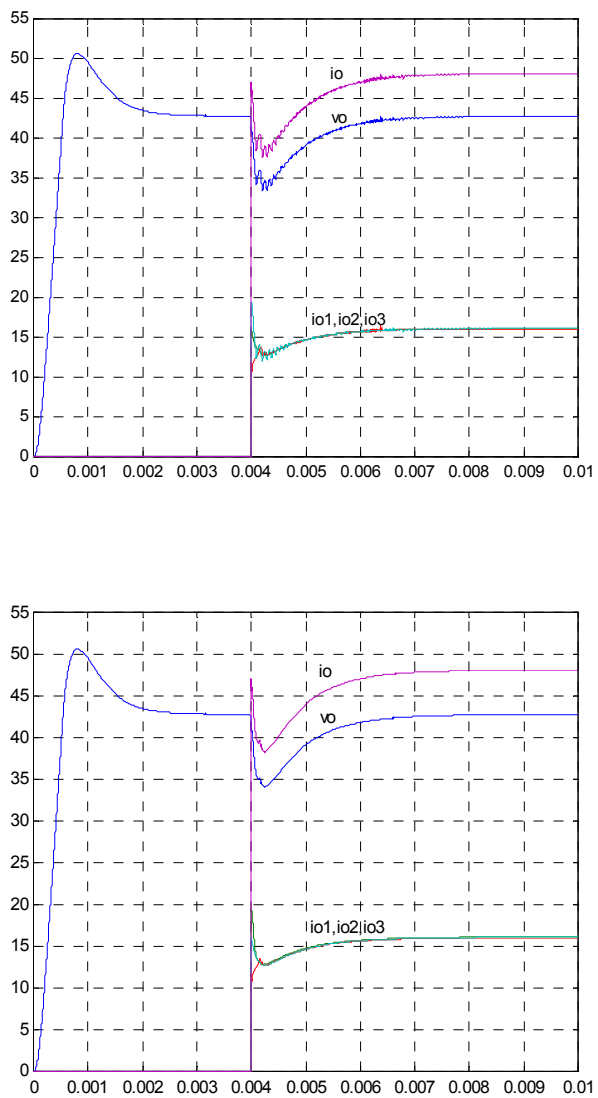

Figure 6.2-3 - Step load responses for $25 \%$ and $75 \%$ of nominal load: (a)-System with the classical CSC in Figure 5.1-2; (b)-System employing a PID CSC; (c)-System using a Fuzzy Logic CSC

As noted in the first example, in paragraph 5.1.1 a substantial improvement in the currents overshoot/settling time as well as the overall sharing is noted. Almost identical responses for the three step load cases when using the fuzzy control approach shows the validity of fuzzy control in dealing naturally with the nonlinear character of the system. 
The low performance index in the traditional cases is due to the fact that a conventional linear controller, designed only on a small signal model, is valid only near the operating point and not for the entire plant trajectory. The fuzzy approach gives also a simple practical way of overcoming modeling complexity by using heuristic judgment. Figure $6.2-4$ depicts the CSC control effort plots for the same cases shown in Figure 6.2-3.

(a)
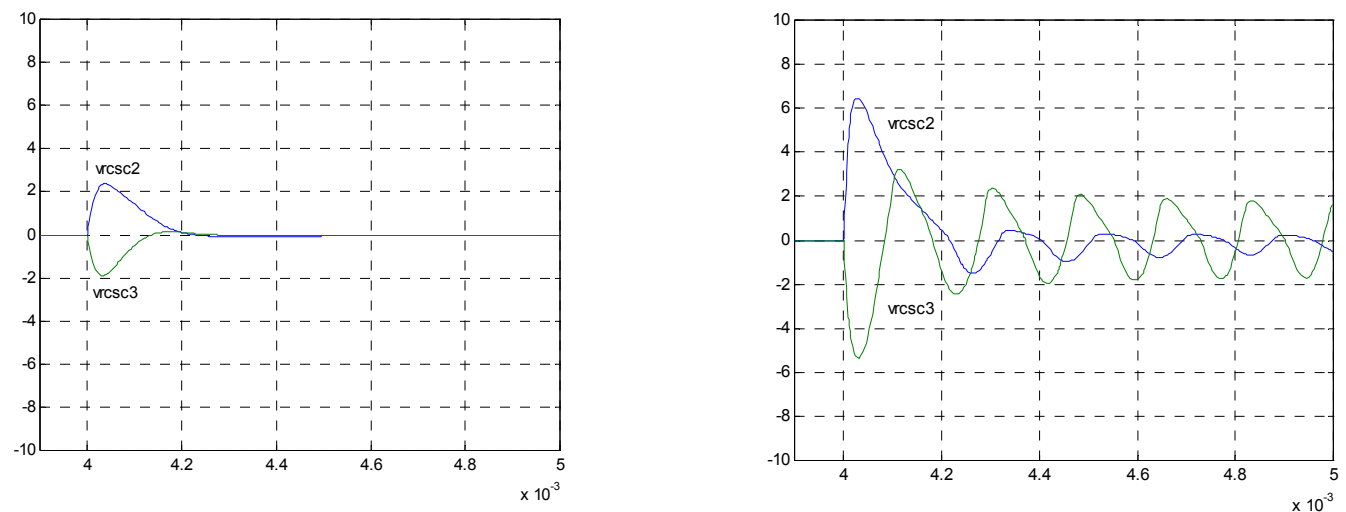

(b)
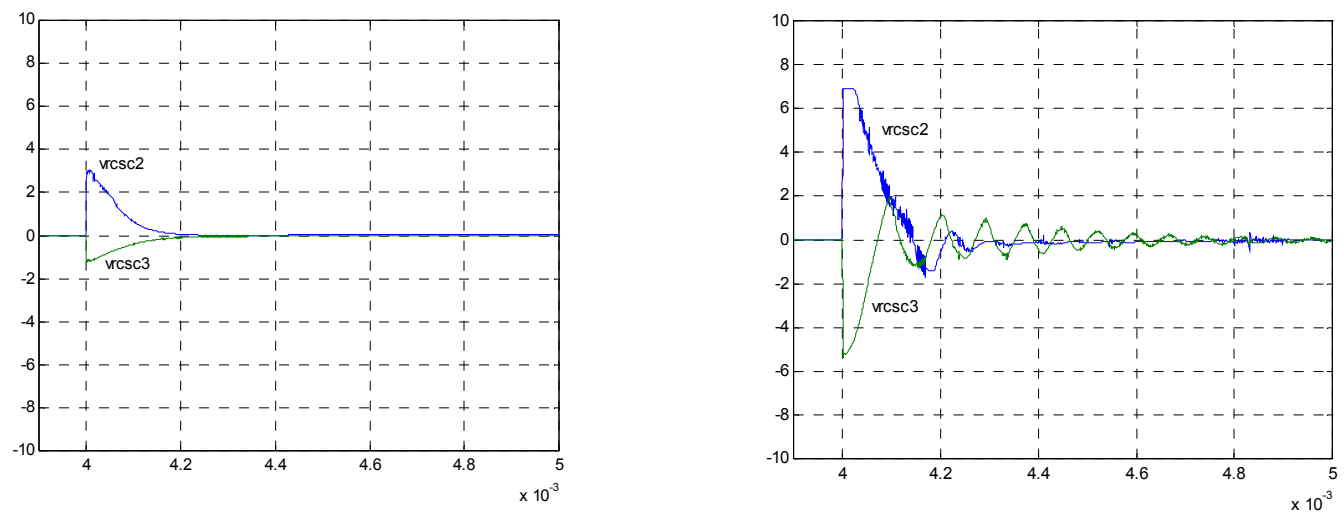

(c)
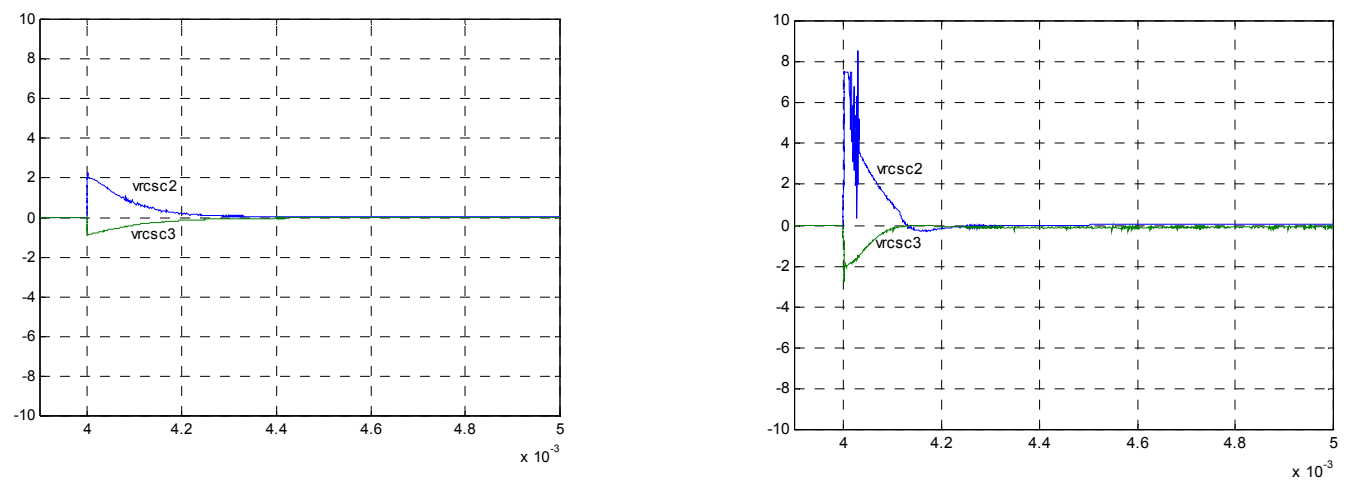

Figure 6.2-4 - CSC Control Effort for 25\% and 75\% load steps: (a)-System with the classical CSC; (b)-System employing a PID CSC; (c)-System using a Fuzzy Logic CSC 
One can again see that the fuzzy controller gives the minimal needed effort to control the system current sharing. The use of the compensator in Figure 5.1-2, is the slowest and requires the most control effort, while the PID controller gives an intermediary performance. The noiselike areas of the waveform are given mainly by numerical errors in Matlab. Cleaner waveforms can be obtained by increasing the precision of the numerical methods and the resolution with which the graphs are printed, however the simulation time becomes very long and impractical.

To ensure stability, the Lyapunov descent path supervisory controllers in Eqs. (4.5-8) (4.5-10) were used in an oscillating system with higher gains for the fuzzy compensator, which is illustrated in Figure 6.2-5 below.

(a)

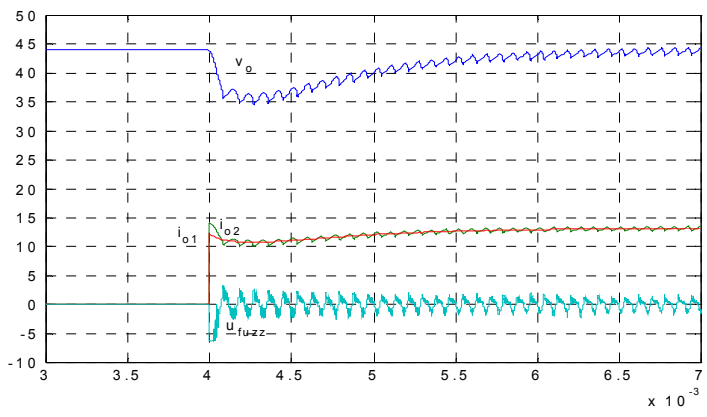

(b)

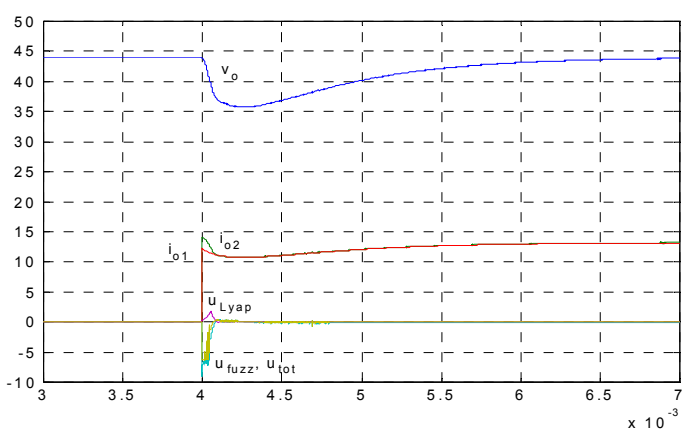

(c)

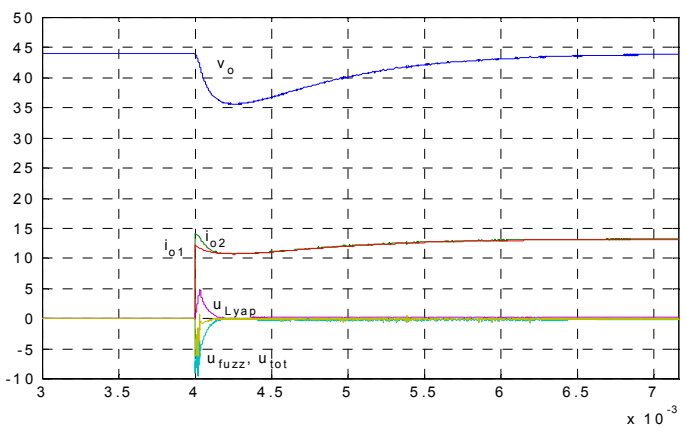

Figure 6.2-5 - Lyapunov stability adaptation waveforms: (a)-Stand alone Fuzzy Logic Controller;

(b)-Combined FLC and Lyapunov supervisory controller (78), $K_{I}=0.05, K_{2}=2$;

(c)-Combined FLC and Lyapunov supervisory controller (79), $K_{I}=0.05, K_{2}=0.5$. 
Two converters sharing a 44A load, were used in a $60 \%$ step load case simulation with an oscillatory behavior given by a high gain fuzzy controller, i.e., 50, Figure 6.2-5-(a). The control laws (4.4-8) - Figure 6.2-5 (b) and (4.4-9) - Figure 6.2-5-(c), were appended continuously in a summed, supervisory controller, (4.4-10). It can be seen that the Lyapunov supervisory controller has an immediate regulatory action and brings the error to zero. This is done by keeping the total gain from exceeding the value that would bring the system to oscillate, through the algebraic sum of the two controllers On an off-line gain adjustments are possible, based on each practical application.

The drawbacks of the fuzzy control could be a lower noise immunity together with different errors, e.g. quantization errors, associated with any digital controller, for example. This can be addressed on an application specific basis either through conventional, analog or digital filtering, or by building filtering capabilities in the fuzzy controller itself, e.g. by using triangular or Gaussian fuzzifiers instead of the singleton fuzzifiers, [36]. Quantization and other types of errors are present in any practical implementation but more and more precise and high speed processor are becoming available to the practical engineer. The main objective of this study is to introduce the new fuzzy logic control application, develop the associated mathematical theory and prove the concept and its advantages through comparative simulation verification with the existing, classical, methods.

As mentioned at the end of Chapter 4, an additional stabilizable controller will only be needed if a very high gain fuzzy controller is desired. This is because, due to the internal structure of the fuzzy logic compensator described in Chapter 2, when the error is at maximum, the control output will saturate Negative Big or Positive Big and if we do not use a very high gain, we would have the system's response to a constant command on the DC-DC module reference input; this may be slower but it is stable in almost all cases. 


\section{Chapter 7}

\section{THESIS CONTRIBUTIONS AND SUGGESTIONS FOR FUTURE RESEARCH}

This final chapter summarizes the main results and contributions of the thesis in the order they were presented in the previous chapters, together with outlining several directions for future research.

\subsection{Thesis Contributions}

\section{Fuzzy Logic as a New Control Approach for Paralleled DC/DC Converters Current}

Sharing Control: The primary subject and contribution of the thesis is to introduce, analyze and design fuzzy logic controllers for the current sharing compensator of master-slave DC/DC paralleled converter systems. This was introduced for the first time for this type of applications as part of the same study, [2]. Even though explored in the past for individual modules, no investigation had yet been done for applying the same concept to the more complicated parallel system; the work here offers the novelty of a first study in this direction. Individual converter modules are well established in the industry in terms of control implementation and size, where a complex digital controller does not offer a viable alternative. However, in the case of large, distributed systems, due to their complex, nonlinear behavior, especially for large load and line variations, modern digital controllers are fully justified and needed during the next generation of improved DC/DC power conversion and management.

The implementation offers a significantly improved performance over solidly developed classical OpAmp designs. One approach is to first introduce an improved PID, Eq. (5.1-2), as an upgrade for the existing "Type 2" OpAmp compensators and then, to employ the PID as an expert system in partially developing the rules for the Fuzzy Logic Controller, Fig. 5.1-3 and Fig. 5.1-4. This offers a method of calculating the singleton specific values of the consequents, while the other part of the design comes from the knowledge of the control action on the plant itself. In this respect, the design falls mostly in the category of Direct Adaptive Fuzzy Controllers, even 
though it could also be approached, for more complicated designs, as a combination of Direct and Indirect Adaptive Fuzzy Controllers, [36].

A three-range partition, negative $(\mathrm{N})$, zero $(\mathrm{Z})$ and positive $(\mathrm{P})$, can be chosen for the inputs, $e$ and $\dot{e}$. A more refined five-range partition, negative-big (NB), negative-small (NS), zero (Z), positive-small (PS) and positive-big (PB) is used for the output and could be also used of the input, but the results obtained so far are use the simpler, three range input partition. Based on the usual action of a PD cell, by looking at its output range as a function of the input range, a rule-based inference is chosen for each consequent using a simplified "ith height" rule corresponding to consequent $\# i$. Further optimization in partitioning the input spaces, as well as using neuro-fuzzy techniques in an adaptive grid of controllers, [24]-[25], can be considered.

A substantial improvement in the transient performance and control effort is noted in the case of the PID and especially of the fuzzy logic controller, Fig. 5.1-5 - Fig. 5.1-7 and Fig. 6.2-1 - Fig. 6.2-3. The simulation results show a very good parameter insensitive transient response in load step responses from $25 \%$ to $75 \%$ of the nominal load. The technique benefits also from the heuristic approach to the problem that overcomes the complexity in modeling such systems and, hence, offers a practical engineering tool, amenable to both analog and digital implementations. Almost identical responses for widely different step load cases, when using the FLC, shows its validity in dealing naturally with the nonlinear character of the system, due mainly to its "universal approximator" feature, which is not the case for a conventional linear controller, designed on a small-signal model that is valid only near the respective operating point.

Lyapunov-based Design for the Fuzzy Logic Current Sharing Control: The stability problem was tackled in Chapter 4 by introducing, for the first time, a Lyapunov analysis of the closed loop DC/DC converter, Eqs. (4.4-2), (4.4-6) and (4.4-8) and of the paralleled system, Eqs. (4.5-2) and (4.5-4), which uses the reference voltage as the control input for the current sharing loop, as is the case in the industry; this is another contribution of the thesis. Even though several schemes were considered for the individual modules, they did not offer practicality due to the contradictory trends of miniaturization for the converter and computational complexity for the controllers. Since for distributed systems the volume constraints are less stringent than for a single module converter, the complexity of such systems fully justifies the advanced control algorithms, whereas individual modules can successfully be compensated with traditional 
approaches. In Section 5.2 is shown a general proof of existence for an adaptive Lyapunov trajectory if a fuzzy controller of the type used here is employed, [36], provided that boundedness can be achieved for the system's salient parameters. This algorithm is very general and it does not offer a direct implementation since a high degree of trial and error gain adjustments is needed in order to see any results, for each application. However, a Lyapunov supervisory control was successfully implemented considering the specific Lyapunov function developed in Chapter 4, for two parallel DC/DC converter modules, Eqs. (4.5-8) and (4.5-9).

Based on all the previously introduced results, Chapters 5 and 6 applied the new idea of using fuzzy logic for improved large signal performance for the current sharing loop and, respectively, showed comparative, improved, simulated results with classical compensator designs.

\section{Unification of DC/DC Converters Averaged Models with Modern Control Analysis and Design (Robustness, Disturbance Rejection and Tracking Highlights): In order to} gain insight into the system's real structure so that the new fuzzy logic controller can be developed, the existing results on averaged circuit models for switching power converters have been extended in a modern control theory formalism. Chapter 2 integrates those models in a disturbance rejection and robustness framework, Eqs. (2.2-20), (2.2-21) and (2.3-3). Then, using the unterminated model, [17] - [31], in this new formalism clarifies how the plant structure changes under a load disturbance and offers the possibility of a straightforward, modern control system modeling, Eqs. (2.5-3), (2.5-15) and (2.5-25). If the load terminated, full, model is considered, it was shown that a load change actually alters the plant structure by altering the terms inside the state space model matrices; the existing small signal model considers the line and load variations only as small signal parameters of the associated Bode plots. For an ideal plant model, this type of structure can be easier highlighted and the load impedance variation can be thus factored out. Equations (2.3-2), (2.3-3) and (2.4-4) quantify its contribution for resistive and respectively constant power loads. In this way mathematical simulators, such as Matlab/Simulink, can be employed for describing and developing converter systems in a way that allows new, emerging control techniques to be used. Using Spice-based circuit simulators alone, even though it offers a high power circuit check-up tool, is most times insufficient for developing a contribution in a highly interconnected, nonlinear system. 
The new converter model block diagram was thus derived and is given in Fig. 2.5-2. Regarding the PCMC control strategy, the thesis also clarified, for the first time, that for large signal paralleled DC-DC systems there is no real advantage in separating the converter circuit at the modulator output in order to use the modulator's gain expression that comes out from the signal theory for that circuit. The integrated box level results are practically identical if the mathematical averaged model is used as a whole, for the overall converter module. That is how all the models start, but they are then split and subsequently modified at the PWM modulator output. Reference [7] comes the closest to addressing this issue, highlighting both forms, but with a different goal, i.e. trying to include the effects of " $H_{e}(s)$ " in a state space form. Those forms are useful in the single converter small signal, Bode analysis, but they would just hide the real control issues revealed here, when used for the paralleled system analysis.

Chapter 3 continued with detailing the Master-Slave paralleled system and laid the foundation for current sharing fuzzy logic control by showing that the control problem in this case is a tracking problem where the current sharing error is directly related to the error in the outputs, Fig. 3.1-2, Fig. 3.1-3 and Eqs. (3.1-5) and (3.1-6). It was shown that if the detailed, nonlinear, model of the converters, including parasitics, is employed, a very complicated equation, (3.1-7), for the state error, is obtained. This equation is therefore not useful for a closed-form control law implementation. Hence, the analysis and design of the CSC was done employing the simplified, two identical converters model, (3.1-9), which offers a clearer analytical form, while the validity and fine tuning of the design was done using the complete model, which considers all the parameter variations among the converters. This further justifies the use of the fuzzy logic approach.

\section{Extension of the Small Signal Model Block Diagram to Include the Reference Voltage}

Term: As mentioned in Chapter 2, for an individual regulator module the terms in $v_{\text {ref }}$ contribute nothing to the dynamics, and all present small signal models derive their salient transfer functions based on this assumption. The study here introduced and calculated the reference-tooutput transfer function by considering the voltage reference term, since it is the control input for the paralleled system current sharing, which adjusts each slave converter output so its current tracks that of the master. The Reference-to-Output transfer function follows the structure of the outer loop gain, $T_{2}$, but includes a proportional control component term due to the feed-through 
action of the OpAmp, from its non-inverting input, where $r$ is applied. Details are shown in the modeling done in Paragraph 2.5.1, Fig. 2.5-3 and Eq. (2.5-32).

\subsection{Suggestions for Future Research}

There are several questions that are addressed, but not entirely answered in this thesis. Some of them arise in the light of the results that are obtained. The subsequent paragraphs outline some of these as possibilities for future research.

Line Disturbances: The converter model equations include the line perturbation, $v_{i n}$, which, unlike the load disturbance, comes in explicitly, as an exogenous source in the respective models, and hence it is easier to sense and handle. In addition, the significant factors containing $v_{i n}$, are purely linear. This research concentrated on the load side, since it is the main control problem in the paralleled system current sharing control. Having a good response to large line variations would alleviate the EMI and general noise coupled both in each converter and outside the system. The problem is simply of minimizing the effects of an external disturbance entering the system linearly. In this respect, there are already good small signal control approaches, using a "feedforward" term, [39], [41]. A future research path could try to include the full, large signal model, in which $v_{i n}$ enters in also through some smaller bilinear terms, both through traditional and fuzzy logic control methods.

Noise Effects: $\quad$ One of the concerns with fuzzy control, as a high bandwidth equivalent controller, is noise immunity. This can be handled on an application specific basis either through conventional, analog or digital, filtering or by building filtering capabilities in the fuzzy controller itself by using triangular or Gaussian fuzzifiers instead of the singleton fuzzifiers used for consequents. Reference [36] highlights this aspect, offering a brief, comparative performance evaluation of the different types of fuzzifier functions. A future research path would be to examine, in detail, the need and feasibility of different filtering methods when a FLC is employed. 
Constant Power Load Applications: $\quad$ Several studies have been carried out to deal with the potential instability introduced by the negative resistance equivalent with the constant power load, most of them with practical results. Depending on how large the CPL perturbation is, the system may still be stable provided there is enough damping (positive resistance) at the respective interface for that DC operating point. In those cases, using the unterminated model and applying the Nyquist criterion, one can still have a stable system, even though RHP poles exist, [31]. Each example is analyzed on an application specific basis and several means of compensating the current sharing loop have been used. The studies in [32], [33], specify the output impedance and vary the DC gain of a simple PD controller in order to "schedule" the gain for each operating point. In other cases the stability task is carried out just by reducing the bandwidth of the classical PI OpAmp controllers and making sure that no large negative resistance CPLs occur. Another successful scheme is to feed back the information about the load current level and then employ the calculation of the output and input powers in order to make the DC gain of the control-to-output function practically invariant with the load [17]. This approach offers a good performance and a more stable large signal response in the case of a CPL, since it practically zeroes out the output impedance of that converter. As a future research path, the present fuzzy logic approach could be explored in the framework of the above mentioned, existing, strategies. The new control law would have to be appended with additional constraints so that the negative resistance behavior is compensated within the feasibility limits of each application. This would be the subject of another thesis.

Full State Feedback Control: One of the features that complicated the system structure, in developing the models used previously, is that the presently established control schemes for the DC-DC converters in the industry are not a full (linear) state feedback, per se. They are a summation of different loops for each state variable, with different gains and/or OpAmp compensators in each loop. This makes the models a cumbersome form for direct modern control implementations, especially in parallel, distributed systems. One direction would be to have a linear, stable, full-state feedback for each module, which would then make the plants more amenable to be integrated in a paralleled system where the same control strategy would be continued. Another approach is to recognize the variable structure features of the DC-DC topologies and to employ a sliding mode controller, [13], [19], [36], in each module. Associated 
to that, or independently, control laws through input-output linearization and coordinate transformations, [13], [20], can also be tried. All these suggestions could be a new study path.

Practical Implementation: Based on extensive experience, the simulated results give a high degree of confidence that the proposed fuzzy logic control methods will work successfully in practice. However, it will be of interest in a future research path, to implement in hardware some of the control schemes that were proposed. The experimental work will undoubtedly generate new research directions as well as exposing some limitations.

As part of any practical design, one should consider also the effects of highly unmatched parameters among the different converters. This can include the case when different power levels and topology designs are employed and analyze not only the nominal case but include also the situation when one or several modules would fail or go into current limiting, for example.

In trying to come up with a control strategy that would easily be implemented, one can employ part of the classic control dedicated ICs in the industry, such as the TI/Unitrode 3907, Max. Current Master-Slave Current Sharing Controller, to use its current sharing error output as the input to a digital, or analog, CSC fuzzy logic section, for the rest of the processing. For this part, a DSP setup or, better yet, an embedded micro-controller supporting fuzzy logic instructions and also being faster, can be used. Regarding the speed aspect, since DC/DC converters are high bandwidth (fast) plants, a high-speed analog fuzzy logic implementation may turn out to be very successful since triangular membership functions should be fairly easy to realize using OpAmps. A successful fuzzy-logic-control-employed embedded controller is the Motorola MC68HC12 chip, which is also available as part of a first stage development, evaluation board, 68EVB912B32UM/D. Several companies, such as IMAGEcraft and PEMicro, offer hardware (PC interfaces, RAM) and software interfaces. IMAGEcraft, offers a convenient integrated C-compiler and development environment, HC12. The new Matlab interfaces for code generation and interfacing, such as Real Time Workshop and XPCTarget, as well as its Hardware in the Loop and PC in the Loop new products, may be explored. Of course, many other hardware resources, both analog and digital, can be explored; they can all be considered as part of another thesis future research, for this type of control interface. 


\section{REFERENCES}

[1] J.D. van Wyk, F.C. Lee, "Power Electronics Technology at the Dawn of the new Millennium-Status and Future”, Proc. of IEEE PESC'99, Vol. 1, pp. 3-13.

[2] B. Tomescu and H.F. VanLandingham, "Improved Large Signal Performance of Paralleled DC/DC Converters Current Sharing Using Fuzzy Logic Control”, IEEE Trans. PE, vol. 14 No. 3, May 1999, pp. 573-577.

[3] R.D. Middelbrook and Slobodan Cuk, " A general unified approach to modeling switching converter power stages", Advances in Switched Mode Power Conversion, Tesla Co. vol. 1, pp. 73-89.

[4] V. Vorperian, "Simplified Analysis of PWM Converters Using the Model of the PWM Switch, Part I: Continuous Conduction Mode", Part II: Discontinuous Conduction Mode", VPEC Seminar, Sept. 1989.

[5] Jian Sun, "Unified Averaged Switch Models for Stability Analysis of Large Distributed Power Systems", IEEE APEC Proc., New Orleans, LA, 2000.

[6] R. Ridley, "A new, Continuous-Time Model for Current-Mode Control”, IEEE Trans. PE, Vol. 6 No. 2, April 1991.

[7] R. Tymerski, D. Li, "State-Space Models for Current Programmed PulsewidthModulated Converters", IEEE Trans. PE, Vol. 8, No. 3, July 1993, pp. 271 - 278.

[8] A.R. Brown and R. D. Middlebrook, "Sampled-Data Modeling of Switching Regulators", IEEE PESC'81 Proceedings, pp. 349-369.

[9] R. Ridley, B. H. Cho and F.C. Lee, "Analysis and Interpretation of Loop Gains of Multiloop-Controlled Switching Regulators”, IEEE Trans. PE, vol. 3, No. 4, Oct. 1988.

[10] R. W. Erickson, S. Cuk and R.D. Middlebrook, "Large-signal modeling and analysis of switching regulators", IEEE PESC Rec., 1982, pp. 240 - 250.

[11] K. Harada and T. Nabeshima, " Large Signal Transient Response of a Switching Regulator”, IEEE PESC Rec., 1981, pp. 388 - 394.

[12] G. Salut, J.C. Marpinard and M. Valentin, "Large signal feedback control for power switching conversion”, IEEE PESC Rec., 1985, pp. 741 - 750. 
[13] S. R. Sanders and G. C. Verghese, "Lyapunov-Based Control for Switched Power Converters”, IEEE Trans. PE vol. 7 No. 1, January 1992.

[14] F. Chen and X. S. Cai, " Design of feedback control laws for switching regulators based on the bilinear large model”, IEEE PESC Rec., 1989, pp. 468 - 475.

[15] I. Derese and E. Noldus, "Design of Linear Feedback Laws for Bilinear Systems", INT. J. Control, Vol. 31, No. 2, pp. 219-237.

[16] L. Malesani, L. Rossetto, G. Spiazzi, P. Tenti, "Performance Optimization of Cuk Converters by Sliding-Mode Control”, Proc. APEC 1992, pp. 395 - 402.

[17] S. Hiti and D. Borojevic, "Robust Nonlinear Control for Boost Converter", IEEE Trans. PE, vol. 10, No.6, November 1995, pp.651-658.

[18] B. Tomescu and H.F. VanLandingham, "Disturbance Rejection and Robustness Considerations in DC/DC Converters", IEEE PESC Proc., Charleston, SC, June 1999, pp. $1204-1210$.

[19] H. Sira-Ramirez, "Sliding Motions in Bilinear Switched Networks", IEEE Trans. CAS, Vol. 34, No. 8, August 1987, pp. 919-933.

[20] H. Sira-Ramirez, "Exact linearization in Switched Mode DC to DC Power Converters", Int. Journal of Control, Vol. 50, No. 2, 1989, pp. 511-524.

[21] P. Mattavelli, L. Rossetto, G. Spiazzi, P. Tenti, "General - Purpose Fuzzy Controller for DC/DC Converters”, IEEE PESC 1995 Record, pp. 723 - 730.

[22] F. Ueno, and al., "Regulation of Cuk Converters using fuzzy Controllers", Proc. Intelec'91, pp. 261 - 267.

[23] W.-C. So, C.K. Tse and Y.-S. Lee, "Development of a Fuzzy Logic Controller for DC/DC Converters: Design, Computer Simulation and Experimental Evaluation", IEEE Trans. PE, vol. 11,No.1, January 1996, pp. 24 - 32.

[24] B. Tomescu and H.F. VanLandingham, "Neuro-Fuzzy Multi-Model Control using Sugeno inference and Kohonen tuning in parameter space", Proc. of IEEE Conf. on Man, Syst. and Cybernetics, Orlando, FL, 1997.

[25] J. Rajagopolan, Y. Panov, B. Tomescu and Y. Guo, "Stability assessment of distributed power systems used in ISSA", NASA LeRC quarterly report, VA Polytech. Inst. \& SU VPEC, Dec. 1995.

[26] J. Rajagopolan, K. Xing, Y. Guo and F.C. Lee, "Modeling and Dynamic Analysis of Paralleled DC/DC Converters with Master/Slave Current Sharing Control", Proc. APEC'96, pp. 678-684. 
[27] Y. Panov, J. Rajagopolan and F.C. Lee, "Analysis and Design of N Paralleled DC-DC Converters with Master-Slave Current Sharing Control”, Proc. of APEC 97, pp. 436-442.

[28] V.J. Thottuvelil and G. Verghese, "Analysis and Control Design of Paralleled DC-DC converters with current sharing", Proc. of APEC 97, pp. 638-646.

[29] K. Siri, C.Q. Lee and T.F. Wu, "Current Distribution Control for Parallel-Connected Converters", IEEE Trans. AES, vol. AES-28, No. 3, July 1992, pp. 829-850.

[30] S. Luo, Z. Ye, R.L. Lin and F.C. Lee, "A Classification and Evaluation of Paralleling Methods for Power Supply Modules”, IEEE PESC, Charleston, SC, 1999, pp. 901-908.

[31] L.R. Lewis, B.H. Cho, F.C. Lee and B.A. Carpenter, "Modeling and Analysis of Distributed Power Systems", Modeling, Analysis and Design of PWM Converters, Vol.2, VPEC Publication Series, pp. $505-514$.

[32] X. Feng., Z. Ye, Kun Xing, F.C Lee, D. Boroyevich, "Impedance Specification and Impedance Improvement for DC Distributed Power Systems", IEEE Proc. PESC'99, Vol. 1, pp. 889 - 900, Charleston, SC.

[33] Z. Ye, D. Boroyevich, Kun Xing and F.C. Lee, "Design of Parallel Sources in Distributed Power Systems by Using Gain-Scheduling Techniques”, IEEE Proc. PESC'99, Vol. 1, pp. $161-165$, Charleston, SC.

[34] M. Scheffer, A. Bellini, R. Rovatti, A. Zafarana, C. Diazzi, “A Fuzzy Analog Controller for High Performance Microprocessor Power Supply”, Proc. EUFIT’96, Germany.

[35] M. Jordan, "Load Share IC Simplifies Power Supply Design”, Proc. of HFPC'91, pp. 65-76.

[36] Li-Xin Wang, “A course in fuzzy systems and control”, Prentice Hall, 1997.

[37] A. Isidori, "Nonlinear Control”, Springer Verlag, 1992.

[38] H. Khalil, "Nonlinear Control”, Macmillan Publishing”, New York, 1992

[39] D. Mitchell, "DC-DC Switching Regulator Analysis", McGraw-Hill, 1988.

[40] R. P. Severns and G. E. Bloom, "Modern DC-to-DC Switchmode Power Converter Circuits", Van Nostrand Reinhold, 1985.

[41] A. Kislovski, R. Redl and N. Sokal, "Dynamic analysis of Switching-mode DC/DC Converters", Van Nostrand Reinhold, 1991. 
[42] J.S.R. Jang, Ned Gulley, "Fuzzy Logic Toolbox for Use with Matlab", The MathWorks Inc. Printing, 1995.

[43] J. S. Bay, "Fundamentals of Linear State Space Systems”, WCB/McGraw-Hill, 1999.

[44] W. L. Brogan, "Modern Control Theory”, Prentice Hall, 1991. 


\section{Appendix A}

\section{FUZZY LOGIC CONTROL FUNDAMENTALS}

Fuzzy theory was initiated by Lotfi Zadeh in 1965 who had also worked in developing the concept of state, which is at the basis of modern control theory, [36]. As the control methods evolved in great detail, he thought that classical control theory had put too much emphasis on precision and therefore could not handle complex systems in a very practical manner. The new trend generated many debates and even though there are still ongoing discussions, it proves to be an excellent way of handling complicated, nonlinear systems.

\section{A.1 Fuzzy Sets and Membership Functions}

Let $U$ be the input space, or universal set, which contains all the possible elements of concern in each particular context or application. This input space is many times referred to as the Universe of Discourse, somehow a peculiar name. Contrary to a classical or crisp set, a fuzzy set is a set in the universe of discourse without a crisp, clearly defined boundary. It can contain elements with only a partial degree of membership (membership method). A classical set is a container that wholly includes or excludes any given element. Another way of defining a classical set, in opposition to a fuzzy one, is by listing all of its members (the list method) or by specifying the properties that must be satisfied by the members of the set (rule method), [36], [42].

Example: 1. Consider the set of tall people. If the set of tall people is given the well defined, crisp boundary of a classical set, we might say that all people taller than six feet are officially considered tall. But, such a distinction is not fully realistic. If one would however consider a smooth curve from "not-tall" to "tall", then the transition would make more sense. Both people are tall to some degree but one is significantly less than the other. The output axis 
would be a number between 0 and 1, known as the membership function, which will be introduced next; for this example, it would describe the "degree of height" for tall people.

2. Linked to the control problem addressed in this thesis, consider $U$ as the set of all the control input values of a Current Sharing Compensator (CSC). Then, one fuzzy set, denoted $A$, can be "All the control input values that are negative big".

A membership function (MF) is a function that defines how each point in the input space is mapped to a membership value, or degree of membership, between 0 and 1 . The following definition for a fuzzy set can thus be given:

Definition A.1-1: A fuzzy set $A$ in the universe of discourse $U$ is a set that is characterized by a membership function $\mu_{A}$ that takes values in the interval [ 011$]$,

$$
A=\left\{x, \mu_{A}(x) \mid x \in U\right\}
$$

In contrast, if one were to define the membership function of a classical set, it would be a function that can only take two values, zero and one. It can be seen that by characterizing a fuzzy description with a MF, we actually de-fuzzify the fuzzy description.

The fuzzy set membership function itself can be an arbitrary curve shape that we can define as a function that suits us from the point of view of simplicity, convenience, speed and efficiency. The simplest, and very popular, membership functions are formed using straight lines. They are the triangular and the trapezoidal MFs, which can have different symmetries. A comparative example of a triangular membership function for Example 1 above, both for a crisp and a fuzzy set is given in Figure A-1, below. 

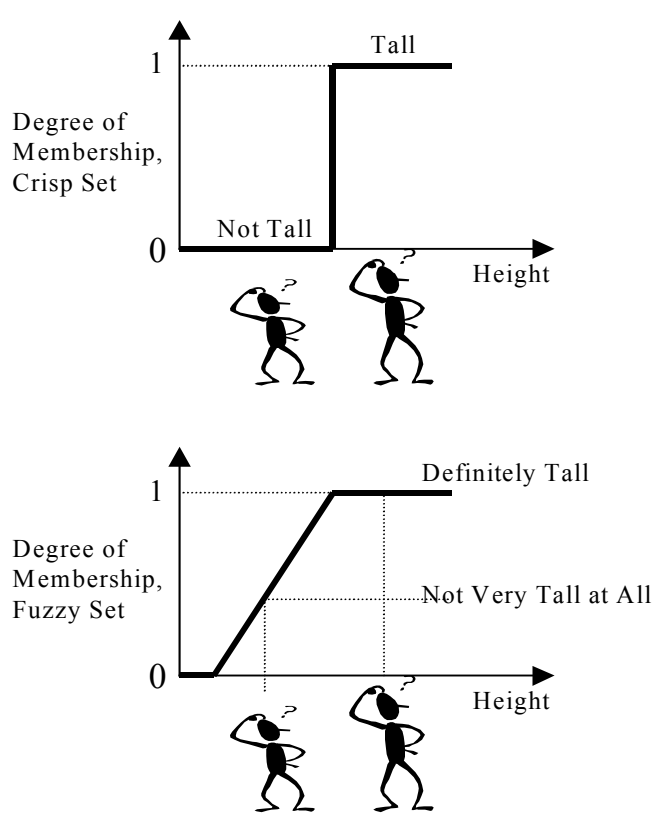

Figure A-1 - Fuzzy vs. Crisp Sets illustration

Gaussian Bell shaped MFs are also used and the parameters defining any MF shape can be used as variables for on-line or off-line training, in order to optimize our control system, for example.

\section{A.2 Basic Operations and Relations in Fuzzy Sets}

Assume that $A$ and $B$ are fuzzy sets defined in the same universe of discourse, $U$.

Definition A.2-1: The equality, containment, complement, union and intersection of two fuzzy sets, $A$ and $B$ are defined as follows.

- $A=B$ if and only if (iff) $\mu_{A}(x)=\mu_{B}(x), \forall x \in U$.

- $A \subset B$ iff $\mu_{A}(x) \leq \mu_{B}(x), \forall x \in U$.

- The complement of $A$ is a fuzzy set, $\bar{A}$, whose membership function is defined as

$$
\mu_{\bar{A}}(x)=1-\mu_{A}(x)
$$

- The union of $A$ and $B$ is a fuzzy set in $U$, denoted by $A \cup B$, whose membership function is defined as 


$$
\mu_{A \cup B}(x)=\max \left[\mu_{A}(x), \mu_{B}(x)\right] .
$$

- The intersection of $A$ and $B$ is a fuzzy set in $U$, denoted by $\mathrm{A} \cap \mathrm{B}$, whose membership function is defined as

$$
\mu_{A \cap B}(x)=\min \left[\mu_{A}(x), \mu_{B}(x)\right] .
$$

Definition (A2-1) introduces actually functions, $t:\left[\begin{array}{ll}0 & 1\end{array}\right] \times\left[\begin{array}{ll}0 & 1\end{array}\right] \rightarrow\left[\begin{array}{ll}0 & 1\end{array}\right]$ and respectively $s:\left[\begin{array}{ll}0 & 1\end{array}\right] \times\left[\begin{array}{ll}0 & 1\end{array}\right] \rightarrow\left[\begin{array}{ll}0 & 1\end{array}\right]$, that transform the membership functions of fuzzy sets $A$ and $B$ into the membership function of the intersection and respectively union of $A$ and $B$,

$$
\begin{aligned}
& t\left[\mu_{A}(x), \mu_{A}(x)\right]=\mu_{A \cap B}(x) . \\
& s\left[\mu_{A}(x), \mu_{A}(x)\right]=\mu_{A \cup B}(x) .
\end{aligned}
$$

Other functions can also be employed, but they are not within the scope of this study. It can be shown, [36], that the $s$ and $t$ functions can also be used as norms in the fuzzy MFs space, and they are referred as $t$-norm and respectively $s$-norm.

Definition A.2-2: Let $X$ and $Y$ be two arbitrary classical, crisp, sets. The Cartesian Product of $X$ and $Y$, denoted $X \times Y$, is the non-fuzzy set of all ordered pairs, $(x, y)$ such that $x \in X$ and $y \in Y$, that is,

$$
X \mathrm{x} Y=\{(x, y) \mid x \in X \text { and } y \in Y\}
$$

The definition can be expanded to a higher number of sets. A non-fuzzy relation among the non-fuzzy sets $X$ and $Y$ is a subset of the cartesian product $X \times Y$, that is

$$
Q(X, Y) \subset X \times Y
$$

For example, $Q(X, Y)$ can be a relation named "The first element is smaller that the second element". Because a relation is itself a set, all of the basic set operations can be applied to it.

Definition A.2-3: A Fuzzy Relation is a fuzzy set defined in the cartesian product of the crisp sets $X$ and $Y$. Similarly to (A-6), $Q$ in $X \times Y$ is defined as the fuzzy set

$$
Q=\left\{(x, y), \mu_{Q}(x, y) \mid(x, y) \in X \times Y\right\}
$$


where $\mu_{Q}: X \times Y \rightarrow\left[\begin{array}{ll}0 & 1\end{array}\right]$ can be any $t$-norm, e.g. (A-5). As a special case, a binary fuzzy relation is a fuzzy set defined in the cartesian product of two crisp sets. This relation is usually represented by a fuzzy relational matrix, i.e., a matrix whose elements are the membership values of the corresponding pairs belonging to the fuzzy relation.

\section{A.3 Fuzzy IF-THEN Rules}

Fuzzy IF-THEN rules operate with linguistic variables as part of fuzzy propositions. Therefore we need to first define each of the two concepts.

Definition A.3-1: If a variable can take words in the natural language as its values, it is called a Linguistic Variable, where the words are characterized by fuzzy sets defined in the universe of discourse in which the respective variable is defined.

Example: Consider the control error as a variable, $e$, that takes values in the interval $U=\left[\begin{array}{ll}-1 & 1\end{array}\right]$. We define five fuzzy sets "Negative-N", "Zero-Z" and "Positive-P", as shown in Figure A-2

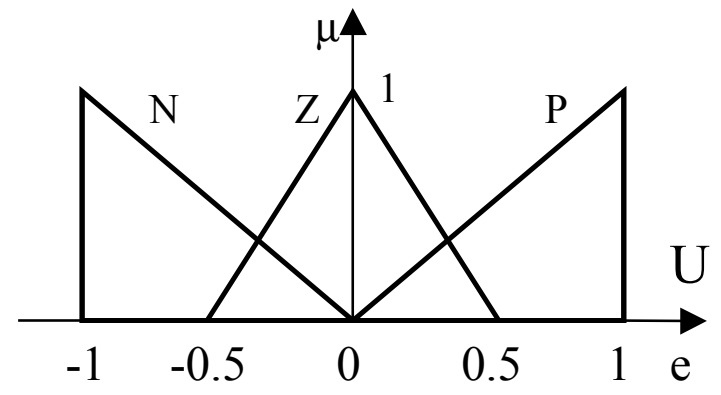

Figure A-2 - Fuzzy Membership Functions illustration

A more formal definition of a linguistic variable is usually employed, as defined by Zadeh. 
Definition A.3-2: A linguistic variable is characterized by $(X, T, U, M)$, where:

- $X$ is the name of the linguistic variable, e.g., the speed of a car or the value of a control input.

- $T$ is the set of linguistic values that $X$ can take, e.g., the control input $u \in\{$ Negative, Zero, Positive .

- $U$ is the actual physical domain in which the linguistic variable $X$ takes its quantitative, crisp, values, e.g., [0 $\left.\mathrm{U}_{\max }\right]$.

- $M$ is a semantic rule that relates each linguistic value in $T$ with a fuzzy set in $U$, e.g., $M$ would relate "Negative", "Zero" and "Positive" with the membership functions shown in Figure A-2.

- There are two types of fuzzy propositions: "atomic" and compound fuzzy propositions (FP). An atomic fuzzy proposition is a single statement

$$
\text { " } x \text { is } N \text { " }
$$

( $x$ is Negative), where $x$ is a linguistic variable and $N$ is a linguistic value of $x$. A compound fuzzy proposition is a composition of atomic fuzzy propositions using the connectives "And", "Or", and "Not", which represent fuzzy intersection, fuzzy union and respectively fuzzy complement.

An Example would be

$$
x \text { is } N \text { and } y \text { is } P \text {, }
$$

( $x$ is Negative and $y$ is Positive). Therefore, compound fuzzy propositions should be understood as fuzzy relations and one can see now how all the concepts above come into play:

- For connective "And”, use fuzzy intersections and any $t$-norm;

- For connective "Or", use fuzzy unions and any s-norm;

- For connective "Not", use fuzzy complement.

- A fuzzy IF-THEN rule is a conditional statement expressed as 


$$
\text { IF }<\text { Fuzzy Proposition>, THEN }<\text { Fuzzy Proposition }>\text {. }
$$

Because the fuzzy propositions are interpreted as fuzzy relations, the next question is how to interpret the IF-THEN operation. In classical propositional calculus, the expression IF $p$ THEN $q$ is written $p \rightarrow q$ with the implication arrow regarded as a normal connective and with $p$ and $q$ propositional variables whose values are either truth $(\mathrm{T})$ or false $(\mathrm{F})$. In that context, the classical logic operations "Not,- $\bar{p}$ ", "Or- $p \vee q$ " and "And- $p \wedge q$ ", respectively, are employed. Because the fuzzy IF-THEN rules can be viewed as replacing the $p$ and $q$ with fuzzy propositions, we can interpret the fuzzy IF-THEN rules by replacing the classical logic operators mentioned above. with fuzzy complement, fuzzy union and fuzzy intersection, respectively.

As an Example, some IF-THEN rules can have the form:

- "IF the service is Good, THEN the tip is Big".

- "IF the error is Positive Small, THEN the control input is Negative Big".

\section{A.3.1 The Compositional Rule of Inference}

The compositional rule of inference is a generalization of the following procedure: suppose we have a curve $y=f(x)$ from $x \in U$ to $y \in V$ and are given $x=a$, then from $x=a$ and $y$ $=f(x)$ we can infer that $y=b=f(a)$. The generalization goes one step further: given a fuzzy set $A$ ' representing the premise " $x$ is $A$ " and the fuzzy relation $Q=A \rightarrow B$ in $U \times V$ representing the premise "IF $x$ is $A$ THEN $y$ is $B$ ", a fuzzy set $B$ ' in $V$ representing the conclusion " $y$ is $B$ " " is inferred as

$$
\left.\mu_{B^{\prime}}(y)=\sup _{x \in U} t \mid \mu_{A^{\prime}}(x) \otimes \mu_{Q}(x, y)\right],
$$

where the symbol " $\otimes$ " denotes the $t$-norm operator.

The equality above can also be written in the form known as the Generalized Modus Ponens,

$$
\mu_{B^{\prime}}(y)=\sup _{x \in U} t\left[\mu_{A^{\prime}}(x), \mu_{A \rightarrow B}(x, y)\right] .
$$


Since there are a wide variety of fuzzy complement, fuzzy union and fuzzy intersection operators, a number of different interpretations of the fuzzy IF-THEN rules were proposed in the literature. In what concerns the work in this thesis, Mamdani Implications are of relevance, being also the most widely used implications in fuzzy logic control. Other types of implications, e.g., Zadeh, Gödel, etc., can be readily found in the literature, [35], [42].

Mamdani Implications: The fuzzy IF-THEN rule "IF $<\mathrm{FP}_{1}>$, THEN $<\mathrm{FP}_{2}>$ " is interpreted as a fuzzy relation $Q_{M M}$ or $Q_{M P}$ in $X \times Y$ with the membership function

$$
\mu_{Q_{M M}}(x, y)=\min \left[\mu_{F P_{1}}(x), \mu_{F P_{2}}(y)\right]
$$

or

$$
\mu_{Q_{M P}}(x, y)=\mu_{F P_{1}}(x) \cdot \mu_{F P_{2}}(y) .
$$

\section{A.4 Fuzzy Rule Base}

A Fuzzy Rule Base consists of a set of fuzzy IF-THEN rules. Specifically, the fuzzy rule base comprises the following fuzzy IF-THEN rules:

$$
R u^{(l)}: \quad \text { IF } x_{l} \text { is } A_{1}^{l} \text { and } \ldots \text { and } x_{n} \text { is } A_{n}^{l} \text {, THEN } y \text { is } B^{l}
$$

where $A_{i}^{l}$ and $B^{l}$ are fuzzy sets in $U_{i} \subset \mathbf{R}$ and $\mathrm{V} \subset \mathbf{R}$, respectively and $\mathbf{x}=\left[\begin{array}{llll}x_{1} & x_{2} & \ldots & x_{n}\end{array}\right]^{\mathrm{T}} \in U$ and $y \in V$ are the input and output (linguistic) variables of the fuzzy system, with $M$ the number of rules in the fuzzy rule base, $l=1,2, \ldots, M$. It can be seen that the above rule base has a canonical, general, form, since it includes most other rules types.

Definition A.4-1: A fuzzy set of fuzzy IF-THEN rules is complete if $(\forall) \mathbf{x} \in U,(\exists)$ at least one rule in the fuzzy rule base, $R u^{(l)}$, in the form of (A-14), such that $\mu A_{i}^{l}\left(x_{i}\right) \neq 0,(\forall) i=1$, $2, \ldots, n$. Intuitively, the completeness of a set of rules means that at any point in the input space, the membership value of the IF part of the rule is non-zero. 
Example: $\quad$ Consider a 2-input, 1-output fuzzy system with $U=U_{1} \times U_{2}=\left[\begin{array}{ll}0 & 1\end{array}\right] \times\left[\begin{array}{ll}0 & 1\end{array}\right]$ and $V=\left[\begin{array}{ll}0 & 1\end{array}\right]$. Define a three fuzzy sets $N_{1}, Z_{1}$ and $P_{1}$ in $U_{1}, N_{2}, Z_{2}$ and $P_{2}$ in $U_{2}$ and NB, NS, Z, $\mathrm{PS}, \mathrm{PB}$ in $V$, similar to the ones defined in Figure A-2. Assume that our input variables are the control error, $e \in U_{1}$, and its derivative, $\dot{e} \in U_{2}$ and the output is the control input to the plant, $u$, after the fuzzy controller processing $e$ and $\dot{e}$. Consider the following, complete set of fuzzy IFTHEN rules:

$R u^{(1)}: \quad$ IF $e$ is $N_{l}$ and $\dot{e}$ is $N_{2}$, THEN $y$ is $P B$

$R u^{(2)}: \quad$ IF $e$ is $N_{l}$ and $\dot{e}$ is $Z_{2}$, THEN $y$ is $P S$

$R u^{(3)}: \quad$ IF $e$ is $N_{l}$ and $\dot{e}$ is $P_{2}$, THEN $y$ is $Z$

$R u^{(4)}: \quad$ IF $e$ is $Z_{1}$ and $\dot{e}$ is $N_{2}$, THEN $y$ is $Z$

$R u^{(5)}$ : IF $e$ is $Z_{1}$ and $\dot{e}$ is $Z_{2}$, THEN $y$ is $Z$

$R u^{(6)}: \quad$ IF $e$ is $Z_{1}$ and $\dot{e}$ is $P_{2}$, THEN $y$ is $Z$

$R u^{(7)}: \quad$ IF $e$ is $P_{1}$ and $\dot{e}$ is $N_{2}$, THEN $y$ is $Z$

$R u^{(8)}: \quad$ IF $e$ is $P_{1}$ and $\dot{e}$ is $Z_{2}$, THEN $y$ is $N S$

$R u^{(9)}: \quad$ IF $e$ is $P_{1}$ and $\dot{e}$ is $P_{2}$, THEN $y$ is $N B$

They are graphically depicted in Figure A-3.
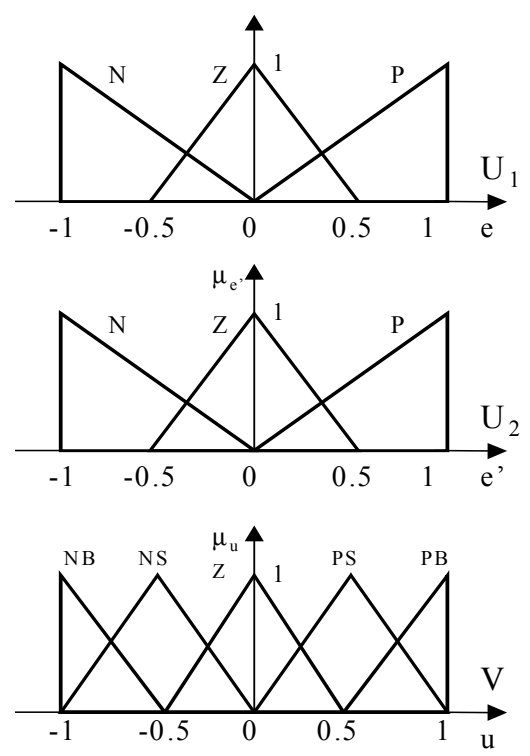

\begin{tabular}{|c|c|c|c|}
\hline $\mathrm{e}^{\mathrm{e}}$ & $\mathrm{N}$ & $\mathrm{Z}$ & $\mathrm{P}$ \\
\hline $\mathrm{N}$ & $\mathrm{PB}$ & $\mathrm{PS}$ & $\mathrm{Z}$ \\
\hline $\mathrm{Z}$ & $\mathrm{Z}$ & $\mathrm{Z}$ & $\mathrm{Z}$ \\
\hline $\mathrm{P}$ & $\mathrm{Z}$ & $\mathrm{NS}$ & $\mathrm{NB}$ \\
\hline
\end{tabular}

Figure A-3 - Membership Functions and Table for the Fuzzy IF-THEN Rule Base in (A-18) 
We can see that the numbers of rules in a complete fuzzy rule base increases exponentially with the dimension of the input space; this problem is called the curse of dimensionality.

\section{A.5 Fuzzy Inference Engine}

In a fuzzy inference engine, fuzzy logic principles are used to combine the fuzzy IFTHEN rules in the fuzzy rule base into a mapping from a fuzzy set $A$ in $U$ to a fuzzy set $B$ in $V$. There are two ways to infer with a set of rules: composition based inference and individual-rule based inference.

\section{A.5.1 Composition Based Inference}

In a composition - rule based inference, all rules in the fuzzy rule base are combined into a single fuzzy relation in $U \times V$, which is then viewed as a single fuzzy IF-THEN rule. There are two opposite set of arguments for what a set of rules should mean. The first one views the rules as independent conditional statements. If we accept this point of view, then a reasonable operator for combining the rules is union; this combination is called the Mamdani combination. The second one views the rules as strongly coupled conditional statements. If we accept this point of view, then we should use the operator intersection; this combination is called the Gödel combination.

Let $R u^{(l)}$ be a fuzzy relation, $R u^{(l)}=A_{1}^{l} \times \ldots \times A_{n}^{l} \rightarrow B^{l}$. From (A-8) we know that $A_{1}^{l} \times \ldots \times A_{n}^{l}$ is a fuzzy relation in $U=U_{1} \times \ldots \times U_{n}$ defined by

$$
\mu_{A_{1}^{l} \times \ldots \times A_{n}^{l}}\left(x_{1}, \ldots, x_{n}\right)=\mu_{A_{1}^{l}}\left(x_{1}\right) \otimes \ldots \otimes \mu_{A_{n}^{l}}\left(x_{n}\right)
$$

where $\otimes$ represents any $t$-norm operator, e.g. the intersection, (A-5). The implication " $\rightarrow$ " is defined according to various implication formulas, e.g. (A-13), (A-14). If we accept the first set of rules, then the set of $M$ rules in (A-17) are interpreted as a single fuzzy relation $Q_{M}$ in $U \times V$, defined by 


$$
Q_{M}=\bigcup_{l=1}^{M} R u^{(l)}
$$

This combination is called the Mamdani combination, where the union operator, $\cup$, is any $s$ norm. For the second view of a set of rules, the set of $M$ rules in (A-17) are interpreted as a single fuzzy relation $Q_{G}$ in $U \times V$, defined by

$$
Q_{G}=\bigcap_{l=1}^{M} R u^{(l)},
$$

which is called the Gödel combination, with the intersection operator, $\cap$, being any $t$-norm. $Q_{M}$ and $Q_{G}$ are thus viewed as a single fuzzy IF-THEN rule, given by the generalized modus ponens (A-14).

The composition based inference engine is less used in control, mainly due to computational efficiency and special properties, which can also be linked to efficiency, in some of their aspects.

\section{A.5.2 Individual-Rule Based Inference.}

In an individual - rule based inference, each fuzzy rule determines an output fuzzy set and the output of the whole fuzzy inference engine is the combination of the $M$ individual fuzzy sets. The combination can be taken either by union of by intersection.

The computational procedure of the individual-rule based inference (the composition based inference procedure is similar) is summarized as follows:

1. For the $M$ fuzzy IF-THEN rules in the form (A-17), determine the membership functions $\mu_{A_{1}^{\prime} \times \ldots \times A_{n}^{l}}\left(x_{1}, \ldots, x_{n}\right), l=1,2, \ldots, M$, according to (A-19).

2. View $A_{1}^{l} \times \ldots \times A_{n}^{l}$ as the $F P_{1}$ and $B^{l}$ as the $F P_{2}$ in the implications (A-15), (A-16) and determine $\mu_{R u^{(l)}}\left(x_{1}, \ldots, x_{n}, y\right)=\mu_{A_{1}^{l} \times \ldots \times A_{n}^{l} \rightarrow B}\left(x_{1}, \ldots, x_{n}, y\right), l=1,2, \ldots, M$, according to any one of these implications (or other implications used in the literature). 
3. For a given input fuzzy set $A^{\prime}$ in $U$, compute the output fuzzy set $B_{l}{ }^{\prime}$ in $V$ for each individual rule $R u^{(l)}$ according to the generalized modus ponens (A-14),

$$
\left.\mu_{B_{l}^{\prime}}(y)=\sup _{\mathbf{x} \in U} t \mid \mu_{A^{\prime}}(\mathbf{x}) \otimes \mu_{R u^{(l)}}(\mathbf{x}, y)\right] .
$$

4. The output of the fuzzy inference engine is the combination of the $M$ fuzzy sets $\left\{B_{l}, \ldots, B_{M}\right\}$ either by union, i.e.,

$$
\mu_{B^{\prime}}(y)=\mu_{B_{1}^{\prime}}(y) \oplus \ldots \oplus \mu_{B_{M}^{\prime}}(y),
$$

or by intersection, i.e.,

$$
\mu_{B^{\prime}}(y)=\mu_{B_{1}^{\prime}}(y) \otimes \ldots \otimes \mu_{B_{M}^{\prime}}(y),
$$

where $\oplus$ and $\otimes$ denote the $s$-norm and $t$-norm operators, respectively.

There are a number of fuzzy inference engines that are commonly used in fuzzy systems and control. The most used one in fuzzy control, employed also in this study is the product inference engine.

Product Inference Engine: In the Product Inference Engine, we use: (i) individual rule based inference with union combination, (A-23), (ii) Mamdani's product implication (A-16) and (iii) algebraic product for all the $t$-norm operators and $\max$ for all $s$-norm operators. Specifically, from (A-22), (A-23), (A-16) and (A-19), we obtain the product inference engine as

$$
\mu_{B^{\prime}}(y)=\max _{l=1}^{M}\left[\sup _{\mathbf{x} \in U}\left(\mu_{A^{\prime}}(\mathbf{x}) \cdot \prod_{i=1}^{n} \mu_{A_{i}^{l^{\prime}}}\left(x_{i}\right) \cdot \mu_{B^{\prime}}(y)\right)\right]
$$

Equation (A-25) gives the fuzzy set $B$ ' in $V$, given the fuzzy set $A^{\prime}$ ' in $U$, according to this engine. Another Inference engine that is widely used is the minimum inference engine.

Minimum Inference Engine: In the Minimum Inference Engine, we use: (i) individual rule based inference with union combination, (A-23), (ii) Mamdani's minimum implication (A- 
15 ) and (iii) $\min$ for all the $t$-norm operators and $\max$ for all $s$-norm operators. Specifically, from (A-22), (A-23), (A-15) and (A-19), we obtain the product inference engine as

$$
\mu_{B^{\prime}}(y)=\max _{l=1}^{M}\left[\sup _{\mathbf{x} \in U} \min \left(\mu_{A^{\prime}}(\mathbf{x}), \mu_{A_{1}^{l}}\left(x_{1}\right), \ldots, \mu_{A_{n}^{l}}\left(x_{n}\right), \mu_{B^{l}}(y)\right)\right]
$$

The main advantage of the above engines is their computational simplicity; this is especially true for the product inference engine. Also, they are intuitively appealing for many practical problems and it can be shown that although the individual-rule based and composition based inferences are conceptually different, they produce the same fuzzy inference engine in certain important cases.

It can be easily be shown, by direct substitution, that if the fuzzy set $A^{\prime}$ is a fuzzy singleton, that is,

$$
\mu_{A^{\prime}}(\mathbf{x})=\left\{\begin{array}{lc}
1 & \text { if } \mathbf{x}=\mathbf{x}^{*} \\
0 & \text { otherwise }
\end{array}\right.
$$

where $\mathbf{x}^{*}$ is some point in $U$, then the product inference engine is simplified to

$$
\mu_{B^{\prime}}(y)=\max _{l=1}^{M}\left(\prod_{i=1}^{n} \mu_{A_{i}^{\prime}}\left(x_{i}^{*}\right) \cdot \mu_{B^{l}}(y)\right)
$$

and the minimum inference engine is simplified to

$$
\mu_{B^{\prime}}(y)=\max _{l=1}^{M}\left[\min \left(\mu_{A_{1}^{l}}\left(x_{1}\right), \ldots, \mu_{A_{n}^{l}}\left(x_{n}\right), \mu_{B^{l}}(y)\right)\right] .
$$

A disadvantage of the product and minimum inference engines is that if at some points $\mathbf{x} \in U$ their membership functions are very small, then the output of the inference engine will also be very small, which sometimes causes problems in implementation. There are other fuzzy inference engines that overcome this disadvantage, e.g. Lukasiewicz, Zadeh, Dienes-Rescher, [36], but they are not of interest here, as we do not exhibit the specific problem mentioned above. 


\section{A.6 Fuzzifiers and Defuzzifiers}

Fuzzifiers and Defuzzifiers are the interfaces between the fuzzy inference engine and the real numbers environment.

\section{A.6.1 Fuzzifiers}

A fuzzifier is defined as a mapping from a real valued point $\mathbf{x}^{*} \in U \subset \mathbf{R}^{\mathrm{n}}$ to a fuzzy set $A$, in $U$.

There are several criteria in designing the fuzzifier. First, the fuzzy set $A$ ' should have the largest membership value at $\mathbf{x}^{*}$. Second, if the input to the fuzzy system is corrupted by noise, then it is desirable that the fuzzifier should help to suppress the noise. Third, the fuzzifier should offer simple computations. There are three major fuzzifiers:

a) Singleton Fuzzifier: The singleton fuzzifier maps a real-valued point $\mathbf{x}^{*} \in U$ into a fuzzy singleton $A$ ' in $U$, which has membership value 1 at $\mathbf{x}^{*}$ and 0 at all other points in $\mathrm{U}$; that is,

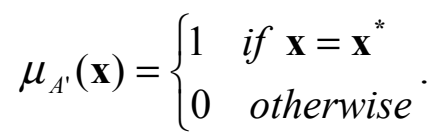

b) Triangular Fuzzifier: The triangular fuzzifier maps a real-valued point $\mathbf{x}^{*} \in U$ into a fuzzy singleton $A^{\prime}$ in $\mathrm{U}$, which has membership function

$$
\mu_{A^{\prime}}(\mathbf{x})=\left\{\begin{array}{l}
\left(1-\frac{\left|x_{1}-x_{1}^{*}\right|}{b_{1}}\right) \otimes \ldots \otimes\left(1-\frac{\left|x_{n}-x_{n}^{*}\right|}{b_{n}}\right) \text { if }\left|x_{i}-x_{i}^{*}\right| \leq b_{i}, i=1,2, \ldots, n . \\
0 \text { otherwise }
\end{array}\right.
$$

where $b_{i}$ are positive parameters and the $t$-norm $\otimes$ is usually chosen as algebraic product or $\min$.

c) Gaussian Fuzzifier: The Gaussian fuzzifier maps a real-valued point $\mathbf{x}^{*} \in U$ into a fuzzy singleton $A$ ' in $\mathrm{U}$, which has membership function 


$$
\mu_{A^{\prime}}(\mathbf{x})=e^{-\left(\frac{x_{1}-x_{1}^{*}}{a_{1}}\right)^{2}} \otimes \ldots \otimes e^{-\left(\frac{x_{n}-x_{n}^{*}}{a_{n}}\right)^{2}} .
$$

where $\mathrm{a}_{i}$ are positive parameters and the $t$-norm $\otimes$ is usually chosen as algebraic product or min.

The Singleton Fuzzifier is one of the most often used in control applications. However, one can see, even intuitively, that the Triangular and Guaussian fuzzifiers offer training capabilities through their $b_{i}$ and $a_{i}$, parameters, both on- and off-line, as well as potential noise filtering capabilities.

\section{A.6.2 Defuzzifiers}

The defuzzifier is defined as a mapping from the fuzzy set $B^{\prime}$ in $V \subset \mathbf{R}$, which is the output of the fuzzy inference engine, to a crisp point $y^{*} \in U$. Conceptually, the task of the defuzzifier is to specify a point in $\mathrm{V}$ that best represents the fuzzy set $B^{\prime}$ (similar to the mean value of a random variable). Three main defuzzifiers are introduced.

a) Center of Gravity Defuzzifier: The Center of Gravity (CG) defuzzifier specifies the $y^{*}$ as the center of the area covered by the membership function of $B^{\prime}$, i.e.,

$$
y^{*}=\frac{\int_{V} y \cdot \mu_{B^{\prime}}(y) d y}{\int_{V} \mu_{B^{\prime}}(y) d y} .
$$

Figure A-4 shows this operation graphically. 


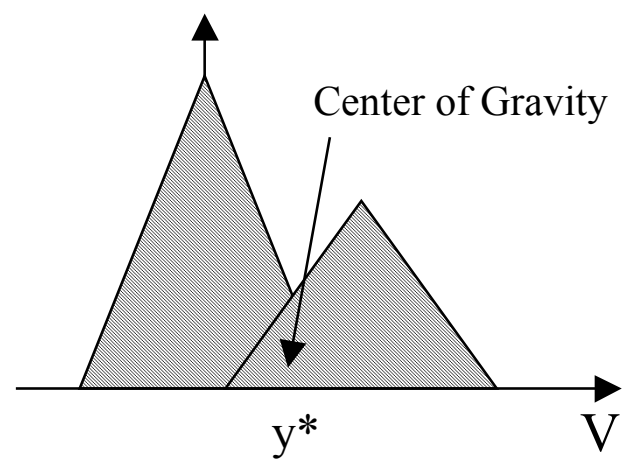

Figure A-4 - Center of Gravity Defuzzifier

If $\mu_{B} \cdot(y)$ is viewed as the probability density function of a random variable, then the center of gravity defuzzifier gives the mean value of the random variable. The advantage of the center of gravity defuzzifier lies in its intuitive plausibility, but the disadvantage is that it is computationally intensive since the membership function $\mu_{B} \cdot(y)$ is usually irregular and therefore the integrations are difficult to compute. The next defuzzifier tries to overcome this by approximating (A-33) with a simpler formula.

b) Center Average Defuzzifier: Because the fuzzy set $B^{\prime}$ is the union or intersection of $M$ fuzzy sets, a good approximation of (A-33) is the weighted average of the centers of the $M$ fuzzy sets, with the weights equal with the heights of the corresponding fuzzy sets. Specifically, let $y^{l}$ be the center of the $l^{\text {th }}$ fuzzy set and $w_{l}$ be its height. The Center Average Defuzzifier determines $y^{*}$ as

$$
y^{*}=\frac{\sum_{l=1}^{M} y^{l} \cdot w_{l}}{\sum_{l=1}^{M} w_{l}}
$$

Figure A-5 illustrates this operation graphically, for $M=2$. 


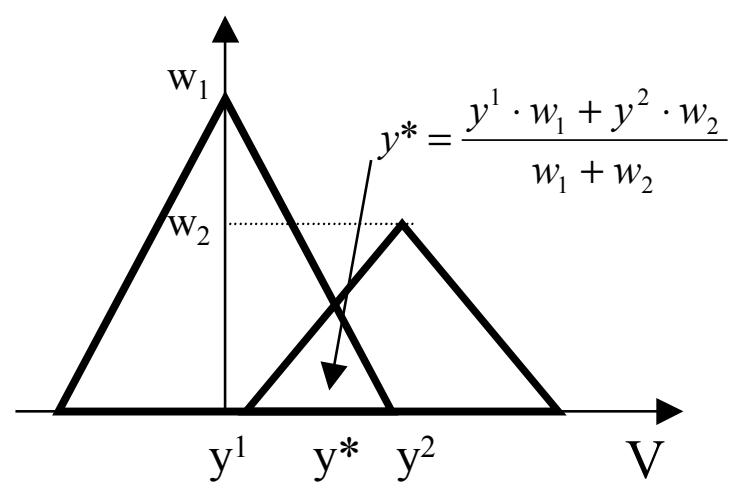

Figure A-5 - Center Average Defuzzifier

The center average defuzzifier is the most commonly used defuzzifier in fuzzy systems control. It is computationally simple and intuitively appealing; also, small changes in $y^{l}$ and $w_{l}$ result in small changes in $y^{*}$.

c) Maximum Defuzzifier: Conceptually, the maximum defuzzifier chooses the $y^{*}$ as the point in $V$ at which $\mu_{B} \cdot(y)$ achieves its maximum value. If we define the set

$$
\operatorname{hgt}\left(B^{\prime}\right)=\left\{y \in V \mid \mu_{B^{\prime}}(y)=\sup _{y \in V} \mu_{B^{l}}(y)\right\}
$$

the maximum defuzzyfier defines $y^{*}$ as an arbitrary element in $\operatorname{hgt}\left(B^{\prime}\right)$. If $\operatorname{hgt}\left(B^{\prime}\right)$ contains more than one point, then we may still use (A-35) or use the smallest of maxima,

$$
y^{*}=\inf \left\{y \in \operatorname{hgt}\left(B^{\prime}\right)\right\}
$$

largest of maxima,

$$
y^{*}=\sup \left\{y \in \operatorname{hgt}\left(B^{\prime}\right)\right\}
$$

or mean of maxima defuzzifiers, which is defined similarly to the center of gravity defuzzifier. Maximum defuzzifiers are intuitively plausible and computationally simple, but in some cases small changes in $B$ ' may result in large changes in $y^{*}$. 
d) Singleton Defuzzifier: One particular case, employed a lot in fuzzy logic control for $\mathrm{DC} / \mathrm{DC}$ converters and used in this thesis, is to consider a similar defuzzifier to the fuzzifier in (A-30) above. That is, $y^{*}$ is selected out of each, triangular for example, membership function curve for the fuzzy subsets in $V$ as the center of that respective subset/membership function. For example, in Figure A-5, the "singleton defuzzifiers would be $y^{l}$ and $y^{2}$.

A complete example of how one employs the above concepts to built a fuzzy logic controller is given in the next paragraph.

\section{A.7 Fuzzy Systems as Nonlinear Mappings - Fuzzy Controller Structure}

\section{A.7.1 Fuzzy Systems with Center Average Defuzzifier}

One of the most used fuzzy systems are the fuzzy systems with center average defuzzifier. We have the following lemma.

Lemma A.7-1: Suppose that the fuzzy set $B^{l}$ in (A-17) is normal (unity height) with center $y^{l}$. Then the fuzzy systems with fuzzy rule base (A-17), product inference engine (A-25), singleton fuzzifier (A-30) and center average defuzzifier (A-34) are of the following form:

$$
f(x)=\frac{\sum_{l=1}^{M} y^{l} \cdot\left(\prod_{i=1}^{n} \mu_{A_{i}^{l}}\left(x_{i}\right)\right)}{\sum_{l=1}^{M}\left(\prod_{i=1}^{n} \mu_{A_{i}^{l}}\left(x_{i}\right)\right)}
$$

where $\mathrm{x} \in U \subset \mathbf{R}^{\mathrm{n}}$ is the input to the fuzzy system and $f(\mathrm{x}) \in V \subset \mathbf{R}^{\mathrm{n}}$ is the output of the fuzzy system.

Proof: Substituting (A-30) into (A-25), we have

$$
\mu_{B^{\prime}}(y)=\max _{l=1}^{M}\left(\prod_{i=1}^{n} \mu_{A_{i}^{l}}\left(x_{i}^{*}\right) \cdot \mu_{B^{l}}(y)\right)=\max _{l=1}^{M}\left(\prod_{i=1}^{n} \mu_{A_{i}^{l}}\left(x_{i}^{*}\right)\right)
$$


since $\mu_{B^{l}}(y)=1, B^{l}$ being normal. Additionally, because for a given input $x_{i}{ }^{*}$, the center of the l'th fuzzy set in (A-39), i.e. the fuzzy set with the membership function $\mu_{A_{i}^{l}}\left(x_{i}^{*}\right) \cdot \mu_{B^{l}}(y)$ is the center of $B^{l}$, we see that $y^{l}$ in (A-34) is the same $y^{l}$ in (A-38). Hence, using the center average defuzzifier for (A-39), we obtain

$$
y^{*}=\frac{\sum_{l=1}^{M} y^{l} \cdot\left(\prod_{i=1}^{n} \mu_{A_{i}^{l}}\left(x_{i}^{*}\right)\right)}{\sum_{l=1}^{M}\left(\prod_{i=1}^{n} \mu_{A_{i}^{l}}\left(x_{i}^{*}\right)\right)}
$$

Using the notations in (A-38), we have that $x^{*}=x$ and $y^{*}=f(x)$, q.e.d..

From Lemma A.7-1 we see that the fuzzy system is a nonlinear mapping from $\mathrm{x} \in U \subset \mathbf{R}^{\mathrm{n}}$ to $f(\mathrm{x}) \in V \subset \mathbf{R}^{\mathrm{n}}$, where the detailed formula for this mapping is given by (A-38). These fuzzy systems are the most commonly fuzzy systems used in the literature; they are computationally simple and intuitively appealing. From (A-38) we see that the output of the fuzzy system is a weighted average of the centers of the fuzzy sets in the THEN parts of the rules, where the weights equal the membership values of the fuzzy sets in the IF part of the rule. Hence, the more the input point agrees with the IF part of the rule, the larger weight this rule is given, which makes sense, intuitively. By choosing different forms of membership functions, e.g., Gaussian, and/or different inference engines, we can obtain different classes of fuzzy systems. These systems can then offer both on- and off-line training, together with certain noise rejection capabilities.

Lemma A2.7-1 reveals also the dual role of fuzzy systems. On one hand, fuzzy systems are rule based systems that are constructed from a collection of linguistic rules. On the other hand, fuzzy systems are nonlinear mappings that in many cases can be represented by precise and compact formulas such as (A-38). Because nonlinear mappings are often easy to implement, fuzzy systems have found their way into a variety of engineering applications, as in this study.

As known, in most forms fuzzy systems are universal approximators, [36]. Specifically, for any kind of nonlinear operations the problem may require, it is always possible to design a fuzzy system that performs the required operation with any degree of accuracy. However, the theorems in the literature only give an existence result. In practice, one must develop approaches 
that can find good fuzzy systems for the particular application considered based on the knowledge about the plant and its control action, as also shown also this study.

Example: The structure of a fuzzy logic controller can be depicted in Figure A-6, below, showing, generically, the controller used in this thesis, for the new current sharing compensator.

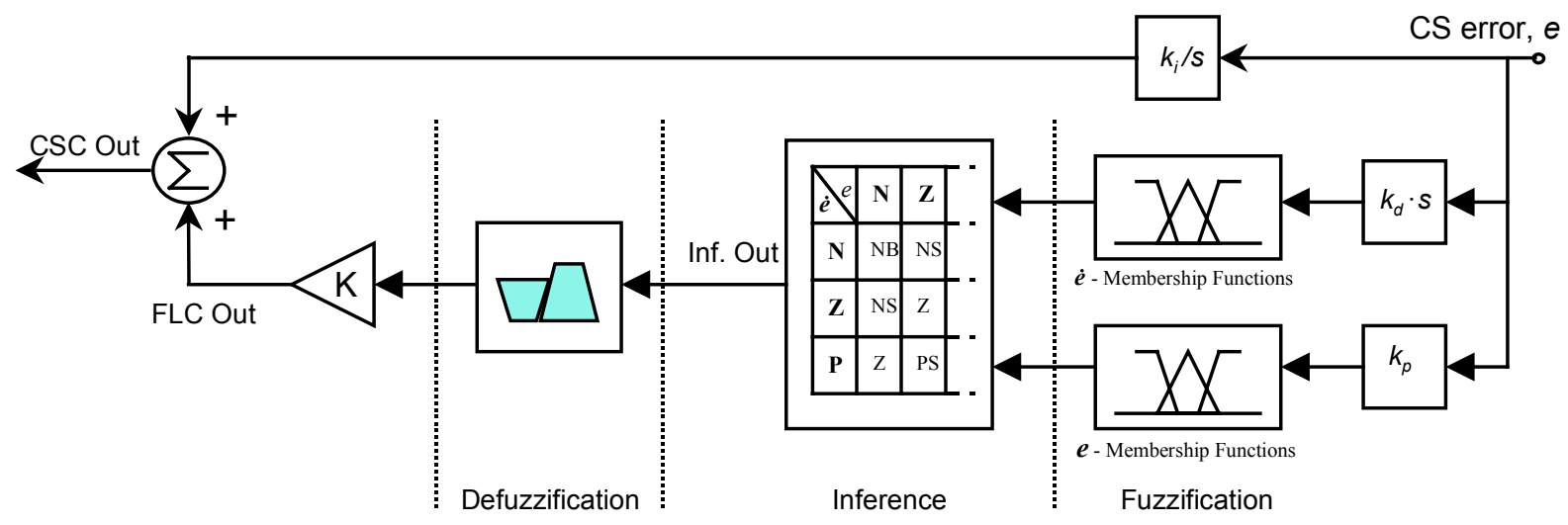

Figure A-6 - Fuzzy Logic Controller used for the MS CSC in the thesis

The input vector $x$ has two components, the CS error, $e$ and its derivative, $\dot{e}$. A three range partition, negative $(\mathrm{N})$, zero $(\mathrm{Z})$ and positive $(\mathrm{P})$, is chosen for the inputs, $e$ and $\dot{e}$. A more refined five-range partition, negative-big (NB), negative-small (NS), zero (Z), positive-small (PS) and positive-big (PB) is used for the consequents, using singleton fuzzifiers. As shown above, we employed a product inference engine and center average defuzzifier. Based on the usual action of a PD cell, by looking at its output range as a function of the input range from (2), a rule-based inference is chosen for each consequent value, following the methodology in Paragraph 5.1.

The FLC in equation (A-40), also employed in this study, can be generalized and put in a vector form. For example, equation (A-18) considered two components, $e$ and $\dot{e}$ for the input vector e. Denoting this vector with $x$ as before, assuming it has $n$ components and considering the output $y$, one obtains (A-40), with $M$ the number of IF - THEN rules. If now we collect the consequent singletons, $y^{l}, l=1,2, \ldots, M$, in a vector $\theta$ and the input membership functions combination in a vector $\boldsymbol{\xi}(\mathbf{x})$, having its $l^{\text {th }}$ element equal with 


$$
\xi^{l}=\frac{\prod_{i=1}^{n} \mu_{A_{i}^{l}}\left(x_{i}\right)}{\sum_{l=1}^{M}\left(\prod_{i=1}^{n} \mu_{A_{i}^{l}}\left(x_{i}\right)\right)}
$$

we can write the fuzzy control output as

$$
y=\boldsymbol{\theta}^{\mathrm{T}} \cdot \xi(\mathbf{x})
$$

or, in the context of the output being the control quantity and the input being the control error,

$$
u=\boldsymbol{\theta}^{\mathrm{T}} \cdot \xi(\mathbf{e})
$$

This form has been employed in the proof of existence for a Lyapunov stable trajectory for such an FLC, Section 5.2, Eqs. (5.2-4) - (5.2-7) and, as a concept, throughout this thesis. 


\section{Appendix B}

\section{BASIC ELEMENTS of LYAPUNOV STABILITY}

This approach to system's stability is named after the russian scientist A. M. Lyapunov, 1857 - 1918, who was a school friend of Markov and later a student of Chebyshev. He did important work on differential equations, potential theory, stability of systems and probability theory. His work concentrated on the stability of equilibrium and motion of a mechanical system and the stability of a uniformly rotating fluid. Lyapunov's methods, introduced by him in 1899, provide ways of determining the stability of sets of ordinary differential equations.

The Lyapunov functions can be considered as a generalization of the concept of distance or energy. If the "distance" of the state to an equilibrium point along any trajectory of $\dot{\mathbf{x}}=\mathbf{A} \cdot \mathbf{x}$ decreases with time, then $\mathbf{x}(t)$ must tend to $\mathbf{0}$ as $t \rightarrow \infty$, , [13] - [15], [36] - [38], [43], [44]. The following definition of a system stable In the Sense Of Lyapunov (i.s.o.L.), [43], can thus be given.

Definition B.1: An equilibrium point, $x_{e}$, of the system $\dot{\mathbf{x}}=\mathbf{A}(t) \cdot \mathbf{x}$ is stable i.s.o.L., or, for the discussion here, simply stable, if for any $\varepsilon>0,(\exists)$ a value $\delta\left(t_{0}, \varepsilon\right)>0$ such that if $\left\|\boldsymbol{x}\left(t_{0}\right)-\boldsymbol{x}_{\boldsymbol{e}}\right\|<$ $\delta$, then $\left\|\boldsymbol{x}(t)-\boldsymbol{x}_{\boldsymbol{e}}\right\|<\varepsilon,(\forall) t>t_{0}$.

- The equilibrium point is uniformly stable i.s.o.L. if $\delta=\delta\left(t_{0}, \varepsilon\right)$, i.e., the constant $\delta$ does not depend on the initial time $t_{0}$.

- The equilibrium point is asymptotically stable if the state vector tends toward zero, i.e., if $\left\|\boldsymbol{x}(t)-\boldsymbol{x}_{\boldsymbol{e}}\right\| \rightarrow 0$ as $t \rightarrow \infty$. In addition, the equilibrium point is said to be exponentially stable if we can find constants $\gamma>0$ and $\lambda>0$ such that for all $t>t_{0},\left\|\boldsymbol{x}(t)-\boldsymbol{x}_{\boldsymbol{e}}\right\| \leq \gamma \cdot \mathrm{e}^{\lambda \cdot(t-t o)}\left\|\boldsymbol{x}\left(t_{0}\right)-\boldsymbol{x}_{\boldsymbol{e}}\right\|$.

A Linear Time Invariant (LTI) system that is asymptotically stable is also exponentially stable since all solutions of LTI systems that approach the origin do so by exponential decay. This is not the case for time varying systems and not always the case for nonlinear systems. 
One can also speak of systems that are stable locally or stable globally (or in the large). In the case of globally stable systems, the constant $\delta$ can be chosen arbitrarily large. This implies that if an equilibrium point is asymptotically stable, then the trajectory from any initial condition will asymptotically approach it. This, in turn, implies that there can be only one equilibrium point that is asymptotically stable in the large.

DC-DC converters are time invariant systems, which is the case for all applications throughout this study. The following theorem describes the Lyapunov stability for this type of systems.

Theorem B.1: $\quad$ The equilibrium of the LTI system $\dot{\mathbf{x}}=\mathbf{A} \cdot \mathbf{x}$ is stable i.s.o.L. if and only if all the eigenvalues of $\mathbf{A}$ have non-positive real parts, and those that have zero real parts are nonrepeated. The equilibrium of the LTI system $\dot{\mathbf{x}}=\mathbf{A} \cdot \mathbf{x}$ is stable i.s.o.L. if and only if all the eigenvalues of $\mathbf{A}$ have negative real parts.

The last two results are commonly used to test for Lyapunov stability. They are directly extensible to discrete systems if we replace all the references to the left half plane with references to the inside of the unit circle. A different method for testing the stability of linear, zero-input systems is called Lyapunov's direct method, [43], [44]. It is based on the concept of energy and dissipative systems. The physically intuitive explanation is that an isolated system will have a certain amount of a non-negative, abstract, quantity that is similar to energy. If its energy is always decreasing, we think of it as being stable and if its energy increases, we think of it as being unstable. However, because not all state space systems are descriptions of physical phenomena normally endowed with real energy, we generalize the concept; this is the basis of Lyapunov's direct method.

Theorem B.2: $\quad$ The origin of the LTI system $\dot{\mathbf{x}}(t)=\mathbf{f}(\mathbf{x}(t))$ is Lyapunov stable if there exists a positive definite function $V(\boldsymbol{x})$ such that its derivative

$$
\dot{V}(\mathbf{x})=\left(\nabla_{\mathbf{x}} V(\mathbf{x})\right)^{T} \cdot \dot{\mathbf{x}}=\left(\nabla_{\mathbf{x}} V(\mathbf{x})\right)^{T} \cdot \mathbf{f}(\mathbf{x})
$$

is negative semi-definite. If $\dot{V}(\mathbf{x})$ is negative definite, the origin is asymptotically stable. 
Theorem B.2 holds for discrete systems if we replace the continuous time, $t$, with the discrete time $\operatorname{step}(\mathrm{s}), k, k+1$, etc. .

A function $V(\mathbf{x})$ satisfying these requirements is called a Lyapunov function. The Lyapunov function is not unique; rather, many Lyapunov functions may be found for a given system. Likewise, the inability to find a Lyapunov function does not mean that the system is unstable.

Even though it shows that stable or asymptotically stable systems do have Lyapunov functions, this theorem do not provide a mean to find them. For highly nonlinear systems, in particular, finding such a function can prove quite difficult. The usual procedure is to guess, using the application specific physical insight, at a Lyapunov function, called candidate Lyapunov function and then test to see if it satisfy the above conditions. However, for linear and bilinear systems, which is the case of the systems in this thesis, Lyapunov functions generally take quadratic forms, being related to the system's energy.

\section{B.1 Lyapunov Functions for LTI Systems}

In the case of linear and most bilinear systems, it is sufficient to consider quadratic forms as Lyapunov candidate functions. This is because the parabolic shape of a quadratic functions satisfies all the criteria of the above theorems.

Consider the LTI system

$$
\dot{\mathbf{x}}=\mathbf{A} \cdot \mathbf{x}
$$

and the candidate Lyapunov function

$$
V(\mathbf{x})=\mathbf{x}^{\mathbf{T}} \cdot \mathbf{P} \cdot \mathbf{x},
$$

where the matrix $\mathbf{P}$ is positive definite. Then, using the last two equalities, the time rate of change of this energy-like function is

$$
\dot{V}(\mathbf{x})=\dot{\mathbf{x}}^{T} \cdot \mathbf{P} \cdot \mathbf{x}+\mathbf{x}^{T} \cdot \mathbf{P} \cdot \dot{\mathbf{x}}=(\mathbf{A} \cdot \mathbf{x})^{T} \cdot \mathbf{P} \cdot \mathbf{x}+\mathbf{x}^{T} \cdot \mathbf{P} \cdot(\mathbf{A} \cdot \mathbf{x})=\mathbf{x}^{T} \cdot\left(\mathbf{A}^{T} \cdot \mathbf{P}+\mathbf{P} \cdot \mathbf{A}\right) \cdot \mathbf{x} \cdot(\mathrm{B}-4)
$$


If the system is asymptotically stable, for example, to satisfy theorem B.2, it is necessary that the matrix $\mathbf{A}^{\mathbf{T}} \cdot \mathbf{P}+\mathbf{P} \cdot \mathbf{A}$ be negative definite and this requires that the matrix equation

$$
\mathbf{A}^{T} \cdot \mathbf{P}+\mathbf{P} \cdot \mathbf{A}=-\mathbf{Q}
$$

be true for some positive definite matrix Q. Equation (B-5) is known as the Lyapunov equation.

The Lyapunov's direct method provides for the "sufficiency" part and not for the "necessity" one. It asserts that if a Lyapunov function is found, then the system is stable. It does not say that if a candidate Lyapunov function fails, the system is not stable. Therefore, if a positive definite matrix $\mathbf{P}$ is chosen and we compute a $\mathbf{Q}$ that is indefinite, nothing ca be concluded about the system's stability i.s.o.L. However, if $\mathbf{Q}$ results negative definite, instability can be shown. A more powerful result is obtained by working in the reverse direction, i.e., by starting with a positive definite $\mathbf{Q}$ matrix and using equation (B-5) to determine $\mathbf{P}$. It usually turns out that any symmetric positive definite $\mathbf{Q}$ will suffice and $\mathbf{Q}=\mathbf{I}$ is often used. One obtains the following theorem.

Theorem B.3: $\quad$ The origin of the system (B-2) is asymptotically stable if and only if, given a positive matrix $\mathbf{Q}$, the matrix equation (B-5) has a solution $\mathbf{P}$ that is positive definite.

The sufficiency proof is straight forward since if $\mathbf{P}$ and $\mathbf{Q}$ are both positive definite, Lyapunov's direct method has already shown that the system will be asymptotically stable, because $\mathbf{x}^{\mathbf{T}} \cdot \mathbf{P} \cdot \mathbf{x}$ will have been demonstrated to be a Lyapunov function.

The necessity proof can be readily found in the literature, e.g., [43] and it proves that if the origin is known to be a stable equilibrium and $\mathbf{Q}$ is positive definite, a unique, positive definite $\mathbf{P}$ is obtained as the result of

$$
\mathbf{P}=\int_{0}^{\infty} e^{A^{T} \cdot t} \cdot \mathbf{Q} \cdot e^{A \cdot t} d t
$$

In view of the one to one relationship between $\mathbf{P}$ and $\mathbf{Q}$ established by the Lyapunov equation, (B-5), one has to also note that this theorem does not directly extend to Lyapunov stability; that is, starting with an arbitrary positive definite $\mathbf{Q}$ will give a definite answer, however, starting with an arbitrary positive semi-definite $\mathbf{Q}$ or starting with an arbitrary positive definite $\mathbf{P}$ may not 
give a definite answer. The following, LaSalle's theorem, generalizes the claim for asymptotic stability when employing this method of finding Lyapunov functions.

Theorem B.4 (La Salle's theorem): Within the region of the state space for which the derivative

of the candidate Lyapunov function is such that $\dot{V}(\mathbf{x}) \leq 0$, let $Z$ denote the subset of the state space in which $\dot{V}(\mathbf{x})=0$. Within $Z$, let $M$ denote the largest invariant subset. Then, every initial state approaches $M$, even if $V$ is not positive-definite.

By an extension of La Salle's theorem above, [43] we can provide the following generalization:

Theorem B.4: $\quad$ The origin of the system (B-2) described by matrix $\mathbf{A}$ is asymptotically stable if and only if, given a positive semi-definite matrix $\mathbf{Q}$, such that $\mathbf{x}^{\mathbf{T}} \cdot \mathbf{Q} \cdot \mathbf{x}$ is not identically zero when evaluated on a non-zero trajectory of (B-2), the matrix Lyapunov equation (B-5) has a solution $\mathbf{P}$ that is positive definite.

\section{B.2 Use of Lyapunov's Direct Method in Feedback Design}

Stability is an overriding requirement in almost all control designs. It is possible to use the Lyapunov's methods in the majority of situations. Since $V(\mathbf{x}, t)$ does not depend on $\dot{\mathbf{x}}$ and hence on $u$ or $r$ - but $\dot{V}$ does - the following controller design procedure can be employed, as it is also the case in this thesis.

First, select a Lyapunov function for the homogeneous system equations, which should be positive definite. In our study, this was the case of the energy in the increment in the reactive elements currents/voltages giving the states of the DC/DC converters, as shown in Chapter 4. This ensures that the uncontrolled system is at least stable i.s.o.L. Then allow the control variable to be non-zero and calculate these control dependent terms to force a negative $\dot{V}(\mathbf{x})$, as much as possible within the available control limits. $V$ itself will not be affected, but we would obtain a stable control system which approximates a minimum time response, high control effort system, [44]. This typically leads to a nonlinear control law, even when the original system is linear. As a simple example, consider a system of the form 


$$
\dot{\mathbf{x}}=\mathbf{A} \cdot \mathbf{x}+\mathbf{B} \cdot \mathbf{u}
$$

with the candidate Lyapunov quadratic function given in (B-3). Assume that we determined a positive definite solution, $\mathbf{P}$, of the Lyapunov equation (B-5) by using $\mathbf{Q}=\mathbf{I}$ (one can also assume a symmetric form for $\mathbf{P}$, in the calculations). Calculating the derivative of the Lyapunov function we obtain thus

$$
\dot{V}(\mathbf{x})=-\mathbf{x}^{T} \cdot \mathbf{Q} \cdot \mathbf{x}+2 \cdot \mathbf{u}^{T} \cdot \mathbf{B} \cdot \mathbf{P} \cdot \mathbf{x}=-\mathbf{x}^{T} \cdot \mathbf{x}+2 \cdot \mathbf{u}^{T} \cdot \mathbf{B} \cdot \mathbf{P} \cdot \mathbf{x} .
$$

The control $\mathbf{u}(t)$ is selected to minimize $\mathbf{u}^{\mathbf{T}} \cdot \mathbf{B}^{\mathbf{T}} \cdot \mathbf{P} \cdot \mathbf{x}$ subject to the restriction placed upon $\mathbf{u}(t)$ in the specific application. Obviously, $\mathbf{u}(t)$ should be parallel to $\mathbf{B}^{\mathbf{T}} \cdot \mathbf{P} \cdot \mathbf{x}$ but with opposite sign and have its largest possible magnitude. That is,

$$
\mathbf{u}(t)=\frac{-\mathbf{B}^{\mathbf{T}} \cdot \mathbf{P} \cdot \mathbf{x}(t)}{\left\|\mathbf{B}^{\mathbf{T}} \cdot \mathbf{P} \cdot \mathbf{x}(t)\right\|} .
$$

This controller will rapidly drive the state back to zero if it is perturbed by initial conditions or disturbances. Other control system designs might require just being stable, with no stringent minimum time response, maximum control effort, just demonstrated. In either case, depending on their performance and feasibility, the newly obtained, Lyapunov control laws can be used either by themselves or in conjunction with an existing, more satisfactory control They can be employed either as an adaptive, stable trajectory or as a supervisory control, as it was pointed out in the thesis. 


\section{Vita}

Bogdan Tomescu was born in Bucharest, Romania, in 1963. He graduated his MSEE from Bucharest Polytechnic Institute, Electronics and Telecommunications Department, in 1987. From 1987 to 1990, he worked as an R\&D Engineer at the Bucharest Electrotechnics Research and Design Institute. In 1990 he joined the Bucharest Polytechnic Institute, Electronics and Telecommunications Department, working as an Assistant Professor at the Applied Electronics Chair - Power Electronics and pursuing his doctoral degree.

He immigrated to the United States at the beginning of the nineties and after holding several engineering positions in the electronics industry, he is with Space Systems/Loral, Palo Alto CA. He is presently finishing his $\mathrm{PhD}$ at VA Tech, Blacksburg VA, where he was a Graduate Research Assistant at VA Power Electronics Center and a Teaching Assistant as part of the Electrical Engineering Department. His interests include DC/DC converters modeling and control, emerging neuro-fuzzy techniques and electric propulsion systems.

Bogdan Tomescu 